\title{
Einige Wege und Ziele der Familienforschung, mit Rücksicht auf die Psychiatrie.
}

\author{
Von \\ Dr. E. Rïdin, Privatdozent, \\ Oberarzt der psychiatrischen Klinik München. \\ Mit 17 Textfiguren. \\ (Eingegangen am 25. September 1911.)
}

Ein Vorgang, der den Psychiater von jeher besonders interessierte, ist die Vererbung.

Die von vielen Seiten berechnete ,erbliche Belastung“ der Geisteskranken wurde immer als bedeutsam angenommen, und auch die neuesten Belastungsberechnungen von Koller und Diem z. B., die in kritischer Weise die Verhältnisse bei den geistig Kranken mit denen bei seelisch Gesunden verglichen, haben die alte allgemeine Auffassung im wesentlichen bestätigt, daß in der Verwandtschaft Geisteskranker psychische Anomalien häufiger zu finden sind als in der Verwandtschaft geistig Normaler. Allein viel darüber hinweg sind wir in der Psychiatrie nicht gekommen. Vor allem hat es bisher fast völlig an dem Bestreben gefehlt, Regelmäßigkeiten oder gar Gesetzmäßigkeiten der Vererbung geistiger Abnormität festzustellen. Allein Kant schrieb schon 1786 die beherzigenswerten Worte: „Ich behaupte aber, da B in jeder besonderen Naturlehre nur so viele eigentliche Wissenschaft angetroffen werden kann, als darin Mathematik anzutreffen ist."

In diesem Geiste des Philosophen arbeiten nun seit etwas über ein Jahrzehnt die vererbungsexperimentelle Zoologie und Botanik.

Sie haben die vor geraumer Zeit (1866) von Gregor Mendel, dem Augustinerpater am Königinnenkloster zu Brünn, veröffentlichten Vererbungsregeln gewissermaßen wiederentdeckt und arbeiten in emsiger Weise an ibrer Nachprüfung und Verbesserung und an ihrem Ausbau.

An dieser experimentellen Bastardforschung ist die Psychiatrie bisher leider im großen und ganzen achtlos vorübergegangen. Allerdings hat bei Tieren und Pflanzen das Experiment, im Verein mit scharfsinniger Beobachtung und genialer Begabung auf dem Gebiete schöpferischer Hypothese, den Fortschritt gebracht. Der Mensch bietet sich 
in dieser Weise dem Versuch nicht dar. Allein er untersteht doch auch einem Experimentator. Ihn lenkt die unsichtbare Hand des Schicksals, welche den Menschen zum Menschen verbindet und welche diesem Geschlechtsbande, nicht anders als der Tierexperimentator es auch tut, Generation auf Generation entspringen und zu großen Familien auswachsen läßt, deren Glieder in festen, wenn' auch vielseitig determinierten Blutsverwandtschaftsbeziehungen zueinander stehen und aus diesen Fesseln zeitlebens nicht loskommen, so sehr sie selbst sich das

\section{Mendelsche Prävalenz und Spaltungsregel}

bei Pisum sativum. (Vererbung der Erbsenblütenfarbe.)

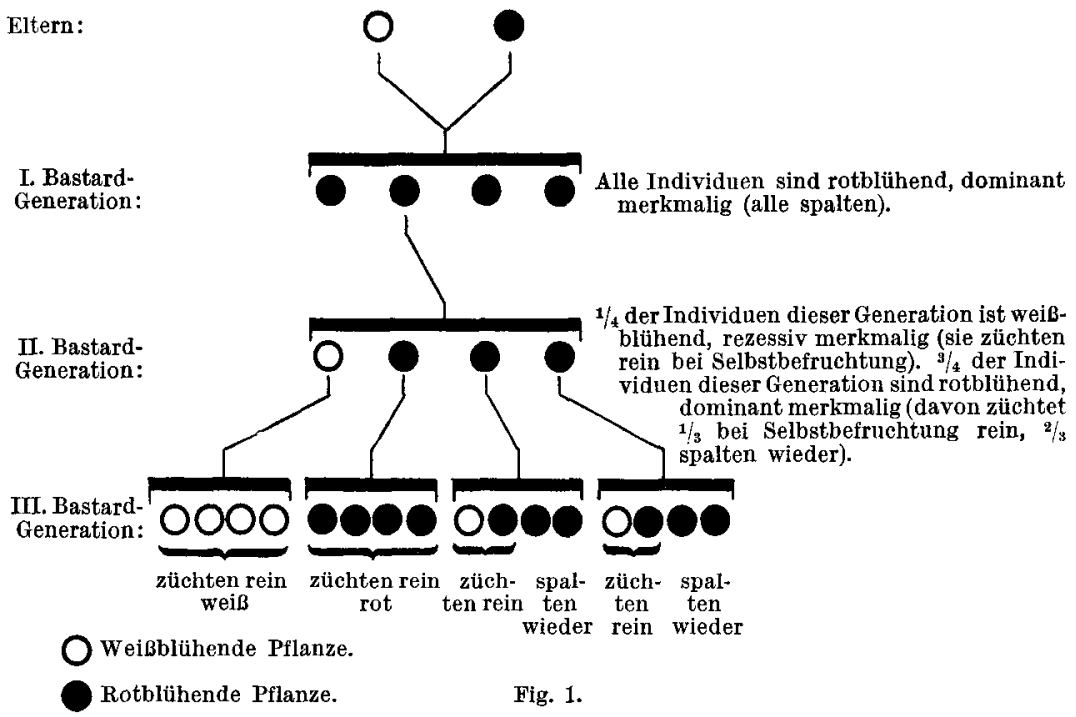

auch oft einbilden. Ihnen ist ihr Platz innerhalb der durch die Abstammung gegebenen Familienstruktur für immer angewiesen.

Im Studium dieser Familienstruktur, in der durch viele Generationen hindurch systematisch verfolgten Familienforschung ist beim Menschen ein fast vollgültiger, wenn auch weit langsamer zum Ziele führender Ersatz für das Vererbungsexperiment bei Tier und Pflanze gegeben.

Aus diesem ersten Grunde - wissenschaftliche Erforschung der Vererbung der Geistesbeschaffenheit beim Menschen - soll der Psychiater methodische Familienforschung treiben.

Bevor ich die Grundzüge dieser erörtere, sei das Prinzip der Mendelschen Vererbung dargelegt.

Die Lehre Mendels geht aus von den Ergebnissen der Kreuzung der weißblühenden mit der rotblühenden Erbse (Fig. 1) und 
hat zur Aufstellung von drei bei der Rassenkreuzung in weitem Umfang gültigen Vererbungsregeln und zu einer Erklärungshypothese geführt.

Benützt man zur Bastardierung zwei reingezüchtete Erbsenrassen der genannten Art, die sich also hinsichtlich eines bestimmten Merkmals, der Blütenfarbe, voneinander unterscheiden, so kommt im reinen Mendelschen Falle bei den Nachkommen, d. h. den Gliedern der ersten Bastardgeneration, nur der eine der beiden korres pond ierenden oder antagonistischen oder allelomorphen Charaktere (die zusammen auch mendelndes Merkmalspaar genannt werden) zum Vorschein, im Falle der Erbse: die rote Blütenfarbe. Alle Bastarde erster Generation sind also rotblühend. Dieses eine der beiden k on k u r rierenden elterlichen Merkmalspaare (hier rot) schlägt also in den nächsten Nachkommen ganz durch, es siegt ob, es dominiert, es herrscht vor, ja es herrscht allein, es prävaliert über das Merkmal des andern Elters (weiß), welches umgekehrt unterliegt, zurückweicht, sich rezessiv verhält. Das ist das Phänomen der Mendelschen Dominanz und Rezessivität oder die Prävalenzregel.

Man bezeichnet sie auch als Unifor mitätsregel nach dem gleichartigen, uniformen Aussehen der ersten Bastard- oder ersten filialen Generation (F. 1), da nur die Uniformität dieser Generation als Regel gelten kann, währenddem die ausschließliche Dominanz oder Prävalenz des einzelnen Merkmals in der ersten Generation, wie sich allmählich herausgestellt hat, doch nur einen Spezialfall darstellt.

Warum das eine Merkmal über das andere siegt, ist noch unbekannt. Es handelt sich um eine Tatsache, mit der die Mendelsche Regel als etwas Gegebenem rechnet und die sie nicht erklären will. Eine Gesetzmäßigkeit etwa in der Richtung, daß die phylogenetisch älteren die jüngeren unterdrücken würden, hat sich noch nicht herausgestellt, wenn auch im allgemeinen die komplizierten Eigenschaften die einfacheren in der ersten Bastardgeneration zu unterdrücken vermögen.

Paart man nun die einheitlich roten Bastarde der ersten Generation unter sich, so entstehen als Nachkommen eines solchen Bastards weiße (R-Formen $=$ Rezessivformen) und rote $($ D-Formen $=$ Dominanzformen) Bastarde zweiter Generation in dem gan z bestimmten Zahlenverhältnisse von 1:3. Das Rot der ersten Bastardgeneration hat sich also in der zweiten Bastardgeneration wieder in das elterliche Weiß und Rot zerspalten. Die Spaltungsgeneration ist gebildet.

Das ist das Phänomen der Mendelschen Spaltungsregel (Fig. 1. Die zweite Bastardgeneration entsteht aus der ersten Bastardgeneration durch Spaltung), weil eine Spaltung der in den Bastarden erster Generation verbundenen Anlagen eingetreten ist.

Die weißblühenden Individuen, $d . h$. der vierte Teil aller Glieder 
der zweiten Bastardgeneration, züchten bei Selbstbefruchtung rein weiß.

Von den rotblühenden Erbsen der zweiten Bastardgeneration, welche zusammen $3 / 4$ aller Bastarde zweiter Generation ausmachen,

Kreuzungsversuch der rot und weibblühenden Erbse.

Anlagen-Kombinationen bei der Gameten- und Zygotenbildung.

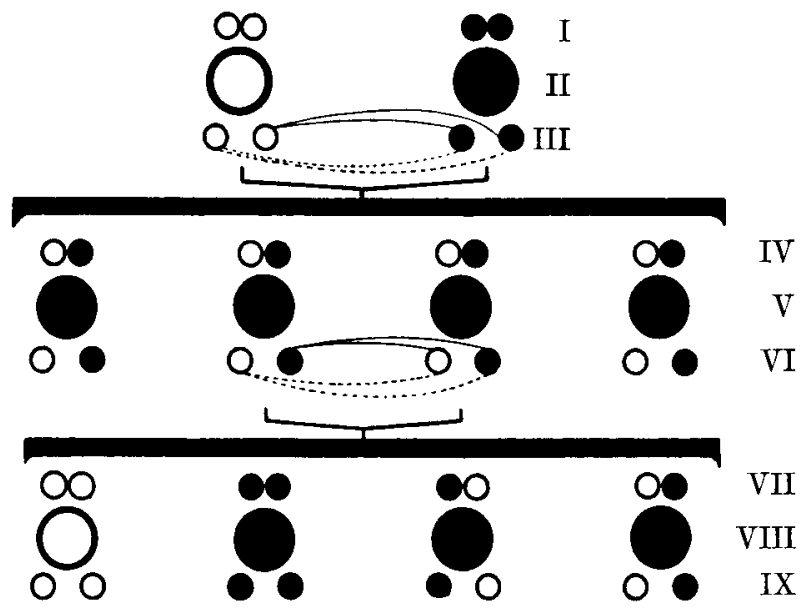

QQ Keime, die ausschlieBlich die Anlage zur weißen Blütenfarbe enthalten.

Keime, die sowohl̈ die Anläge zü weiß̈en, als auch zür roten Blütenfarbe enthalten.

Fig. 2.

Zu Reihe I: Keime, aus denen die Eltern hervorgehen, sie enthalten die eine Anlage, weiB oder rot, sie sind "keimrein", ho mozyg ot.

II: Die zwei Eltern, die aus den obigen Keimen hervorgehen.

III: Anlagereine, homozygote Keime, welche von den Eltern produziert werden.

IV: Resultat der Kombination der in den elterlichen Keimen enthaltenen Anlagen: Heterozygote oder anlageverschiedene Keime.

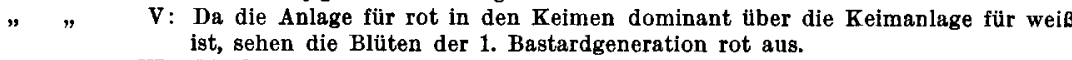

" V: Da die Anlage für rot in den Keimen dominant über die Keimanlage für wei

" " VI: Die 2 Anlagen, welche in den heterozygoten Keimen sind und von den Eltern herstammen, trennen sich wieder, so daß eine Hälfte der Geschlechtszellen nur noch die Anlage zu weib, die andere Hälfte nur noch die Anlage zu rot enthält.

VII: Die 4 Kombinationen von Anlagen, welche durch die Kreuzung zweier Bastarde

" VII: Die 4 Kombinationen von Anlagen, welche durch die Kreuzung zweier Bastarde
der 1. Bastardgeneration entstehen (2 heterozygote und 2 homozygote Keime). Keimen weißblïhende und rotblühende Individuen im Verhältnis von $1: 3$.

" IX: Das von der 1. Bastardgeneration übernommene Anlagematerial wird wieder zerlegt; 1 weißes Individuum erzeugt, weil es selbst aus Keimen mit ausschlie Blicher Anlage zu wei $\beta$ hervorgegangen ist, wieder nur Keime mit Anlage zu weib, 1 zweites rotes Individuum nur Keime mit Anlage zu rot und 2 rote Individuen Keime mit beiden Anlagen.

züchtet $1 / 3$ ebenfalls rein, aber rotblühend, die übrigen $2 / 3$ der rotblühenden Bastarde zweiter Generation spalten bei Selbstbefruchtung oder Kreuzung untereinander wiederum in weißblühende und rotblühende Bastarde dritter Generation, und zwar wiederum im Verhältnis von $1: 3$, und so fort. 
Es entstehen also bei der reinen Mendelschen Vererbung keine Mischprodukte aus den ererbten Eigenschaften, sondern diese tauchen in allen Generationen unverändert immer wieder auf, und zwar in gesetzmäßigen Zahlen-Proportionen. Die Mendelsche Vererbung wird daher auch die spaltende oder alternative genannt, weil eben die elterlichen Eigenschaften nicht verschmelzen (wie bei der intermediären, s. hinten), sondern unter den Nachkommen alternierend auftreten oder von einer Generation zur andern sich immer wieder in die elterlichen Ausgangscharaktere, in die D.- und R.-Individuen, aufspalten.

Zur Erklärung dieser regelmäßigen Zahlenproportionen im Dominieren, Verschwinden und Wiederauftauchen der Erbqualitäten hat Mendel eine ingeniöse und einleuchtende $H y$ pothese aufgestellt. Er nahm an (Fig. 2), daß die (reingezüchteten) Eltern (Reihe II) aus Keimen oder Geschlechtszellen oder Gameten mit nur einer Anlage (I), entweder zu weiß oder zu rot, entstehen und auch wieder einanlagige Geschlechtszellen oder Gameten bilden (III). Verbinden sich diese zweierlei Keime des väterlichen und mütterlichen Elters miteinander, so entsteht ein befruchteter Keim, eine Zygote, in welcher nun also 2 verschiedene Anlagen, nämlich zu weiß und rot (IV), eingeschlossen sind und aus denen dann der Bastard erster Generation entsteht (V). Allein, wenn dieser Bastard erster Generation seine Geschlechtszellen oder Gameten (VI) bildet, tritt eine Zerspaltung der bei seiner eigenen Entstehung miteinander vereinigten verschiedenen, d. h. antagonistischen (weiß und rot) Anlagen des Merkmalpaares ein.

Es wandert gewissermaßen je nur eine Farbenanlage in einen Keim, so daß dann die eine Hälfte aller Geschlechtskeime (Gameten) des Bastards erster Generation nur noch die Anlage für das dominante (rote), die andere Hälfte nur noch die Anlagen für das recessive (weiße) Merkmal in sich schließt (Mendels Hy pothese von der Reinheit der Gameten). Paaren sich dann die Geschlechtszellen zweier solcher Bastarde erster Generation, so müssen Zygoten in den Kombinationen und Proportionen wie in Reihe VII entstehen und so fort.

Diese ,Spaltung“ oder Verteilung der antagonistischen Merkmale auf verschiedene Keimzellen findet vielleicht während der Reduktionsteilung der reifenden Eier und Samenfäden statt, vielleicht aber auch während der Synapsis.

Aus Zygoten, welche nur die Anlage zu weiß enthalten, können nun natürlich nur weiß aussehende Blüten entstehen, nur rote Blüten aus Zygoten mit ausschließlicher Anlage zu rot. Rein- oder gleichanlagige befruchtete Keime heißt man homozygote. Aus befruchteten Keimen (Zygoten), welche die Anlage sowohl zu weiß als 
zu rot, also verschiedene Anlagen, enthalten und welche deshalb heterozygote genannt werden, entstehen rotblühende Erbsen, weil Rot in diesem Spezialfalle dominant ist, nach der Prävalenzregel also maßgebend für das Aussehen des aus einem solchen Heterozygotenkeime hervorgehenden Individuums ist.

Die Mendelsche Regel in dieser einfachen Form und die einfache Erklärung der bei ihr zu findenden Zahlenproportionen hält nun auch Stich bei einzelnen Anomalien des Menschen. Der 5 Generationen umfassende Stammbaum der Träger einer solchen mendelnden Eigenschaft, der Brachydactylie, ist hier abgebildet (Fig. 3).

Auch hier finden sich 2 konkurrierende Merkmalspaare, nämlich abnorm (brachydactyl) und normal (d. h. nicht brachydactyl); allein

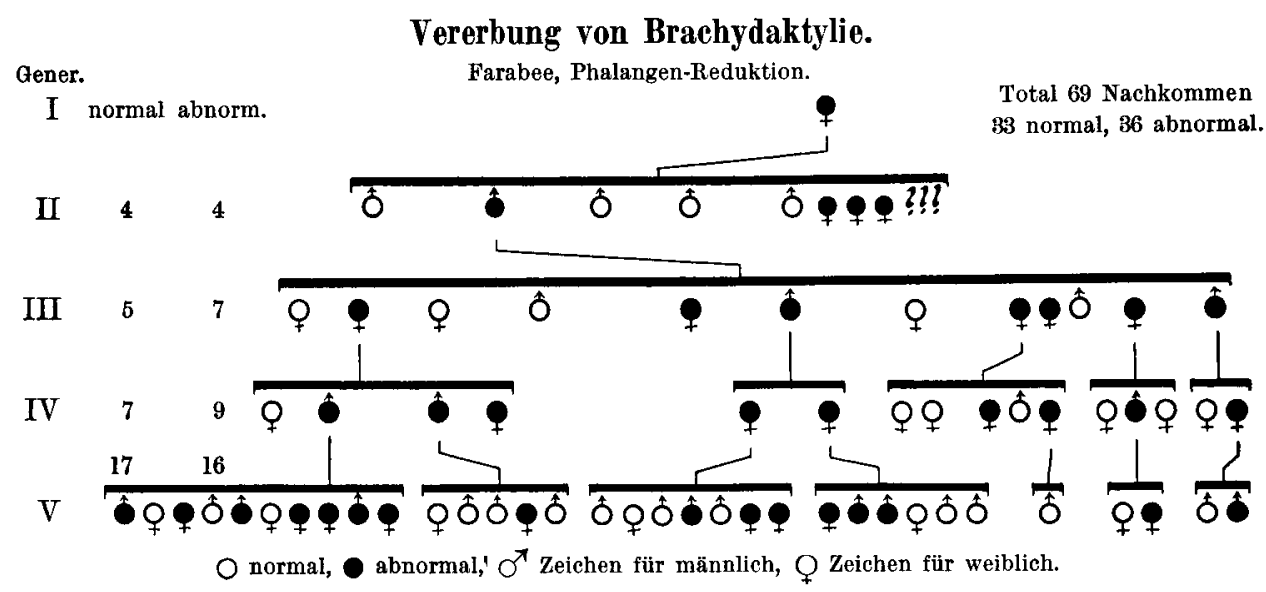

Fig. 3.

vieles ist doch anders. Zwar züchten die Nichtbrachydactylen - vorweggenommen sei, daß sie stets nur ebenfalls Normale heirateten auch rein, denn alle ihre Nachkommen sind normal mit Bezug auf dieses Merkmal, verhalten sich also ganz wie die weißblühenden Erbsen. Auch treten die Träger des anderen Merkmals, die Brachydactylen, stets in jeder Generation auf, ebenso wie die rotblühenden Erbsen. Allein sie schlagen in keiner Generation, ja sogar (es sei denn, daß es sich, wie in einem Falle, um eine zu geringe Zahl [2] von Nachkommen handelt) in keiner einzelnen Familie mit ihrer Eigenschaft völlig durch, und in allen Generationen ist die Proportion von Normal zu Abnorm nicht wie 3:1 oder 1: 3, wie im Erbsenfalle, sondern ganz nahe an 1: 1. (In der zweiten Generation wie 4:4, in der dritten 5:7, vierten $7: 9$, fünften 17: 16.) Auch die Gesamtsummen der Normalen und Abnormen verhalten sich wie 33:36, also, wie wir annehmen dürfen, wie $1: 1$.

Die Erklärung dieser Abweichungen gebe ich in Fig. 4. 
Nimmt man an, daß die Brachydactylie in der betreffenden Familie ein dominierendes Merkmal ist, setzt man als Stammeltern, wie im

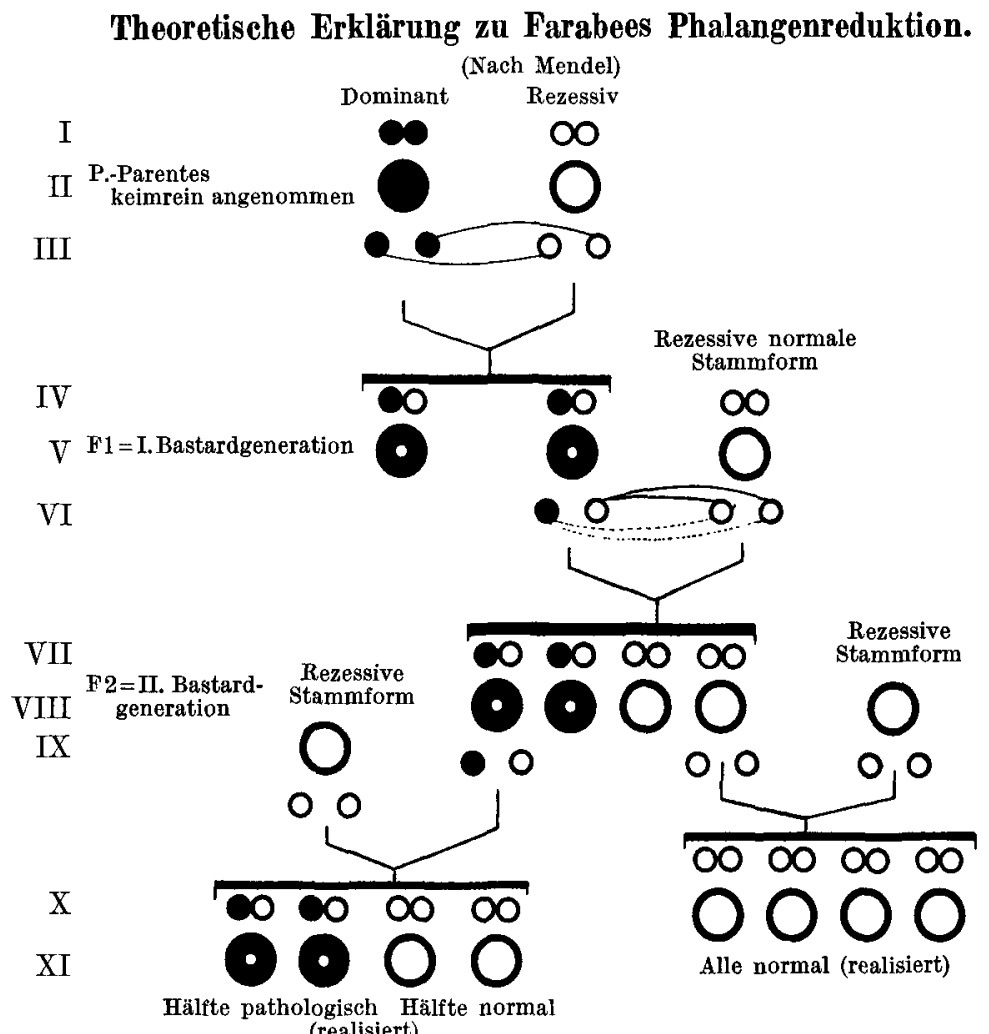

Nicht realisiert in der Wirklichkeit sind folgende Kombinationen, weil ihre Voraussetzungen (Keimreinheit der Abnormen und Abnormenheirat unter sich) in Wirklichkeit ebenfalls fehlen. abnorm, rezess. abnorm, abnorm, abnorm, abnorm, abnorm, abnorm, keimrein Stammform keimrein keimrein heterozygot keimrein heterozygot heterozygot

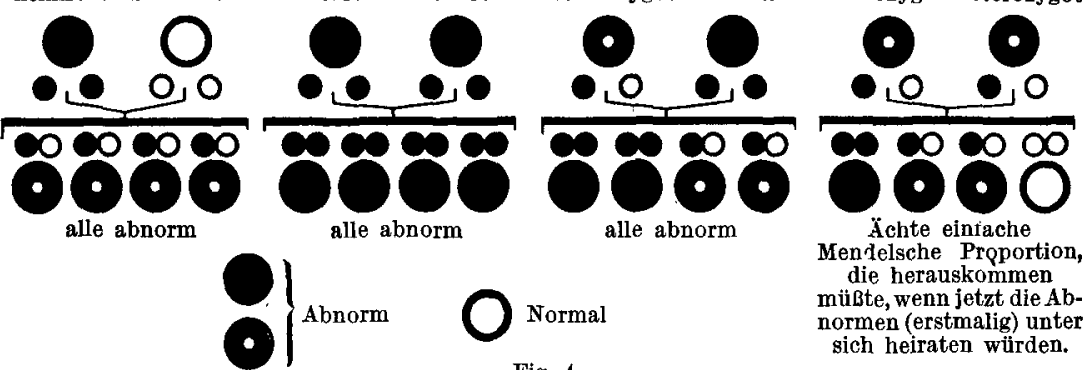

Fig. 4.

Falle der Erbsen, 2 reingezüchtete Individuen (welche in Wirklichkeit allerdings nicht zu finden sind, weil alle Abnormen einen normalen Vater oder eine normale Mutter hatten) und verfolgt man dann hier, nach 
Analogie der figürlichen Darstellung der Erbsenkreuzung, die Anlagenkombinationen, so werden auch die Zahlenproportionen ohne weiteres verständlich.

In der Familie dieser Brachydactylen sind alle bekannten Abnormen aus je einem Gesunden und kranken Elter hervorgegangen, sie können also nicht anders als heterozygot sein (Reihe $V$, dunkle Kreise mit einem weißen Punkt in der Mitte, welcher die verborgene Anlage zu weiß markieren soll). Die von ihnen gebildeten Anlagen (Reihe VI) spalten nun aber wieder und bilden bei ihrer Verbindung mit den rein normalen Anlagen oder gesunden Homozygoten der gesunden Partner (recessive Stammform) die Anlagenkombinationen der Reihe VII, d. h., wie aus der 4 fachen Kombination aus Reihe VI leicht verfolgt werden kann, zur Hälfte heterozygote Keime (die 2 verschiedenen Anlagen im Keim) und zur Hälfte homozygote Keime (die 2 normalen Anlagen im Keime).

Die aus verschiedenanlagigen Keimen (Heterozygoten) hervorgegangenen Individuen der Reihe VIII können dann nach der Prävalenzregel auch wieder nur abnorm (schwarzer Kreis) aussehen, weil ja abnorm hier dominant ist.

Die aus Keimen mit gleichen Anlagen, und zwar zu normal (Homozygot), hervorgegangenen Individuen der Reihe VIII (weiße Kreise) können, da die andere (dominante, abnorme) Anlage, die in der Anlagenkombination, aus der sie entstanden sind, völlig fehlte, die recessive normale also allein zurückgelassen hat, nur das recessiv-merkmalige, d. h. das normale Aussehen annehmen.

Hieraus ergeben sich Konsequenzen, die für Vererbung und Belastung von der allergrößten theoretischen und praktischen Wichtigkeit sind.

Es gibt Charaktere, z. B. Anomalien, auch beim Menschen, welche sich ganz kontinuierlich, ohne Unterbrechung, von einer Generation zur anderen direkt hindurch vererben, und zwar so, daß die eine Hälfte der Nachkommen wieder abnorm, die andere Hälfte normal ist. Keine Verbindung mit normalem, keine Rückkreuzung mit ,frischem“" Blut fremder Familien (recessive Stammform) ist imstande, für die Reihe absehbarer Generationen die Anomalie zum Verschwinden zu bringen. Aus dem Studium anderer Anomalien, die sich ebenso vererben, geht aber andererseits auch hervor, daß mit Bezug auf das Auftreten der Anomalie in der Nachkommenschaft nicht etwa eine Verstärkung oder Häufung eintritt, wenn Ehen zwischen Abnormen und Normalen derselben Familie geschlossen werden. Auch hier finden sich dieselben Proportionen, was ja auch bei einer Betrachtung der Fig. 4, ohne weiteres verständlich ist; denn die Normalen auch der eigenen Familien sind mit Bezug auf das genannte Merkmal recessive Homozygote urd müssen dieselben Anlagekombinationen ergeben wie die recessiven Homo- 
zygoten der fremden Familien. Blutsverwandte Ehen zwischen einem dominant-abnormen Heterozygoten und einem recessiv-normalen Homozygoten haben also mit Bezug auf das eine mendelnde Merkmal dieselben, keine schlimmeren Wirkungen als die Verbindungen des Heterozygoten mit einem Homozygoten fremden Stammes.

$\mathrm{Ja}$, wir wissen aus anderen Stammbäumen, daß homozygote Eltern aus derselben Familie mit Bezug auf ähnlich sich vererbende Merkmale ebenfalls lauter normale Nachkommen haben, genau wie die Verbindungen zwischen nicht blutsverwandten Homozygoten. Es kommt also hier praktisch nur darauf an, wie die blutsverwandten Partner selbst aussehen, nicht darauf, ob der eine oder beide Partner aus schwer belasteten oder unbelasteten Familien stammen. Mit anderen Worten, die enorme „Belastung“ besagt hier, wenn die Anomalie dominant geht, für den Erbwert, der in dem normal aussehenden Glied der kranken Familie schlummert, gar nichts. Der Gesunde einer solch schwer kranken Familie wird stets nur (mit Bezug auf diese Eigenschaft!) normale Nachkommen haben.

Der enorme praktische Wert dieser Erkenntnis, die man in die Worte zusammenfassen kann „einmal frei, immer frei“ leuchtet ein. Der bisherige vage Begriff der erblichen Belastung und des Verhängnisses blutsverwandter Ehen gewinnt hier bestimmte Gestalt.

Er verliert seine Schrecken völlig für die normalen Varianten der Familie, um sie allerdings um so deutlicher, unentrinnbarer und mathemathisch unmißverständlicher für die Nachkommenschaft der kranken Varianten der Familie aufzuzeigen.

Das äußere Aussehen der Varianten bietet hier einen sicher führenden Anhalt für den inneren, den Erbwert der Varianten und somit für das Aussehen der Nachkommenschaft.

Wie man sieht, in diesem Spezialfalle, eine außerordentlich einfache, exakte, zuverlässige Antwort auf die uns so lebhaft interessierende Frage: „Wie regeneriert sich sicher eine kranke, schwer belastete Familie?"

Die praktischen Schlußfolgerungen gehen aber viel weiter, nur eine wichtige soll hier noch genannt werden.

Es kann in demselben Stamme mit dominant gehender Anomalie eine höherwertige Eigenschaft, ein Talent recessiv gehen (s. weiter unten).

In ähnlichen Fällen kann also eine gewisse Inzucht unter den anomaliefreien Individuen geradezu erwünscht sein, da sonst die betrübende Sicherheit besteht, daß der für den Stamm typische Anlagevorzug bei definitiver Fremdkreuzung als Erbmerkmal definitiv verloren geht.

Eine interessante und praktisch hoch bedeutsame Modifikation der Mendelschen Regel ist die unvollkommene Dominanz (die man

z. f. d. g. Neur. u. Psych. O. viI. 
im Einklang mit Mendelschen Vorstellungen auf ein Zusammenwirken einer größeren Anzahl von Erbfaktoren zurückführen kann, siehe weiter hinten, Grundfaktor-Supplement-Theorie), weil sie ebenfalls an Gesetzmäßigkeiten gebundene Verknüpfungen darstellt mit dem, was äußerlich als intermediäre Vererbung zutage tritt (wo also im Bastard die Eigenschaften der Eltern in Mittelstellung, als Mischresultat gewissermaßen, ausgeprägt sind), somit auch diese scheinbar ganz außerhalb des Geltungsbereichs Mendelscher Vererbung stehende Art der Vererbung, für viele Fälle wenigstens, in jene mit einbezieht und weil hier der für den Menschen praktisch so wichtige Fall gegeben

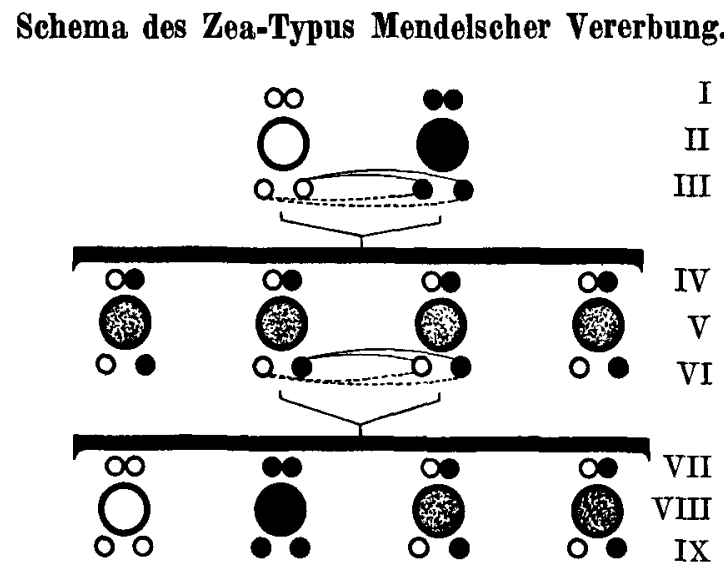

Q Keime, die ausschließlich die Anlage zur weißen Blittenfarbe enthalten.

O. Keime, die sowohl̈ die Anlage zur weiß̈en als auch zur roten Blitenfarbe enthalten.

Weiß blihendes Individuum.
Rot $"$
Rose $"$

ist, daß man dem dominanten Heterozygoten hier auch äußerlich, ohne Vornahme einer Probezucht, deutlich den Unterschied vom dominanten Homozygoten ansieht, was im Erbsenfalle nicht zutrifft.

Ein schönes Beispiel hiervon ist die Kreuzung einer roten mit einer weißen Mirabilis Jalappa (Wunderblume), in Fig. 5. Sie gibt einen Bastard (unvollkommener, dominanter Heterozygoter) mit intermediärer Rosafärbung, und dieser liefert bei Selbstbefruchtung dreierlei Nachkommen, nämlich eine Pflanze (vollkommen dominanter Homozygoter) mit Blüten, die genau so intensiv rot sind wie das Rot des einen Großelters, 2 Pflanzen (unvollkommen dominante Heterozygote) mit Rosablüten, wie die des Elters, und eine weiße Pflanze 
mit Rücksicht auf die Psychiatrie.

Anlagen-Kombinationen bei Rezessivität der Anomalie.

I
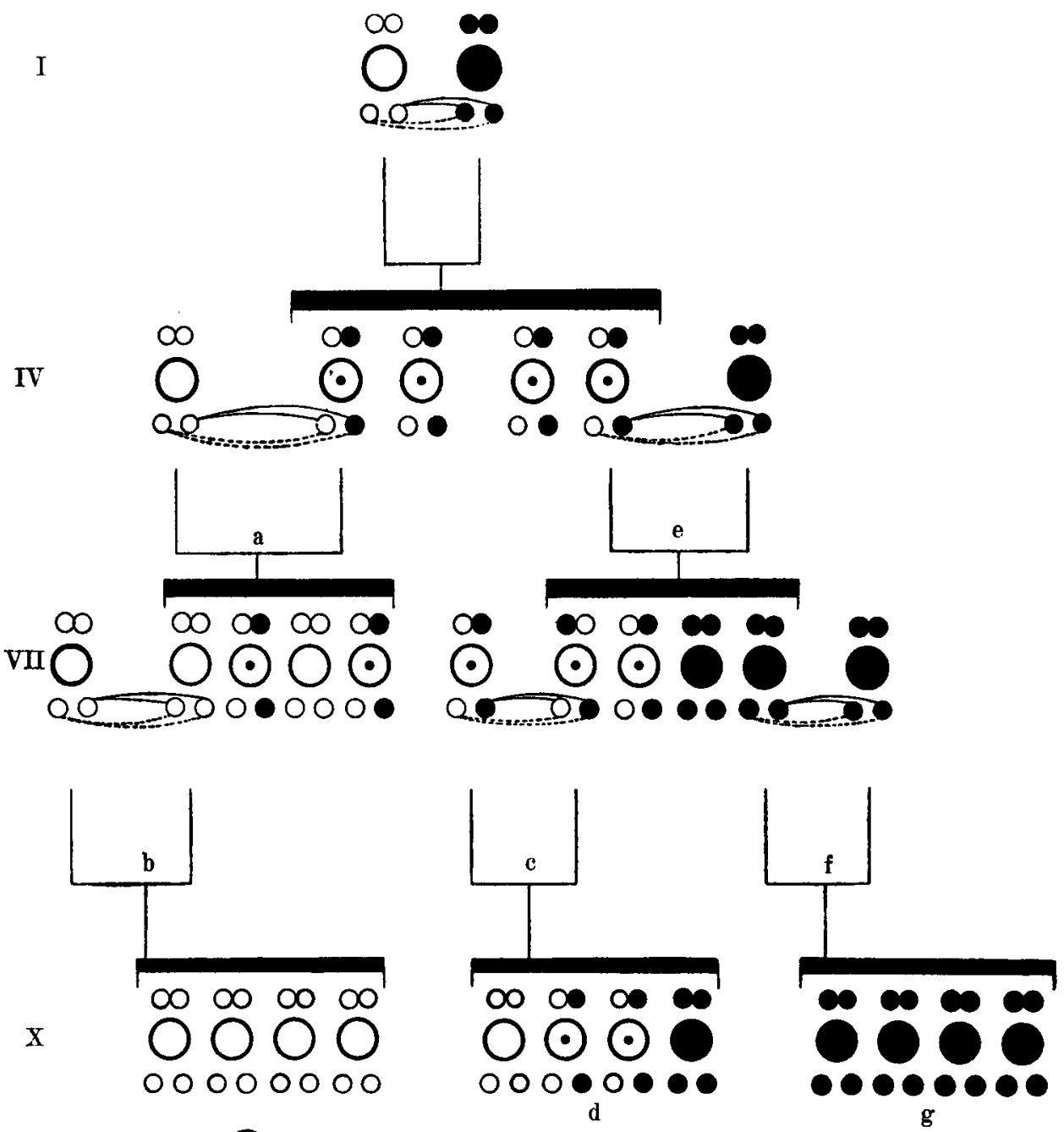

Dominant merkmalig, homozygot. Normal

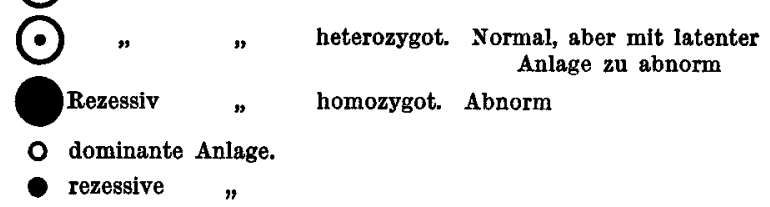

Fig. 6.

(recessiver Homozygoter), so weiß wie der andere Großelter. Die rosafarbenen spalten wieder, die rote und weiße Blüte züchten rein.

Diese interessante Modifikation ist der besonders bei Pflanzen sehr 
weit verbreitete Zeatypus der Mendelschen Vererbung, so benannt, weil er zuerst von Correns beim Mais beobachtet wurde.

Aus seinem Vorkommen geht wohl hervor, daß die Prävalenz an der Mendelschen Regel nicht die Hauptsache ist. Das Wesentliche am Mendelschen Vererbungsmodus ist, daß die mendelnden, antagonistischen Merkmals paarekeinedauernden Verbindungen eingehen, sondern immer wieder unverändert zum Vorschein kommen.

Ganz anders stehen die Dinge, wenn eine Abnormität nicht dominant, sondern recessiv sich vererbt, also gegenüber dem mit ihm kon-

\section{Schema rezessiver mendelnder Vererbung.}

Belastung (collaterale) der Probandinnen durch eine Urgroßtante bei a und einen Urgroßonkel bei $b$.

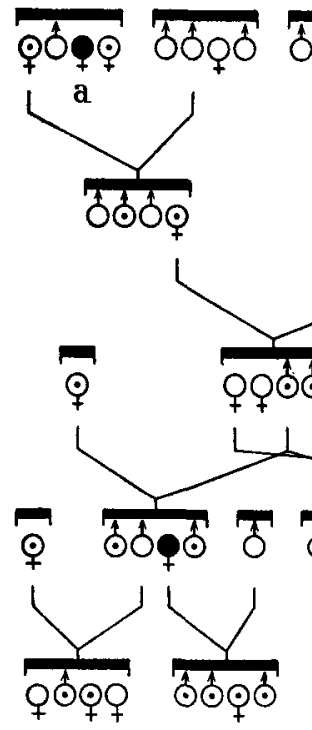

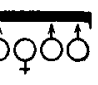

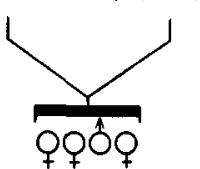

૧ฺớ
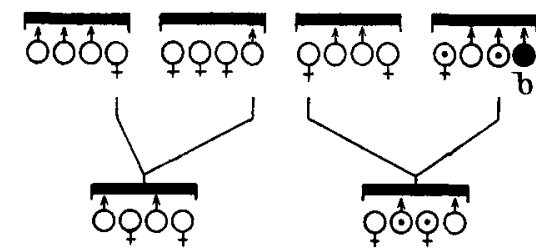

IV
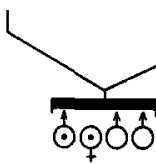

Fig. 7.

kurrierenden Merkmal: normal, d. h. von der betreffenden Anomalie frei, im Vererbungsgange unterliegt.

Ein Schema der 6 Möglichkeiten bei einfacher, recessiv mendelnder Vererbung gebe ich in Fig. 6. Hier ist, umgekehrt wie in Fig. 4, die recessive, abnorme Form schwarz gezeichnet (Fig. 6).

Wie man sieht, unterliegt sie völlig in der Generation (Reihe IV), die aus der Paarung eines dominanten Homozygoten mit einem recessiven Homozygoten (Reihe I) hervorgeht. Alle Individuen (Reihe IV) sind gesund, trotzdem sie durch den einen Elter belastet sind. Die Abnormität unterliegt auch zumeist, besser, kommt nicht zum Vorschein, wenn dominant (normal) mit dominant sich paart 
(Reihe IV, a, dominant homozygot mit dominant heterozygot, Reihe VII, b, dominant homozygot mit dominant homozygot).

Mit einer wichtigen Ausnahme, denn sie kommt wieder zum Vorschein, wenn, wie in Reihe VIIc, zwei dominante Heterozygote sich paaren, also 2 gesunde Individuen mit zwiefacher, nämlich normaler und abnormer, Anlage. Dann ist $1 / 4$ der Nachkommenschaft recessiv homozygot, also abnorm (Reihe $\mathrm{X}$, bei d).

Paart sich aber ein recessiv Homozygoter (Abnormer) mit einem dominanten Heterozygoten (Reihe IV, bei e), so ist die Hälfte der Nach-

\section{Schema rezessiver mendelnder Vererbung.}

Direkte diskontinuierliche Belastung: 1. durch einen Urgrobvater (für Probandin $\mathrm{I}$ ), 2. durch einen Urgroßvater und den Vater (fürr Proband II).

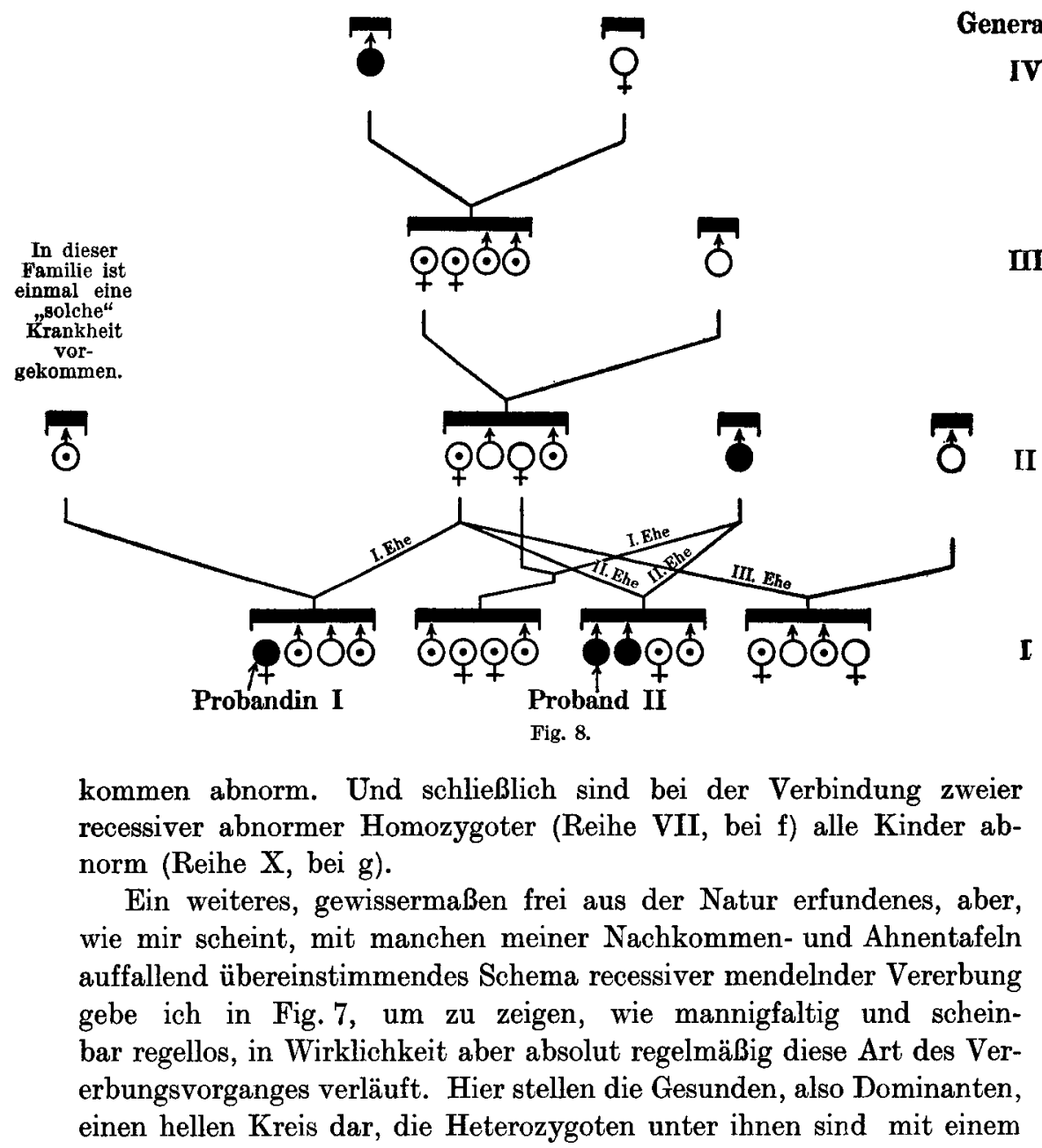


zentralen Punkt versehen, um anzudeuten, $d a B$ in ihnen die recessive, pathologische Anlage schlummert, wenn sie selbst auch ganz gesund aussehen.

Die recessiven Homozygoten, also Abnormen, sind hier voll schwarz gezeichnet, da sie ja die Anlage zu gesund überhaupt nicht enthalten, also nur recessives Äußeres haben können.

Bei der Betrachtung dieses Schemas, dem sich ein ähnliches (Fig. 8) anschließt, und beim Vergleich mit Fig. 3 ergeben sich die größten Verschiedenheiten.

Vor allem ist, im Gegensatz zum Falle der Fig. 3 zu konstatieren, daß die Kontinuität der Vererbung durchaus nicht die Regel ist und daß hier auch keineswegs die Regel gilt: „Einmal frei, immer frei.“ Hier herrscht, scheinbar ohne Gesetzmäßigkeit, bald die „direkte", bald die ,indirekte“, bald die „kollaterale", bald die "kontinuierliche", bald die ,dis kontinuierliche" Vererbung vor.

Dieselben Gesunden haben mit Gesunden bald nur Gesunde, bald aber auch kranke Nachkommen, und ebenso ergeht es da, wo eines der Eltern krank ist. Gesunde aus belasteten Familien können ebenso gesunde Nachkommen haben, als Gesunde aus unbelasteten Familien. Nur 2 Phänomene bestehen beiderseits zu Recht: $\mathrm{Paar}$ ungen zw ischen Gesunden aus unbelasteten Familien zeigen keine kranken Nachkommen, und wo beide Eltern krank sind, sind auch alle Nachkommen krank.

Allein ein Blick auf die Figuren lehrt, daß auch die scheinbaren Unregelmäßigkeiten, die ,A usnahmen“, wie man sich oft ausdrückt, ihren ganz bestimmten Grund haben und ebenfalls Regeln folgen. Wenn zwei Gesunde kranke Kinder haben, so haben sie stets eines unter 4, 2 unter 8 und sind stets keine keimreinen Gesunden, sondern Heterozygoten, stammen also selbst von Ahnen, von denen einmal einer krank war.

Haben Gesunde aber gesunde Kinder, so ist mindestens 1 Elter keimrein, d. h. ein Homozygoter, unter dessen Vorfahren überhaupt nie eine ähnliche Krankheit vorkam, oder doch nur bei den indirekten Vorfahren (Großeltern, Urgroßeltern) oder bei Kollateralen, nie aber bei den Eltern.

Auch in den Fällen, wo ein kranker Elter gesunde Kinder hat, muß der andere Elter ein homozygoter Gesunder sein, darf also keine Anlage zu krank enthalten.

Wo ein Kranker mit einem Gesunden kranke Kinder hat, da finden sich diese an Zahl im Verhältnis zu den Gesunden vertreten wie 1: 1. Die Hälfte der Kinder ist krank. In diesem Falle ist aber der gesunde Elter ein heterozygoter Gesunder.

Zur Übersicht über das Gesagte und besonders über die verschie- 
denen Erkrankungsproportionen, die zu erwarten sind, je nachdem sich eine Abnormität dominant oder recessiv nach Mendel vererbt, stelle ich die 6 Modalitäten bei jeder Vererbungsart in Fig. 9 und 10 nebeneinander.

Aus dieser Zusammenstellung kann man sich auch ein gutes Bild machen, wie klar und gesetzmäßig und doch wie verborgen und früher so unregelmäßig erscheinend die Beziehungen zwischen Belastung, äußerem Aussehen der Varianten und Erbwertigkeit sind.

Vererbungsproportionen bei Dominanz der Anomalie.

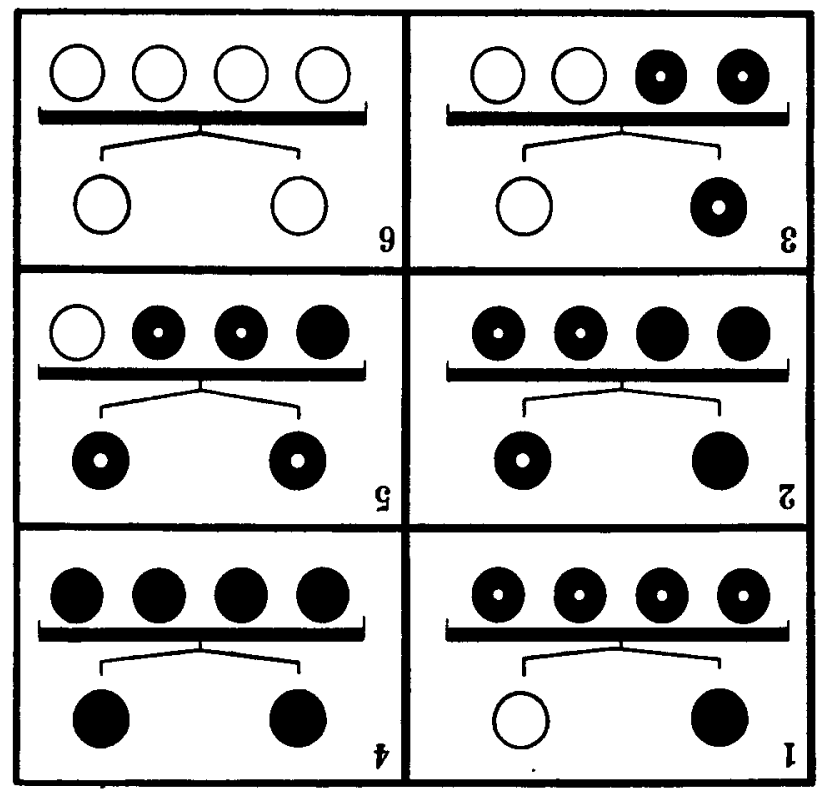

Krank, dominant homozygot

- Krank, dominant, heterozygot

gesund, rezessiv, homozygot

Fig. 9.

Denn Individuen können identische Vorfahren, zuweilen allem Anschein nach identische Charaktere haben und dennoch in gametischer Zusammensetzung ganz verschieden sein (in Fig. 10 haben in Rubrik 5 und 6 die Kinder identische Vorfahren, und 7 von 8 Nachkommen sehen sich äußerlich gleich, haben aber verschiedene Keimzusammensetzung).

Umgekehrt können Individuen identisch sein in ga metischer Beziehung und dennoch verschiedene Vorfahren haben (Fig. 10, die heterozygoten Kinder der Rubriken 2 und 3). 
Vergegenwärtigen wir uns all das Gesagte, so lassen sich über die einfache Form der recessiven Mendelschen Vererbung folgende charakteristische Hauptpunkte aufstellen.

Im Gegensatz zu dem Falle, wo eine Anomalie dominant geht, unterliegt bei der recessiven Utbertragungsform das Kranke dem Gesunden. Finden wir also bei den Familien, wo eine Anomalie dominant mendelt, sehr viele Abnorme - in den Töchtergenerationen der Fig. 9 17 Abnorme, der Fig. 10 nur 7 Abnorme - und zwar in jeder Generation

Vererbungsproportionen bei Rezessivität der Anomalie.

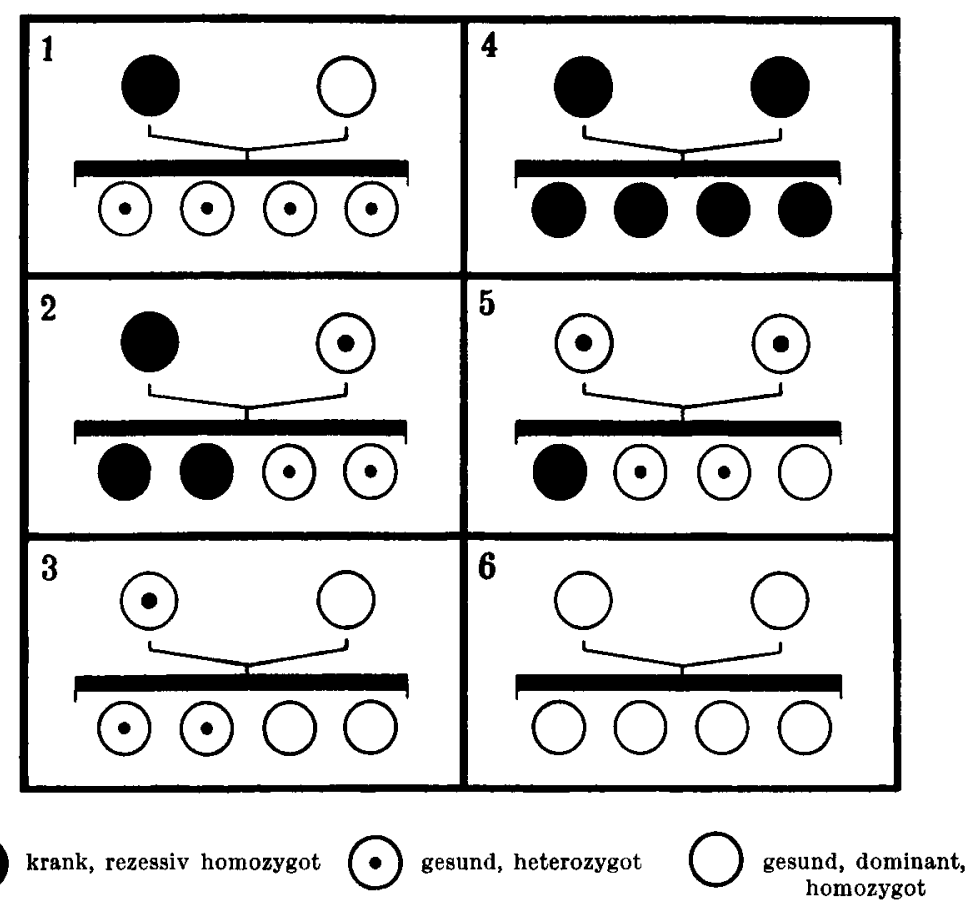

Fig. 10 .

und selbst in jeder Familie, die einen kranken Elter aufweist, so finden wir in den Familien mit recessiv mendelnden Abnormitäten im allgemeinen viel weniger Abnorme, durchaus nicht in jeder Generation und durchaus nicht in jeder Familie.

Ja, und das ist das Hochbedeutsame: Es können 2 (ja 3 und mehr) Generationen übersprungen werden (wie aus Fig. 7 ersichtlich).

Im Gegensatz zum dominanten Modus ist in Familien mit recessiv mendelnden Abnormitäten der äußerlich Gesunde nicht immer auch zugleich keimgesund. Das Äußere führt hier, im Gegensatz zum domi- 
nanten Falle, irre. Bei Verbindungen mit ebenso beschaffenen, im Keime zum Teil krank veranlagten Individuen (recessiven Heterozygoten), seien sie fremden oder eigenen Stammes, tritt daher in der Deszendenz die Anomalie wieder hervor, freilich nur in den Zahlengrenzen, die ihr für diesen Fall durch die Mendelsche Proportionslehre gezogen sind. Es ist nach dem Gesagten auch klar, daß die Chancen für derartige Kreuzungsfolgen ganz besonders schlecht in Ehen zwischen Verwandten sein müssen, die einen und denselben oder mehrere abnorme Vorfahren besitzen.

In Familien, die mit recessiv mendelnden Abnormitäten belastet sind, ist daher die Nachkommenschaft auch von gesunden Verwandtenehen, im Gegensatz zum Falle der dominant gehenden Abnormität, aufs höchste gefährdet, wenn nicht, was natürlich ebenfalls möglich, weil durch die Spaltungsregel gefordert, mindestens der eine Partner des Verwandtenpaares ein gesunder Homozygoter ist.

Auch der kranke Träger einer recessiv gehenden Eigenschaft unterscheidet sich vom kranken Träger einer dominanten Eigenschaft dadurch, daß er in seinen Keimen keine normale Anlage zur Verfügung hat.

Somit sehen wir in bezug auf die Sicherheit, Rückschlüsse von der äußeren Leibesbeschaffenheit auf die Keimanlagenbeschaffenheit zu ziehen, bei dominantem und recessivem Mendeln genau das Gegenteil.

Jeder Gesunde einer dominant vererbenden Familie ist auch keimgesund. Beim Gesunden einer die Anomalie recessiv vererbenden $\mathrm{Fa}$ milie kann das der Fall sein (Fig. 10, Rubr. 3), muß es aber nicht. Durch bestimmte Kreuzungskombinationen und Vergleich der erhaltenen Proportionen stellt der Tier- und Pflanzenzüchter die Homozygie (Keimreinheit) oder Heterozygie fest. Wie wir später sehen, kann das der Mensch durch das Studium lückenloser Nachkommienund Ahnentafeln.

Jeder Kranke einer die Krankheit recessiv vererbenden Familie ist eo ipso auch völlig keimkrank. Dagegen kann der dominant Kranke, nämlich dann wenn der eine Elter normal, auch die Anlage zu Gesund besitzen. Auch das stellt der Tierzüchter durch vergleichende Kreuzungen fest. Beim Menschen ist aber wohl zumeist der eine Elter abnormitätsfrei, womit die Nachkommen als Heterozygote schon bestimmt sind (zur Hälfte Kranke, dominante, zur Hälfte gesunde, recessive Anlagen). Nur wo beide Eltern dominant abnorm sind, treten in der Nachkommenschaft auch Homozygote auf (Fig. 9, Rubr. 2, 4 und 5).

Sahen wir die dominante Anomalie sich unter allen Kreuzungsbedingungen hartnäckig forterhalten, so besteht die Möglichkeit, eine 
recessiv gehende Anomalie durch fortdauernde Rückkreuzung mit einer fremden gesunden Stammform definitiv aus der abnormen Familie zu entfernen. Hier ist eine Auffrischung also bei konsequenter Durchführung sicher, dort unmöglich. Es ist das Gegenstück zum Verhalten der Anomalie bei blutsverwandten Ehen unter Gesunden. In Familien mit dominant gehender Anomalie schadet die Verwandtenehe Gesunder nichts, in solchen mit recessiven Abnormitäten vermehrt sie erheblich die Erkrankungsziffer.

Im Gegensatz zum dominanten Falle besagt also die oft große Belastung in Familien mit recessiver Krankheitsvererbung durch einen kranken Elter (wie z. B. in Fig. 8, durch einen kranken Vater) nichts, wenn der andere Elter auch im Keim gesund ist, also sicher bei Heirat in einen fremden, gesunden Stamm (Fig. 8).

Aber dieselben Vorsichtsmaßregeln müssen sich im Gegensatz zu den Familien mit dominanten Anomalien auch die Gesunden der recessiven Familien auferlegen, selbst wenn die Belastung eine recht entfernte ist (Fig. 7 und Fig. 8).

Die Belastung jeden Grades bedeutet also, wenn nicht Bedacht auf Rückkreuzung mit Gesunden aus gesunden Familien genommen wird, in welchem Falle sie helanglos ist, für weitaus die meisten Glieder aus recessiven Familien (je nach Intensität und Nähe der Belastung für alle, $3 / 4$ oder die Hälfte der Kinder eines Elternpaares) ein großes Verhängnis.

Im dominanten Falle aber hat die Belastung nur für die Nachkommenschaft der Kranken selbst Bedeutung, für diejenige der Gesunden keine.

Es ergeben sich somit prophylaktisch die zwei verschiedenen Hauptgrundsätze:

für die Familien mit dominantgehenden Anomalien:

Kreuzung nur der gesunden Glieder unter sich nach außen oder innerhalb der Familie;

für Familien mit recessivgehenden Anomalien:

Kreuzung aller Individuen, am besten natürlich auch nur der

Gesunden, nur nach außen hin, mit den Gliedern eines gesunden

fremden Stammes.

Rufen wir uns alle bisher erwähnten, für die Mendelsche Lehre charakteristischen Punkte noch einmal in Erinnerung, so ist zu erkennen, daß sie zahlreiche Berührungspunkte mit der sog. Mutationstheorie von $H$. de Vries bietet.

Auch sie rechnet mit dem Aufbau der Organismen aus scharf voneinander unterschiedenen Einheiten und ferner damit, daß nicht die äußerlich wahrnehmbaren Merkmale für den Ausfall der Bastarde entscheidend sind, sondern die innerlichen, dem Auge verborgenen Elemen- 
tareigenschaften oder Faktoren, weshalb auch sie zu einer starken Betonung der Diskontinuität im Erbzusammenhang der manifest werdenden Erbeinheiten, also der äußerlich sichtbaren Merkmale gelangt.

Bevor wir weitergehen ist hier der Ort, den Wertmesser im eigentlichsten mathematischen Sinne, welchen wir in der Mendelschen Vererbung kennen gelernt haben, noch an einige andere mathematischen Systeme der Erblich keitsberechnung anzulegen und wirkliche und scheinbare Widersprüche mit ihr aufzuklären.

So lehrt die berühmt gewordene Theorie vom Ahnenerbe Galtons oder das Gesetz von der Mischung elterlicher Eigenschaften in den Kindern, daß die einzelnen Merkmale und Merkmalskomplexe beim Menschen eine rein genealogische Wertigkeit besitzen, d. h. daß die erbliche Übertragung der Merkmale in einer gesetzmäBig fallenden Progression vor sich geht, je nachdem sie dem Vater oder der Mutter, den Großeltern oder noch ferneren Ahnen zukommen. Ein gegebener Proband hat von Vater und Mutter 1/2, von den Großeltern 1/4, d. h. er hat mit den Eltern halbe, mit den Großeltern Viertels-, mit den Urgroßeltern Sechzehntelsverwandtschaft.

Die durch bestimmte Merkmale charakterisierte Erbmasse verdünnt sich also um so mehr, je weiter entfernt verwandt der belastende von dem belasteten Träger eines Merkmals oder Merkmalskomplexes ist.

Setzen wir also nach Galton z. B. den Anteil, welchen beide Eltern zusammen an dem im Kinde zur Entfaltung kommenden Anlagenkomplex besitzen, auf $1 / 2$ (also durchschnittlich die Hälfte), den Anteil der vier Großeltern auf $1 / 4$, denjenigen der acht Urgroßeltern auf $1 / 8$ usw., so läßt sich der Anteil der ganzen Aszendenz an der gesamten Erbmasse des einzelnen Individuums durch die Reihe

darstellen.

$$
1 / 2+1 / 4+1 / 8+1 / 16+\ldots \ldots \ldots \ldots=1
$$

Demgegenüber vertritt Mendels Lehre eine selbständige, und zwar ganz gesetzmäßige, absolute Wertigkeit der einzelnen Merkmale, welche im Prinzip unabhängig sind von der Ausprägung an den Eltern und Voreltern, auch vom Geschlecht des sog. Überträgers. Oder anders ausgedrückt:

Das Prinzip der Regel besteht darin, daß

1. je zwei gewissermaßen konkurrierende Unterscheidungsmerkmale der beiden Eltern sich bei der Vererbung nicht als gleichwertig erweisen (Prävalenzseite), sondern eine typische Ungleichheit im Sinne von Dominanz und von Recessivität erkennen lassen und daß

2. das recessive Merkmal in der zweiten Bastardgeneration wieder durchbricht, daß also, was nach Galto n gar nicht zu erklären ist, in der zweiten Bastardgeneration keine Gleichmäßigkeit der Sprößlinge und keine gleichartige Elternähnlichkeit der Sprößlinge zu konstatieren 
ist, sondern im Gegenteil eine Spaltung in die Merkmale der Großeltern (d. h. getrennt in dasjenige der Großmutter und das des Großvaters, nicht in irgendeine Vereinigung oder Verschmelzung des großmütterlichen und großväterlichen Merkmals) im Verhältnis von 3:1.

Oder noch anders ausgedrückt: Ein gegebener Proband hat vom Vater und der Mutter nicht das halbe, von den Großeltern nicht das Viertelsmerkmal usw., sondern das voll ausgeprägte Merkmal des Vaters oder der Mutter, oder des Großvaters usw., d. h. er ist mit den Verwandtschaftsmitgliedern bezüglich des erblichen Merkmals, das der Betrachtung unterliegt, im einfachen Mendelschen Falle äußerlich völlig verwandt oder gar nicht.

Es sei ferne von mir, damit sagen zu wollen, daß Galtons statistische Beobachtungen und diejenigen der englischen biometrischen Schule (Pearson, Darbishire u. a.) unrichtig, sowie die aus ihnen abgeleiteten „Gesetze" für alle Fälle unzutreffend seien. Gewiß nicht. Sie erklären aber nicht den faktischen Erbgang des einzelnen Merkmals und Merkmalskomplexes durch die Generationen, sie stellen keine Regeln des Vererbungsmechanis mus dar, sie sind keine biologischen Vererbungsgesetze, keine Naturgesetze, sondern nur, wenigstens für manche Fälle (Bateson behauptet auch hier nur für Zufälle) der statistische Ausdruck des schließlichen Endresultats des Wirkens der Mendelschen Gesetze, nur statistische Formulierungen, die für die Massen gültig sind, deren einzelne genetische Einheiten aus dem speziellen genetischen Erbverbande, innerhalb dessen sie in der lebenden Natur eingeordnet sind, losgelöst und zur rein statistischen Operation verwendet wurden, weshalb auch Johannsen sich zu dem Ausspruch berechtigt hielt, die Galto nschen Vererbungsgesetze seien nur der Ausdruck dafür, daß bei den betreffenden Untersuchungen mit unreinem Material gearbeitet worden sei.

Das Galton sche Gesetz ist kein Naturgesetz, sondern nur eine statistische Formel, die höchstens ein Durchschnittsverhältnis richtig zum Ausdruck bringt.

Daß es daher unmöglich ist, auf Grund dieser Formel, Vererbungsintensität und Vererbungsart für ein einzelnes Individuum vorherzusagen, ist klar, weshalb auch in diesem Punkte die Leistungsfähigkeit der Ahnenerbetheorie mit derjenigen der Mendelschen Lehre gar nicht zu vergleichen ist, wenn auch, wie Haecker sehr richtig bemerkt, die Galtonsche Formel bis zu einem gewissen Grade mit den oft allerdings wissenschaftlich recht unklaren und unrichtigen Vorstellungen im Einklang steht, welche sich die Tierzüchter hinsichtlich der „Blutmischung" gebildet haben und die in den üblichen Bezeichnungen $3 / 4$-Blut, $7 / 8$-Blut usw. ihren Ausdruck gefunden haben. 
Auch die Belastungsziffern, wie sie aus Diems Untersuchungen hervorgehen, sind natürlich nur ein mathe matischer Ausdruck für Durchschnittsverhältnisse.

Sollte es sich herausstellen, daß auch die Geistesstörungen oder die Anlagen dazu, wenn sie sich vererben, nach Mendelschen Regeln vererben, so würden wir damit weit über die durch die beste heutige Statistik, nämlich die Die m sche Arbeit bestätigte allgemeine Erfahrung (S. 358 und 359, 1905) emporsteigen, ,daß psychische Krankheit wie Gesundheit sich vererben kann und daß die Gefahr der eintretenden Vererbung nicht unterschätzt werden darf, daß sie vor allem bei geisteskranken Eltern sehr nahe liegt und auch bei geisteskranken Geschwistern ernste Aussichten bietet", daß aber andererseits die Vererbung des Pathologischen doch kein ewiges, unabwendbares Verhängnis ist.

Wer diese, unseren Hoffnungen und Befürchtungen noch so unendlich großen Spielraum belassenden Sätze mit den präzisen unausweichbaren Schlußfolgerungen vergleicht, welche die Mendelsche Lehre zuläßt, wird nur wünschen können, daß vereinte Anstrengung recht bald auf unserem Gebiet die Anwendung jener Regeln gestatten möge.

Lehrt schon ein Blick auf die Fig. 7 und 8, welche auf dem Prinzip der einfachsten Form Mendelscher Recessivität aufgebaut sind, wie sie bei der Vererbung zahlreicher anatomischer und physiologischer, anormaler und normaler, wichtiger und unwichtiger Merkmale bei Tier und Pflanze experimentell festgestellt wurde, in wie mannigfaltigen Proportionen die Regel zutage treten, ihr Walten uns aber auch durch mehrere Generationen hindurch verschlossen sein kann, um plötzlich, mit elementarer Gewalt, in einer Reihe von, wie man dann gewöhnlich sagt, ganz unerwarteten Erkrankungen wieder offenbar zu werden, so kann durch Hinzutreten einer einfachen Komplikation die Mannigfaltigkeit und scheinbare Regellosigkeit des Vererbungsbildes erheblich vergrößert werden.

Diese einfache Komplikation besteht, wenn ein und dieselbe Anomalie nur für die männlichen Glieder der Familie dominant, für die weiblichen dagegen recessiv ist.

Ein Schema derartiger Vererbung gebe ich in Fig. 11.

Die ebenso mannigfaltigen als exakten Belastungs- und Vererbungsberechnungen, die sich an dieses Schema anschließen ließen, kann ich, nach dem bereits Gesagten, dem Leser überlassen. Die „Geschlechts affinität" gewisser Erkrankungen kannte man ja längst (Hämophilie, Farbenblindheit), allein man kannte nicht die Proportionenseite des Phänomens, und wenn einmal vereinzelt ein Glied des anderen Geschlechts krank befunden wurde, sprach man von Ausnahmen, oder auch, man bestritt die Identität dieser Krankheitsform mit der 
echten geschlechtsaffinen Anomalie. Auch hier wieder ein schönes Beispiel dafür, daß „Ausnahmen“ wahrer Wissenschaft fremd sind und nur Regeln „,bestätigen“, die man in Wirklichkeit noch gar nicht kennt.

Allein es gibt noch eine große Anzahl weiterer experimentell auf

\section{Vererbung der Farbenblindheit.}

(Nach Bateson.)

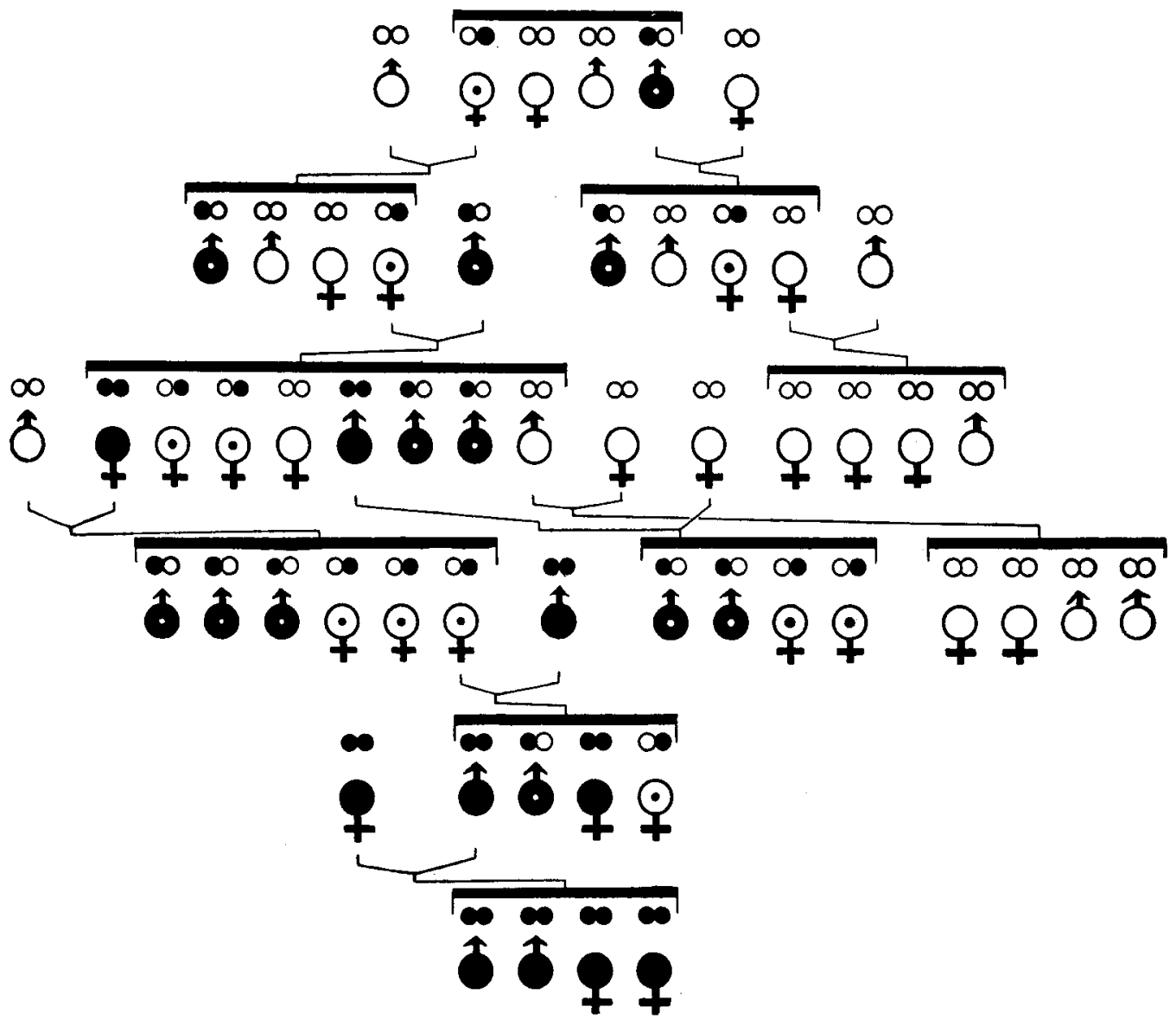

Fig. 11 .

echte Regelmäßigkeiten zurückgeführten Komplikationen des Mendelschen Vererbungsmodus, die der Psychiater als Möglichkeiten wenigstens alle kennen sollte, wenn er den verwickelten, sprunghaften und dunklen und scheinbar oft so launischen Gang der Vererbung auf seinem Gebiet ins helle Licht des Tages bringen und die zahlreichen „Ausnahmen“ auf wahre Regeln zurückführen will. 
Bei der bisherigen Besprechung der Kreuzungsregeln bei Erbsen und beim Menschen wurde angenommen, da $B$ sich die beiden zur Kreuzung zugezogenen Eltern und ihre Stammformen nur in einem Merkmalspaar unterscheiden (rot - nicht rot, d. h. weiß; farbenblind - nicht farbenblind).

Es wurde also angenommen, daß die Nachkommen Monohybride

Kreuzung von Erbsen mit roten Blüten und gelben Kotyledonen mit solchen mit weißen Blüten und grünen Kotyledonen.

(Schema der Anlagen-Kombination.)

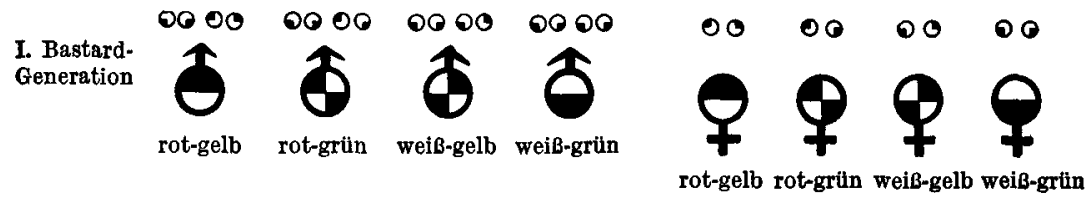

II. Bastard-Generation $0000 \quad 0000 \quad 0000 \quad 0000$
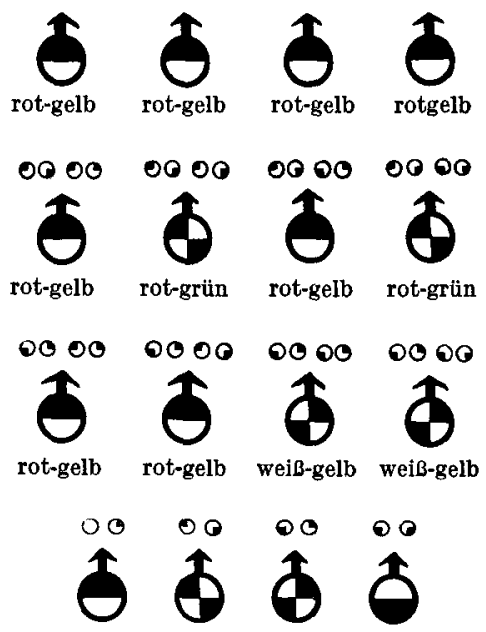

rot-gelb rot-grün weiß-gelb weiß-grün

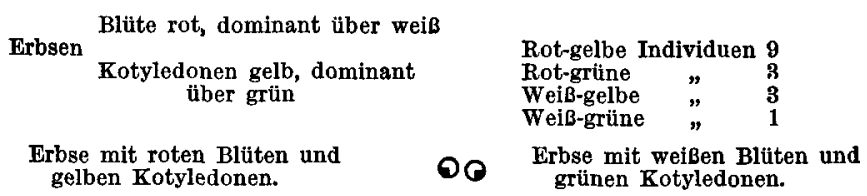

Fig. 12.

sind, d. h. von Eltern abstammen, die tatsächlich nur in dem einen Merkmalspaar differieren. Unterscheiden sich nun aber die beiden miteinander gekreuzten Eltern durch 2, 3 oder mehrere Merkmalspare, sind also die Kreuzungen dihybrid, trihybrid, polyhybrid, so gilt nach Mendel der wichtige Grund- 
satz, daß die einzelnen Merkmalspaare sich mit Bezug auf das Spaltungsphänomen unabhängig voneinander verhalten.

Sind die Eltern z. B. in 2 Merkmalspaaren verschieden, so liefert der Bastard nicht bloß zweierlei Gameten, wie z. B. in Fig. 2, Reihe VI, sondern viererlei Gameten, und durch wechselseitige Vereinigung der männlichen und weiblichen Gameten werden nicht, wie in der monohybriden Kreuzung der Fig. 2, Reihe VII, 4, sondern 16 verschiedene Sorten von Zygoten gebildet. Diese Selbständigkeit im Spalten verschiedener Merkmalspaare bezeichnet man als Unabhängigkeitsregel oder als Regel der Selbständigkeit der Merkmale.

Kreuzt man z. B. (Fig. 12) eine rotblühende Erbsenart, die gelbe Kotyledonen besitzt, mit einer Erbsenart mit weißen Blüten und grünen Kotyledonen, wobei rot über weiß und gelb über grün dominiert, so liefert der Bastard erster Generation, der selbst rot blüht und gelbe Kotyledonen besitzt, im männlichen und weiblichen Geschlecht je viererlei Gameten (Fig. 12), nämlich Gameten mit der Anlage zu rotgelb, rotgrün, weißgelb und weißgrün. Durch Befruchtung, d. h. durch Kombination dieser Gametenanlagen werden dann 16 verschiedene Zygoten gebildet. Doch entstehen daraus, infolge der Dominanz von Rot und Gelb nur viererlei äußerlich verschiedene Bastarde zweiter Generation, und zwar rot(Blüten)-gelbe (Kotyledonen), rot-grüne, weiß-gelbe und weiß-grüne im Zahlenverhältnis von $9: 3: 3: 1$.

Bei trihybriden Kreuzungen, wo die Eltern sich also durch 3 antagonistische Merkmalspaare unterscheiden, würde, wenn wir die dominanten Merkmale mit A, B, C, die dazugehörigen recessiven mit a, b, c bezeichnen, der Bastard erster Generation das Aussehen ABC zeigen, und es würden in der zweiten Bastardgeneration 8 äußerlich unterscheidbare Typen auftreten in folgendem Zahlenverhältnis und mit folgenden Erbformeln:

$$
\begin{gathered}
27 \mathrm{ABC}: 9 \text { aBC: } 9 \mathrm{ABc}: 9 \mathrm{AbC} \\
3 \text { Abc: } 3 \text { aBc }: 4 \text { abC } \\
1 \text { abc. }
\end{gathered}
$$

Die Experimentatoren haben es denn auch wirklich verstanden, durch sorgfältige und große Zuchten, sowohl bei di- als auch trihybriden Kreuzungen, sehr genaue, den theoretischen Werten außerordentlich nahe kommende faktische Zahlenproportionen zu erreichen.

Besonders komplizierend und bedeutsam für die Auffindung von Vererbungsregeln beim Menschen sind die Spaltungen, welche zutage treten bei der Kreuzung einer Stammform mit einer Defektrasse, d. h. mit einer Abart, welche durch das Fehlen eines Merkmals gekennzeichnet ist. Vorgänge, die dazu geführt haben, die einfachere 
Mendelsche Erklärung der Spaltung als einer Trennung von zwei antagonistischen Merkmalen allmählich zu verlassen und sie vielmehr in der Anwesenheit und Abwesenheit eines unterscheidenden Elementes zu suchen (Presence- und Absencetheorie, Faktorenhypothese).

Kreuzt man wildfarbene graue Mäuse oder Kaninchen mit Albinos (also eben einer Defektrasse), so erhält man in der ersten Bastardgeneration lauter graue Bastarde und in der zweiten Bastardgeneration oder Spaltungsgeneration graue und weiße im Verhältnis von $3: 1$.

Allein bei manchen Kreuzungen mit Albinos kommen zuweilen in der Spaltungsgeneration neben den elterlichgefärbten auch schwarze Tiere vor, und zwar in der bestimmten Proportion von 9 grauen: 3 schwarzen : 4 Albinos.

Nach Cuénot kann man das so erklären, daß man 2 mendelnde Merkmalspaare annimmt.

\section{Dominant.}

1. Chromogen (Farbbestimmer) C.

2. Graubestimmer G.
Recessiv Albinismus A. Schwarzbestimmer S (im Albino enthalten).

Es werden also von jedem Geschlecht viererlei Gameten gebildet, nämlich (s. Fig. 13):

$\mathrm{CG}, \mathrm{CS}, \mathrm{AG}$ und $\mathrm{AS}$, und demnach 16 Gruppen von Zygoten. In diesen sind die 4 Faktoren in 9 verschiedenen Kombinationen enthalten (1-9), von welchen 6 (l-6, auf 12 Zygotengruppen verteilt) den Faktor C (Chromogen) aufweisen, also gefärbte (graue oder schwarze) Individuen aus sich hervorgehen lassen, während bei 3 Kombinationen (7-9 in 4 Zygotengruppen) jener Faktor $\mathrm{C}$ fehlt, also weiße Individuen (Albinos) entstehen müssen.

Wie man aus Fig. 13 sieht, haben die 4 Albinos dreierlei gametische Zusammensetzung, also dreierlei Erbwert, und so erklärt es sich denn also in gesetzmäßiger Weise, wenn diese Verschiedenheit der äußerlich gleichen Albinos bei weiterer Kreuzung tatsächlich zutage tritt.

In unserem Falle ist also ein vom Albino (AS $=$ Albino) latent mitgeführtes Merkmal ( $\mathrm{S}=$ schwarz) durch die Kreuzung sichtbar geworden, und zwar dadurch, daß der betreffende positive Faktor (S) mit einem anderen, gleichfalls positiven, im Albino aber nicht enthaltenen Faktor, nämlich dem Chromogen $\mathrm{C}$, zusammengetreten ist, nämlich in allen Erbformeln, die der Farbe Schwarz zugrunde liegen: CSCS; ASCS und CSAS.

Das Gesagte diene nur als ein Beispiel eines gut erdachten, diese Art analytischer Spaltungen etwas befriedigender, als Mendel dies 
zu tun vermochte, erklärenden Deutungsversuches der Entstehung von „Kreuzungsnova“".

Es darf, bei der Verwickeltheit der Phänomene, der Neuheit dieser Forschung und angesichts der Tatsache, daß sogar mehrere Kreuzungsnova durch Kombination der in den Stammformen zutage tretenden

\section{Vererbung des Farbkleides bei Mäusen.} Schema der Anlagenkombinationen.

Eltern :

I. Bestardgeneration. deren viererlei Gameten.
CG

AS (Albino)
II. Bastardgener.
mit 16 Zygotengruppen.
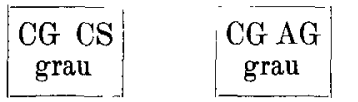

\section{CG AS} grau

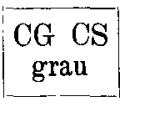

\section{CS CS} schwarz
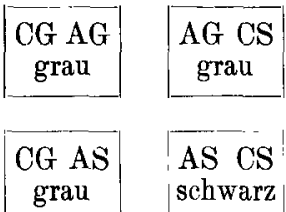

AS CS

schwarz

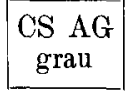

CS AS schwarz

\begin{tabular}{|c|c|}
\hline $\begin{array}{c}\text { AG AG } \\
\text { Albino }\end{array}$ & $\begin{array}{c}\text { AG AS } \\
\text { Albino }\end{array}$ \\
\hline
\end{tabular}

AS AG

AS AS

oder in die 9 Gruppen gleichartiger Zygoten verteilt:

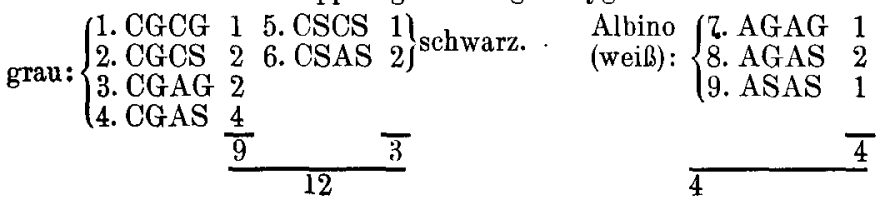

16 Gruppen von Zygoten.

Die Gruppen 1-6, 6 Faktoren- Die Gruppen 7-9, 3 Faktorenkombinationen in 12 Zygoten- kombinationen in 4 Zygotengruppen weisen den Faktor $\mathrm{C}$ gruppen, weisen den Faktor $\mathrm{C}$ (Chromogen) auf, sind also ge- nicht auf, müssen also farblos färbt (grau oder schwarz).

Fig. 13.

und versteckten Faktoren entstehen können, wohl kaum einer besonderen Erwähnung, daß andere Forscher noch andere Darstellungen und Deutungen der Entstehung dieser analytischen Neuheiten gegeben haben. Auch die Erbformeln werden verschieden geschrieben, doch sind als die besten diejenigen zu bezeichnen, aus welchen sofort ersichtlich ist, welche Eigenschaft dominiert und wieviele latente recessive 
Eigenschaften vorhanden sein können, woraus sich dann auch die Zahl der möglichen Gameten und eine sichere Berechnungsmethode der nächsten Generation ergibt (Plate).

Als Beispiele aber dafür, wie sehr man in der Erbbiologie nach anschaulicher Exaktheit strebt, seien hier aus Haecker (S. 284) noch ein paar Erbformeln wiedergegeben, die Castle (verbessert von Lang, Zürich), analog den kettenartig verbundenen chemischen Formeln, im Anschluß an seine Kaninchenkreuzungen, aufgestellt hat.

Danach würde z. B. die Erbformel für das homozygote wildgraue Kaninchen lauten:<smiles>[R17]CBr</smiles>

In dieser besonderen Zusammenstellung der Indices für die einzelnen Faktoren stellt der Faktor C (color), der ganz allgemein die Bildung einer chromogenen Substanz bedingt, eine Art von Kern dar, mit dem alle anderen Faktoren direkt oder indirekt verbunden sind. Es soll dadurch zum Ausdruck kommen, daB ohne die Anwesenheit dieses Faktors C (color) überhaupt keine Farbe zur Entwicklung kommen kann. Die übrigen Faktoren sind: A (Agutifaktor, durch dessen Anwesenheit bei den Meerschweinchen eine ganz bestimmte, durch Anpassung erworbene, die „Wildfarbe“ bedingende Verteilung des Pigments innerhalb der einzelnen Haare hervorgerufen wird), U (Uniformitätsfaktor, bewirkt die gleichmäßige Verteilung des Pigments, also Einfarbigkeit, bei dessen Fehlen Scheckzeichnung auftritt), J (Intensitätsfaktor, bewirkt starke, dichte Pigmentbildung), Y (Yellow, Faktor für Gelb), Br. (Faktor für Braun), B (black, Faktor für Schwarz), sowie $\mathbf{E}$ (Extensionsfaktor, bewirkt gleichmäßige Verteilung des braunen und schwarzen Pigments über die mit gelbem Pigment versehenen Körperpartien; seine Abwesenheit bewirkt Beschränkung von Braun und Schwarz auf Augen und Extremitäten). Die Formel bringt durch die Stellung der Indices im besonderen noch zum Ausdruck, daß B. und $\mathrm{Br}$. bei den Kaninchen nur zur Geltung kommen, wenn schon $\mathrm{Y}$ vorhanden ist, und ferner, daß $\mathrm{E}$ nur auf $\mathrm{B}$ und $\mathrm{Br}$. einwirkt.

Für ein komplett homozygotes, einfarbig schwarzes Tier, bei welchem der Agutifaktor fehlt, würde die Erbformel lauten:

usw.

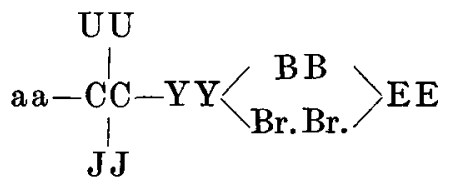


Gemeinsam ist allen Gedankengängen, daß hinter dem äußeren Aussehen eines Individuums noch ein Etwas liegt, was, je nachdem, das Hervortreten neuer, verborgener Eigenschaften bedingt. So ist nach der neuesten Faktorenhy pothese ganz allgemein ein dominierender Charakter durch die Anwesenheit eines bestimmten Faktors (Determinante, Bestimmer, caractère-unité, unit-character, Elementareigenschaft, Erbeinheit, Gen) bedingt, der korrespondierende recessive Charakter durch seine Abwesenheit.

Der positive Faktor wird jetzt in den Erbformeln in der Regel durch einen großen lateinischen Buchstaben, seine Abwesenheit oder der negative Faktor durch den entsprechenden kleinen Buchstaben ausgedrückt, wobei es gewiß richtig ist, mit Plate anzunehmen, daß der recessive Zustand nicht die wirkliche Abwesenheit des betreffenden Faktors bedeutet, sondern vielmehr, daß das Fehlen einer Eigenschaft durch ein bestimmtes materielles Teilchen bedingt wird, während der positive Zustand hervorgerufen wird, indem noch ein neues Körperchen, etwa ein Enzym, hinzutritt (Plate nennt in seiner GrundfaktorSupplement - Theorie jenes Körperchen, welches einem Merkmalspaar zugrunde liegt, „Grundfaktor". Er ist recessiv und aus ihm geht durch Hinzutritt eines „Supplements" der dominante Zustand hervor).

Plate stellt, an Hand der Kreuzung einer schwarzen (s) mit einer weißen (w) Maus, die alte und neue Schreib- bzw. Berechnungsweise zusammen.

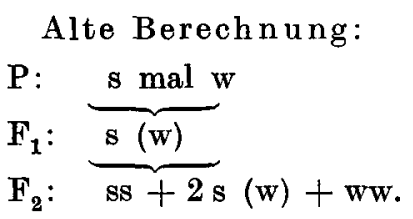

Das Charakteristische ist, daß jede Farbe (schwarz, weiß) als eine besondere Erbeinheit angesehen wird,

Neue Berechnung:

\begin{tabular}{|c|c|}
\hline$P:$ & $\underbrace{\mathrm{CS} \text { mal } \mathrm{eS}}$ \\
\hline $\mathrm{F}_{1}$ : & $\mathrm{Cc}$ SS \\
\hline
\end{tabular}

d. h. jede Farbe gilt als zusammengesetzt aus mehreren Erbeinheiten, was seit $\mathrm{Cuénot}$ schon so sicher gestellt ist, daß man davon als von der „Komplextheorie" sprechen kann, weil ein äußerlich einheitliches Merkmal auf einen Komplex von Erbfaktoren zurückgeführt wird.

Es bildet sich also dann, auf die Mäuse bezogen, ein erstes Paar 
von mendelnden Paarlingen (Antagonisten, Allelomorphen) z. B. C (Anwesenheit von Pigmentfaktor für Pigmentierung) und c (Abwesenheit von Pigment). Ferner der Bestimmer für Grau G, dessen Anwesenheit eine ganz bestimmte Verteilung des Pigments innerhalb der einzelnen Haare, eben die „Wildfarbe" bewirkt und sein Allelomorph (oder Antagonist) g (Abwesenheit des Faktors für Grau); ferner $\mathbf{N}$ (Faktor für Schwarz $=$ noir $=$ nerus) und $\mathrm{n}$ (Abwesenheit des Faktors für Schwarz). Auch schokoladefarbene Mäuse gibt es mit dem Faktor Ch (Faktor für Schokoladenfarbe) und ch (Abwesenheit dieses Faktors). Früher drückte man sich so aus, daB man sagte, $G$ ist dominant über $N$ und $\mathrm{N}$ ist dominant über $\mathrm{Ch}$ oder $\mathrm{G}>\mathrm{N}>\mathrm{Ch}$, wobei $\mathrm{G}$ und $\mathrm{N}$ einerseits, $\mathrm{N}$ und $\mathrm{Ch}$ andererseits als ,korrespondierende" oder allelomorphe oder antagonistische Merkmale gedacht wurden.

Nach der Faktorenhypothese gibt es aber keine Antagonistenpaare von der Zusammensetzung GN, GCh, NCh, sondern nur die Paare Gg, Nn, Chch.

Wenn also z. B. beim Zusammentreffen von $\mathrm{G}$ und $\mathrm{N}$ in einer Zygote die Graufärbung hervortritt, also Schwarz $(\mathrm{N})$ verdrängt oder verdeckt, so sagt man nicht mehr, daß Grau über den Antagonisten $\mathrm{N}$ dominiert, sondern man sagt, er überlagert ihn oder $G$ verhält sich epistatisch gegenüber $\mathrm{N}, \mathrm{N}$ hypostatisch gegenüber $\mathrm{G}, \mathrm{d}$. h. man stellt sich vor, daßder Bestimmer für Grau durch seine Anwesenheit unter anderem auch verhindert, daß der gleichzeitig anwesende Bestimmer für Schwarz seine Wirkung manifestiert.

So erhält man dann, wobei wiederholt sei, daß sich die verschiedenen Autoren über die Einzelheiten mancher Farb-Entstehung und ihrer Erbursachen noch nicht geeinigt haben, die Erbformeln neuer Schreibweise

für graue Mäuse CGNCh. Am besten ist es, dabei Buchstaben zu wählen, welche an die betreffenden Eigenschaften erinnern (wobei G epistatisch gegenüber $\mathrm{N}$ und $\mathrm{Ch}$ ),

für schwarze Mäuse $\mathrm{CgNCh}$ (wobei $\mathrm{N}$ epistatisch gegenüber $\mathrm{Ch}$ ), für chokoladenfarbige Mäuse $\mathrm{CgnCh}$.

Dem Begriff der Dominanz ist also der Begriff der Epistase beigefügt worden, der gestattet, die Verteilung mehrerer Eigenschaften auf die Nachkommenschaft zu berechnen.

Diejenigen von den korrespondierenden Eigenschaften, welche imstande sind, eine andere in der ersten Bastardgeneration völlig zu unterdrücken, nennt man jetzt nicht mehr die dominanten (bzw. die unterdrückten, die recessiven), sondern jetzt werden die Ausdrücke epistatisch bzw. hypostatisch dafür gebraucht, und Dominanz, respektive Rezession wird nur auf diejenigen zusammengehörigen Merkmale an- 
gewandt, welche sich bei der Gametenbildung von einander trennen und in verschiedene Keimzellen desselben Individuums einwandem.

Dieselben Grundsätze gelten für die Erzeugung synthetischer Neuheiten, bei der die Bastarde erster Generation einen neuen, bei keiner der Stammform sichtbaren, speziell einen atavistischen Charakter zeigen, also ein Kreuzungsnovum darstellen (wenn z. B. bei Kreuzung der albinotischen Hausmaus mit der schwarz und weiß gescheckten japanischen Tanzmausrasse ausschließlich graue, wildfarbene Bastarde erster Generation, also Atavismen, Rückschläge auf die Stammform erfolgen).

Jedenfalls halte man fest, daß auch bei diesen Auflösungsund $\mathrm{Zusammenführungsprozessen} \mathrm{von} \mathrm{zusammengesetzten}$ bzw. a useinander gespaltenen Merkmalen die verschiedenen Eigenschaften in der Generationsfolge ganz nach den für die dihybriden (Fig. 12, S. 34) und polyhybriden Kreuzungen geltenden Mendelschen Regeln und Proportionen in die Erscheinung treten.

Die Mendelschen Spaltungen haben sich im Pflanzenreich bei allen gut studierten Pflanzen (Antirrhinum majus, Mirabilis jalappa, Lathyrus odoratus, Hafer, Gerste) erweisen lassen.

Auch sonst findet die Anwendung der Mendelschen Regel auf die Natur durch die Forscher in steigendem Maße statt, wenn auch die Beweise noch nicht überall als stichhaltig angesehen werden können.

Es mendeln anatomische oder morphologische, wie physiologische Charaktere, normale wie abnorme, ganz besonders weitgehend erbliche Rassenmerkmale, wie Färbungs- und Zeichnungscharaktere.

So im Gebiete der Botanik nicht bloß zahllose morphologische Charaktere, Farben, Formen der verschiedenen Organe, deren Aufzählung mir hier erlassen sei, sondern namentlich auch physiologische Merkmale und Dispositionen.

Beim Weizen z. B. Frühreife $>(=$ dominant oder epistatisch über $)$ Spätreife, ebenfalls beim Weizen (nach Biffen): die Rostempfänglichkeit bei gewissen Sorten > Immunität gegen Gelbrost.

Beim Bilsenkraut (Correns), zweijährige Wachstumsperiode von Hyoscyamus niger $>$ einjährig.

Auf dem Gebiete der Zoologie (zumeist bei den domestizierten Tieren) gehen nach Mendel und sind dominant oder epistatisch $(>)$ : Bei Hühnern: der Erbsenkamm und Rosenkamm > einfacher Kamm, enge Nasenlöcher $>$ weite Nasenlöcher, gewöhnlicher Schädel $>$ domartiger Auswuchs bei Houdans.

\footnotetext{
(Schwanzfedern $>$ Fehlen des Schwanzes.

$\{$ Kurzhaarigkeit $>$ Angorismus.

Kaninchen: Rosettencharakter > Glatthaarigkeit.
} 
Bei Mäusen: $\left\{\begin{array}{l}\text { Wildfarbe }>\text { schwarz }>\text { braun }>\text { weiß, } \\ \text { einfarbig }>\text { gescheckt. }\end{array}\right.$

Bei der Gartenschnecke: Einfarbjgkeit $>$ Fünfbänderigkeit.

Bei Axolotl: schwarz > weiß.

Vollblutpferde: braun $>$ fuchsfarbig.

Hornlosigkeit der Aberdeen-, Angus- und Hallowayrinder $>$ Hornbildung.

Ủberhaupt und besonders bei den Nagern: Pigmentierung $>$ Pigmentlosigkeit (Kaninchen, Meerschweinchen, Ratten, Mäusen).

Aber bei Schafen: weiß>schwarz.

Verkrümmung des Schwanzes bei der Katze von Man (unvollkommen) $>$ normaler Schwanz.

Physiologisch: bei Mäusen die normale Bewegung $>$ Tanzen der Tanzmäuse.

Bei Hühnern: der Trieb zum Brüten $>$ schlechtes Brüten.

Bei wildlebenden Tieren: beim Steinkauz (Athene noctua) wahrscheinlich gelbe Iris > Schwarzäugigkeit.

Amsel (Turdus merula): normaler Melanismus $>$ Albinismus.

Pathologisen erbliche Rassencharaktere: Extrazehe der Dorking- und Houdanhühner, unvollkommen > normale Zehenzahl; normaler Kopf $>$ Kopfhernie des polnischen Huhns (Cerebralhernie).

Beim Menschen:

Nach Davenport: welliges und lockiges Kopfhaar $>$ straff; pigmentreicher Zustand der Regenbogenhaut > Pigmentarmut; daher: schwarze Augenfarbe $>$ braune $>$ gelbe $>$ blaue.

Dunkle Farbe der Neger $>$ Albinismus beim Neger.

Pigmentierung tahitischer Polynesier $>$ Pigmentierung der Weißen (Townsend).

Habsburger Unterlippe beim männlichen Geschlechte fast ausschließlich $>$ normale Lippen.

Einfache Geburt wahrscheinlich $>$ Mehrlingsgeburt.

Huntingtonsche Chorea $>$ normal.

Familiäre periodische Paralyse $>$ normal.

Porokeratosis > normal.

Symmetrische Keratodermis der Extremität $>$ normal.

Ủberhaupt familiäre Dyskeratosen $>$ normal.

Distichiasis $>$ normal.

Nachtblindheit $>$ normal.

Ektopia lentis $>$ normal.

Iriskolobom $>$ normal.

Progressive Muskelatrophie $>$ normal.

Hämaturie > normal. 
Spätptosis > normal.

Ptosis familiaris $>$ normal.

Familiäre Hypospadie > normal.

Familiäre hämolytische Cholämien > normal.

Normal > amaurotische Idiotie.

Manche familiäre Muskelleiden $>$ normal.

Familiäre Psoriasis $>$ normal.

Familiäre Neurofibromatosis $>$ normal.

Cerebellare Heredoataxie $>$ normal.

Manche Formen von Ichthyosis palmaris $>$ normal.

Multiple osteogene Exostosen $>$ normal.

Multiple Teleangiektasie $>$ normal.

Normal > Albinismus.

Brachydaktylie oder Hypophalangie > normal.

Kongenitaler grauer Star $>$ normal.

Eine ganze Anzahl von Hautaffektionen > normale Haut.

Z. B. Tylosis palmaris et plantares $>$ normal.

Dermatolysis hereditaria (Epidermolysis bullosa) $>$ normal.

Hypotrichosis congenita familiaris $>$ normal.

Monilithrix > normal.

Diabetes insipidus (Polyurie) $>$ normal.

Kongenitale stationäre Nachtblindheit $>$ normal.

Einige Formen von Glaukom $>$ normal.

Normal $>$ Retinitis pigmentosa (aber auch in anderen Familien andere Formen?).

Normal > Alkaptonurie.

Farbenblindheit bei Männern $>$ normal.

Hämophilie bei Männern > normal.

Paralysis muscularis pseudohypertrophica (Gowers) $>$ normal.

Essentieller Sehnervenschwund bei Männern $>$ normal.

$\mathrm{Ob}$ die Mendelschen Spaltungen nun aber unumschränkte Gültigkeit für das organische Reich besitzen, ist noch eine offene Streitfrage.

Der Vollständigkeit halber sei erwähnt, daß die Mehrzahl der Zoologen noch am Bestehen einer zweiten Vererbungsart, an der intermediären oder verschmelzenden Vererbung festhält, bei welcher die korrespondierenden Eigenschaften der Eltern in den Kindern der ersten Bastardgeneration als ein Mischprodukt zum Vorschein kommen.

Als Beispiele, deren Beweiskraft aber vielfach auch bestritten wird (in Ansehung der äußeren Erscheinung der späteren Bastardgenerationen.) sind zu nennen: die Mulattenfarbe, Mischung aus Negerschwarz und Weiß, die mittlere Ohrlänge der Kaninchen als Kreuzungsresultat 
langohriger und kurzohriger Eltern. Mit einer in der tatsächlichen Beobachtung nicht immer begründeten Sicherheit wurde bisher auch beim Menschen in der Pathologie allgemein mit dieser Art der Vererbung gerechnet, indem angenommen wurde, daß irgendein Erbmakel durch Mischung oder Verdünnung mit guten Erbmassen gemildert und schließlich beseitigt werden könne.

Der intermediäre Charakter gewisser Vererbungsvorgänge soll hier nicht in Abrede gestellt werden. Es frägt sich aber einerseits, ob er dem Mendelschen Typus prinzipiell gegenüber zu stellen ist. Seine Kombination, wenn ich mich so ausdrücken darf, mit der Mendelschen Vererbung im Zeatypus (s. oben S. 496) muß uns in dieser Richtung vorsichtig machen. Andererseits verschafft uns gerade der Umstand, daß manches, was früher als intermediär sich vererbend galt, später auf Mendelsche Vorgänge zurückgeführt werden konnte (Lang, Zürich), die Lehre, daß der Geltungsbereich dieser Vererbungsart jedenfalls kleiner ist, als man denkt.

Nur wenn im Gegensatz zum Zeatypus eine Spaltung auch in der dritten, vierten und den folgenden Bastardgenerationen nicht einträte, würde echte, dauernde intermediäre Vererbung und damit ein als eigenartig zu bezeichnender Vererbungsmodus vorliegen.

Das, was man als neomorphe Vererbung bezeichnet hat, z. B. wenn bei Hühnern aus Rosenkamm mal Erbsenkamm etwas Neues, der Walnußkamm entsteht, welche Neubildung in der zweiten Bastardgeneration $\left(\mathrm{F}_{2}\right)$ aber wieder nach Mendelschen Proportionen spaltet, ist ganz offenbar nur eine Abart Mendelscher Vererbung und rechtfertigt nicht ihre Aufstellung als eigenartigen Vererbungsmodus.

Dasselbe gilt wohl von der Mosaikvererbung, bei der in der ersten Bastardgeneration die elterlichen Anlagen sich innig mengen und über den ganzen Körper oder ein Organ verteilen, aber doch deutlich erkennbar bleiben (z. B. Sprenkelung, Sperberung der Nachkommen). Denn auch hier sind vielfach spätere Spaltungen nach Mendel beobachtet worden.

Die heikle Frage, ob auch auf dem Gebiete der Psychiatrie jetzt schon Störungen bekannt sind, von denen man mit Sicherheit behaupten könnte, sie folgen Mendelscher Regelmäßigkeit, muß ich verneinen. Im Ernste und mit tauglichen Mitteln ist a ber auch, soweit mir bekannt, noch nicht danach gesucht worden. Auch wird der Beweis, da uns das Experiment fehlt, nur sehr langsam und mühevoll zu erbringen sëin.

Heron hat, wie mir scheint, etwas voreilig und mit gänzlich unbrauchbarer Methode geschlossen, daß ,Geisteskrankheit“" nicht mendelt, weder dominant, noch recessiv gehe.

Und Cannon und Rosanoff fanden das Gegenteil, nämlich ein 
recessives Verhalten der Geistesstörungen, wobei die verschiedensten Formen sich gegenseitig zu vertreten imstande seien.

Wie ich später an Hand eines eigenen Materials zeigen möchte, spricht sehr vieles dafür, daß gewisse Formen der De mentia praecox in gewissen Familien dem recessiven Vererbungstypus folgen, und wer die Schemata auf S. 498 und 499 ansieht, wird gewiß unter seinem Erfahrungsmaterial die eine oder andere Familie finden, wo außerordentlich ähnliche Verhältnisse zu finden sind. Je gewissenhafter man sammeln wird (auch bei Gesunden), je fruchtbarer die gesammelten $\mathrm{Fa}$ milien sind, jo seßhafter sie sind, (Leichtigkeit der Untersuchung aller Glieder), je geringer die Mortalität in der Familie ist und je genauere authentische Schilderungen über die Art der einzelnen vorgekommenen Geistesstörungen vorhanden sind, um so eher wird diese Frage einer Lösung zuzuführen sein.

Umgekehrt scheint mir bei manchen manisch-depressiven Störungen in manchen Familien der Gedanke einer dominanten Vererbungsweise nahe zu liegen, denn direkte Vererbung ist hier sehr viel häufiger, wie bei Dementia praecox. Dasselbe gilt von den Geschwisterpsychosen.

Die Dementia praecox ist ihrer Natur nach (unheilbare oder mit Defekt heilbare Früherkrankung) auf eine kollaterale (recessive) Übertragungsweise gewissermaßen angewiesen, wenn man nicht ihr ungeheuer häufiges Vorkommen ausschließlich auf beständige neuschöpfende Variation schieben will.

Die Manisch-Depressiven sind aber in ihrer überwiegenden Mehrzahl im Fortpflanzungsgeschäft durch ihre Krankheit nicht entfernt in so eingreifender Weise behindert, was eben im Zusammenhalte mit den genannten Unterschieden gut mit der Arbeitsannahme einer dominanten Übertragungsweise zumindest mancher Formen in manchen Familien übereinstimmt.

Auch manche Psychopathien, Entartungs- und Defektzustände scheinen sich in dieser Weise zu vererben.

Doch wird sich hierüber Sicheres erst nach Übersicht über ein größeres, klinisch gut beobachtetes Material geben lassen, das auch hinsichtlich jener den reinen Erbgang störenden Außenmomente gut bearbeitet ist, welche ich S. $561 \mathrm{ff}$. bespreche und deren Beachtung bei dem vergiftenden Leben, das der Kulturmensch führt und bei den schädigenden Einflüssen, die von seinen zahlreichen, schwerkranken Körperorganen auf den Keim ausgehen, im Gegensatz zur naturgemäßen Lebensweise und durch natürliche Zuchtwahl hocherhaltenen Allgemeinkonstitution des wildlebenden Tieres, nicht genug empfohlen werden kann.

Die inter mediäre Vererbung, also die innige Verschmelzung der 
zwei psychotischen oder psychopathischen Eigenschaften des Vaters und der Mutter zu einer Mischeigenschaft, die in der Mitte steht, im Nachkommen, ist, nach dem Augenschein zu urteilen, gerade auf dem Gebiete der Psychiatrie wohl verhältnismäßig selten.

Im Gegenteil, es läßt sich in psychotischer oder psychopathischer Beziehung zumeist die Ähnlichkeit einer Probandenabnormität mit der eines ganz bestimmten Familienmitgliedes nachweisen oder aber gewisse Eigenschaften verschiedener Verwandter kommen nebeneinander vor, wobei dann stets die korrespondierenden des anderen elterlichen Geschlechts gewissermaßen unterdrückt sind.

Schließlich hat die Frage der Korrelationen durch die experimentelle Vererbungsforschung bereits eine exakte, von ihrer gewöhnlichen mathematischen Bearbeitung himmelweit verschiedene Behandlung und Beleuchtung erfahren und wird fortan noch viel mehr von ihr zu erwarten haben.

Von Korrelation spricht man, wenn zwei Merkmale derart im $\mathrm{Zu}$ sammenhange stehen, daß sie in ihrem Auftreten gegenseitig aneinander gebunden sind und da $B$ Abänderungen des einen auch solche des anderen mit sich bringen. Z. B. wenn Krankheiten, wie die Bluterkrankheit, mit dem männlichen Geschlecht innig verknüpft sind oder mit bestimmten Gewebsfarben, z. B. des tierischen Haarkleides. So entstehen z. B. bei weißen oder weißgefleckten Schafen und Schweinen, wenn sie mit Buchweizen gefüttert und dem Sonnenlicht ausgesetzt werden, bestimmte, als Fagopyrismus bezeichnete Hautaffektionen, welche bei schwarzen Tieren unter gleichen Umständen nicht hervorgerufen werden.

Dabei sollte von Korrelation im strengen Sinne nicht gesprochen werden, wenn die betreffenden Eigenschaften nur ein verschiedener Ausdruck desselben Zustandes sind (also z. B. Größerwerden von Organen und Zunahme [Caeteris paribus] ihres Gewichtes) oder wenn sie z. B. nur verschiedene Auswirkungen einer Ursache an verschiedenen Organen sind (z. B. Veränderungen verschiedener Natur an verschiedenen Organen durch Alkoholmißbrauch), wenngleich auch die Kenntnis dieser Abhängigkeiten für praktische Rückschlüsse auf eventuelle spätere psychische Erkrankung und die Zeugungsprognose von der allergrößten Wichtigkeit werden kann. Sondern man hat dabei bestimmte Veränderungen eines Organs, Systems oder Gewebes im Auge, die mit einer großen Regelmäßigkeit eintreten, wenn bestimmte andere Organe, Systeme oder Gewebe sich in bestimmter Richtung verändern.

Bekannt ist ja die Abhängigkeit der sekundären Geschlechtscharaktere von den Geschlechtsdrüsen.

Allein es sind Tatsachen zutage gefördert worden, welche beweisen, daß Merkmale, die für gewöhnlich in der ausgeprägtesten physiologi- 
schen Abhängigkeit voneinander stehen (wie die sekundären Geschlechtsmerkmale von den Geschlechtsdrüsen), doch auch ganz losgelöst und anscheinend unabhängig voneinander ihr Dasein führen können ( $\mathrm{Zu}-$ sammenvorkommen von rein männlichen sekundären Geschlechtsmerkmalen mit Eierstöcken usw.), weshalb man auf den Gedanken gekommen ist, daß das geschilderte Verhalten möglicherweise auf verschiedenartige Kombination bestimmter Erbeinheiten im Keime zurückzuführen ist, welche dann auch die verschiedenartigen körperlichen Auswirkungen verständlich machen könnte.

Es ist denkbar (Plate), daß Erbeinheiten vorhanden sind und sein müssen, damit ein äußeres Merkmal direkt hervorgerufen werde. Man kann sie mit Plate „Erregungsfaktoren“ oder Erregungsdeterminanten nennen, im Gegensatz zu den „Konditio nalf a k tore n“", welche vorhanden sein müssen, damit ein Erregungsdeterminant überhaupt wirken kann. Dabei können von einer Erbeinheit mehrere äußere Merkmale abhängen, die Erbeinheit kann also pleiotrop sein, und es treten dann die Merkmale natürlich stets zusammen auf, erscheinen miteinander korrelativ verbunden.

Es scheint aber, daß eine Erbeinheit sogar gleichzeitig für ein bestimmtes Merkmal Erregungs-, für ein anderes Merkmal dagegen Konditionalfaktor sein kann. So schwindet nach Plates Erfahrungen aus der Mäusezucht die Tanzbewegung aus einer einfarbigen Mäuserasse sehr bald wieder, wenn sie durch Kreuzung mit einer gescheckten Tanzmaus in sie hineingekommen ist, während die letzteren rein züchten, wenigstens in vielen Familien. Der Faktor für Scheckung scheint also hier die Vorbedingung für eine konstante Übertragung dieses Merkmals (des Tanzens) zu sein.

Wir dürfen mit solchen Erwägungen, die von bestimmten und der gesetzmäßigen Betrachtung zum Teil schon zugänglichen experimentellen Ergebnissen ausgegangen sind, wohl auch den Begriff der Disposition in Zusammenhang bringen, den man ja in der Pathologie längst vor Mendel postuliert hat und der in der neuen Fassung auf Erbeinheiten zurückzuführen sein würde, die vorhanden sein müssen, damit gewisse andere Erbeinheiten oder auch bestimmte äußere Einflüsse einen Krankheitszustand auslösen können. Und dies zwar in einer im Mendelschen (natürlich komplizierteren) Sinne regelmäßigen Weise. So meint Plate, daß, in der Sprache der Erblichkeitsforschung, die bei den gelben Mäusen, welche besonders zur Unfruchtbarkeit neigen, in den Erbformeln vorhandenen Erbfaktoren yGB oder yGb für die Unfruchtbarkeit mit verantwortlich zu machen seien, und der Erbfaktor $\mathrm{y}$ bedinge die Disposition zum „Schwanzknick" mancher seiner Mäuse.

Den Korrelationen infolge pleiotroper Erregungsfaktoren und in- 
folge pleiotroper Konditionalfaktoren kann man (Bateson) noch die Korrelationen durch unechte Allelomorphie beifügen.

Wie wir sahen (S. 491, Absatz 3), nahm Mendel an, daß die beiden Bestandteile (die dominante und die dazu gehörige recessive Anlage) des mendelnden (allelomorphen) Merkmalpaares bei der Bildung der Geschlechtszellen in verschiedene Geschlechtszellen einwandern. Dominant stößt gewissermaßen recessiv ab.

Bestehen nun aber in einem Individuum zwei allelomorphe Paare und stoßen sich nicht bloß dominant und recessiv ab, sondern auch dominant und dominant, so wandern die beiden verschiedenen dominanten Eigenschaften ebenfalls stets in verschiedene Geschlechtszellen und es entstehen nicht vier Sorten von Geschlechtszellen, sondern nur zwei. Es entstehen dann nur zweierlei Keime, h. h. solche mit den Anlagen $\mathrm{aB}$ und solche mit den Anlagen Ab, wenn A und $\mathrm{B}$ die beiden dominanten, $\mathbf{a}$ und $\mathbf{b}$ die beiden recessiven Merkmale bedeuten.

Es verhalten sich in diesen Fällen also zwei dominante Merkmale zueinander ebenso, wie sonst die dominante Eigenschaft zu der zugehörigen recessiven.

Die Folge dieser Erscheinung ist eine Beschränkung der sonst im einfachen Falle möglichen Kombinationen der Erbeinheiten.

So fand Bateson (nach Plate) bei Lathyrus odoratus zwei verschiedene Merkmale der Blütenform an die verschiedenen Erbfaktoren blau und rot gekoppelt. Es kommen nämlich Rassen vor, bei welchen das hinterste, größte Blütenblatt, die Fahne, durch seitliche Einrollung eine gebogene „Haube" bildet, während es bei der gewöhnlichen Form einfach und aufrecht ist. Aufrecht dominiert über Haube und blau über rot. Bei einigen Schlägen (nicht bei allen) findet sich nun eine gesetzmäßige Korrelation, indem die Haube immer geknüpft ist an blau und aufrecht an rot. Die beiden dominanten Erbeinheiten aufrecht und blau scheinen also nicht zueinander zu passen; sie wandern nie in dieselbe Gamete. Daher werden von den Bastarden aus jenen Schlägen nicht vier verschiedene Gameten gebildet, sondern nur zwei: die eine erhält aufrecht + rot, die andere: Haube + rot.

Das Experiment bestätigte die aus diesen theoretischen Voraussetzungen berechnete numerische Einschränkung der Kreuzungsprodukte.

Hier liegen also, wie man sieht, schon hervorragende Gesetzmäßigkeiten korrelativer Verknüpfung vor, wenn man auch, wie gleich bemerkt sei, noch längst nicht überall Regeln zu finden in der Lage war, wo man solche stark vermutet. Aber man ist doch auf dem besten Wege dazu, und wer die einschlägige Literatur durchgeht, wird das Gefühl ehrfurchtsvoller Erwartung nicht los, welches auf die 
Erklärungsmöglichkeiten gerichtet ist, die aus der Anwendung dieser mathematischen Methode im Pflanzen- und Tierreich auch für die korrelativen Verknüpfungen der normalen und abnormen Eigenschaften beim Menschen dereinst sich ergeben werden.

Wie man sieht, eröffnet die Befruchtung der Korrelationsund Dispositionslehre (ich erinnere auch nochmals an die Mendelschen Regeln folgende Vererbung der Disposition des Weizens zu Rosterkrankung) durch Mendels Lehre überaus hoffnungsvolle Perspektiven für menschliche Verhältnisse, speziell auch für die Psychiatrie. Sie lehrt uns Regelmäßigkeiten, ja verspricht uns Gesetzmäßigkeiten zu erkennen, wo wir bisher nur von Möglichkeiten, höchstens Wahrscheinlichkeiten sprechen durften.

Ganz besonders die Ps ychiatrie wird sich auf diese Seite Mendelscher Forschung werfen müssen. Denn auf einem Gebiete, wo Anlagestörungen so oft erst später im Leben zum Ausbruch kommen und nur selten in ihren Anfängen schon ganz frühe erkannt und vorausgesagt $\mathrm{zu}$ werden vermögen, muß man besonders dankbar sein für Symptome physischer oder psychischer Natur, welche erweislich in fester Korrelation zu späterer, determinierter Geistesstörung stehen und schon verhältnismäßig früh exakt konstatiert werden können.

So muß daran gedacht werden, da $\beta$ in bestimmten Familien bestimmte Psychosen besonders gern bei Individuen vorkommen, welche auch bezüglich anderer, zum Teil exakt meßbarer Eigenschaften ihres Körpers (anthropologische Merkmale, Asymmetrien, vasomotorische Eigentümlichkeiten, Maßverhältnisse an den verschiedenen Organen, besonders den Augen, dactyloskopische Korrelationen, Lungenkapazität, bestimmte Chemismen, besonders aber meßbare psycho-physische Eigenschaften usw.) in bestimmter Weise sich von jenen anderen der Familie unterscheiden, welche nie erkranken werden.

$\mathrm{Da} ß$ hier zahlreiche Abhängigkeiten bestehen - ich verweise nur kurz auf die Ergebnisse der ausgedehnten Massenuntersuchungen an Schulkindern über die korrelativen Beziehungen von Körper- und Geistesentwicklung, an Schwachsinnigen über korrelative Abhängigkeit des Schwachsinnsgrades vom Grad der Anomalien am Auge u. dgl. - , ist nicht der mindeste Zweifel. Es gilt nur, sie über die Bedeutung von Durchschnittswerten und von Wahrscheinlichkeitsergebnissen hinüberzuheben zu einer Regelmäßigkeit im Sinne der Mendelschen Forschung.

Es wird eine Hauptaufgabe der biologischen Psychiatrie sein, diese korrelativen Merkmale, besonders der präpsychotischen Persönlichkeit (welche begrifflich zu unterscheiden sind von den eigentlichen Anfängen der Psychose), welche den späteren Ausbruch der noch verdeckten Psychose gewissermaßen ankündigen, sowohl für 
die Träger bestimmter klinischer Entitäten überhaupt (allge meingültige Korrelationen), als auch für bestimmte Krankheitsentitäten innerhalb bestimmter Familien und Stämme (f a miliäre Korre. lationen) festzustellen, um sie eventuell als Leitstern zu benützen bei der Eruierung schlummernder geistiger Abnormitäten.

Denn können auch, wie wir gesehen, die einzelnen Merkmale getrennte und sich mannigfach kreuzende Vererbungswege gehen, so ist es doch auffallend, wie angesichts der zahllosen möglichen Kombinationen, die nach einer reinen Wahrscheinlichkeitsrechnung auf Grund der Ahnenerbsumme ausgedacht werden können, in Wirklichkeit doch manche Eigenschaften mit bestimmten anderen konservativ zusammengekoppelt sind und aneinander gebunden immer wiedererscheinen, so daß mit einer gewissen Sicherheit (und eben die Zukunft soll uns Mendelsche Sicherheit bringen) Caeteris paribus aus dem Vorhandensein der einen auf das Vorhandensein oder das spätere Erscheinen auch der anderen geschlossen werden kann.

Einstweilen sind wir von einer sicheren Verwertung korrelativer Verknüpfungen, also auch des Korrelationsbruches, noch weit entfernt.

Erklärungsversuche, wie sie Cox (Direktor der Irrenanstalt in Utrecht) vorgenommen hat (Psychiatrische und neurologische Bladen, 1907, Nr. 1), dürfen nur als vorläufige (und zum Teil unsicher und unzutreffend) bezeichnet werden.

Er faßt die Entartung als Korrelationsbruch auf, als Verlust der Wechselbeziehungen von Eigenschaften, die miteinander in Wechselbeziehung stehen müssen, also ohne Nachteil für das Individuum nicht getrennt werden können. Durch Rassenkreuzung werden viele dieser notwendigen Beziehungen nach Cox gestört, weil die Anlagen in den Keimzellen abweichender Rassen nicht alle zusammenpassen. So entstehen Mißverhältnisse, Lü̈ken, die im Erwachsenen als Disharmonien, Gleichgewichtsstörungen, geistige Minderwertigkeiten hervortreten. Für Mischlinge stark verschiedener Rassen (Mulatten) dürfte der Nachweis, daß dem so ist, in der Tat nicht allzuschwer fallen. Schwieriger liegen die Dinge bei Mischlingen nah verwandter Rassen (z. B. zwischen Homo europäus und Homo alpinus). Doch dürfte auch hier ein Arbeiten mit exakten Methoden der Anthropologie und Vererbungsbiologie zur Aufdeckung pathologisch wichtiger Beziehungen zwischen Korrelationsbruch und krankhafter Veranlagung in dieser oder jener Richtung führen, die im Zusammenhang mit unserem Spezialproblem der Zeugungsprognosebestimmung natürlich um so brauchbarere Ergebnisse liefern wird, je mehr die gewöhnlichen Massenuntersuchungen durch systematische Durchuntersuchung sämtlicher Glieder von ganzen Familien und von ganzen Stämmen ergänzt werden. 
Es darf nicht verschwiegen werden, daß die experimentelle Vererbungsforschung, die fortwährend neue und zum Teil überraschende Tatsachen zutage fördert, noch in gewaltiger, fruchtbarer, schöpferischer Umwälzung begriffen ist und Resultate zutage gefördert hat, deren Regelmäßigkeit und gesetzmäßige ursächliche Bedingtheit noch nicht wie in den oben berichteten Fällen feststeht, ja, die vorläufig noch, rein als Tatsachen genommen, mit den genannten, als eherne Gesetzesnotwendigkeiten imponierenden ursächlichen Verkettungen in einem gewissen Widerspruch zu stehen scheinen.

So ist von vereinzelten Experimentatoren ein gewisser ,Do min a nzwechsel" festgestellt und als Prinzip in die Vererbungsbiologie eingeführt worden, und es scheinen immer mehr Beispiele bekannt zu werden, welche in gewissem Umfange unter gewissen Bedingungen für das Walten eines solchen Prinzipes sprechen.

Es ist darunter zu verstehen, daß ein dominantes Merkmal unter Umständen plötzlich recessiv, oder ein recessives plötzlich dominant werden kann.

Solche vereinzelten Beobachtungen hat auch Plate an seinen Mäusezuchten gemacht. Vielleicht liegt auch in den von Häcker gezüchteten weißen Axolotln, welche später pigmentiert wurden, ein solcher Fall vor.

Plate nimmt auch den Fall der Biene dafür in Anspruch, um die Entstehung der Geschlechter mit dem Mendelschen Schema in Einklang bringen zu können.

Ein Dominanzwechsel sei ferner, meint er, anzunehmen, wenn bei weiblichen Tieren im Alter plötzlich männliche Charaktere hervorbrechen (Bart alter Frauen, hahnenfedrige Hennen, gehörnte Ricken u. dgl.).

Vielleicht kann sich der Dominanzwechsel, und dieser Sachverhalt würde die genaueste Abgrenzung z. B. gegen die später zu besprechenden, als Neuschöpfungen aufgefaßten Greisen- oder Jünglingszeugungen (S. 555) erheischen, auch so äußern, daß die von denselben Eltern in verschiedenen Altersabschnitten erzeugten Jungen verschieden ausfallen.

So erwähnt von Buttel-Reepen, daß eine gelbe italienische Bienenkönigin, welche von einer dunklen deutschen Drohne befruchtet war, im ersten Jahr zahlreiche Mischlinge produzierte, im zweiten fast rein italienische, im dritten ausschließlich italienische Arbeiterinnen lieferte. Die dunkle deutsche Biene wäre nach Mendel als dominant, die italienische als recessiv zu bezeichnen. Es wären dann bei Anwendung des Dominanzwechsels auf die Variantenfolge die ersten Formen als dominante Heterozygoten (Dominant-recessive oder DR-Formen) aufzufassen, wobei später im allelomorphen Paar D (dominant) zu 
$R$ (recessiv) geworden und das allelomorphe Paar RR (homozygot recessiv) entstanden wäre, dem das äußere italienische Aussehen der Biene entsprechen würde.

Plate fügt noch eine Beobachtung Langs bei, wonach bei Kreuzungen von gelben Helix hortensis mit roten Helix nemoralis in seltenen Fällen das recessive Gelb zuerst dominierte und erst im späteren Alter von Rot verdrängt wurde und meint, daß alle diese Beispiele dazu berechtigen, das Prinzip des Dominanzwechsels als Hypothese aufzustellen, zumal eine solche Änderung nach der Grundfaktor-Supplementtheorie nicht schwer zu verstehen sei.

Auch die Theorie der Gametenreinheit glaubte und glaubt man vielfach noch durch diese oder jene Beobachtung erschüttern zu können, denn es ist zweifellos, daß sie mit manchen experimentellen Ergebnissen, z. B. den Beobachtungen beim Axolotl (Häcker, S. 231) zurzeit noch nicht gut in Einklang zu bringen ist.

Man hat sich zur Erklärung dafür, daß die Gameten nach der Spaltung in Wirklichkeit nicht vollkommen rein sind, dabei vorgestellt, daß die bei der Kreuzung vereinigten Anlagen sich gegenseitig beeinflussen können, ehe sie bei der Keimzellenbildung der Bastarde erster Generation wieder auseinander gehen (Tauschhypothese) oder im Bilde nach Castle: die Trennung der Anlagen sei nicht so vollständig, wie wenn man zwei aufeinandergelegte verschiedenfarbige Glasplatten voneinander nimmt, sondern wie wenn man zwei verschieden gefärbte zusammengeschmolzene Wachsschichten trennt.

Oder man hat sich vorgestellt (Alternationshypothese), daß bei der Keimzellenbildung der Bastarde erster Generation überhaupt keine Spaltung der beiden Anlagen im Sinne Mendels stattfinde, sondern daß vielmehr sämtliche Keimzellen beide Anlagen, jedoch mit wechselnder Dominanz, in sich einschließen. Und schließlich fübrt eine dritte Hypothese, die aber in der allgemeinen Form, in der sie vertreten ist (Tschermack), mit Mendels Lehre durchaus vereinbar ist, die vielfach beobachtete Unreinheit der Kreuzungsprodukte darauf zurück, daß die scheinbar rein recessiven Individuen das dominierende Merkmal in „kryptomerem", „latentem" Zustande mit sich führen und daß diese latenten Anlagen durch die Kreuzung zum Teil wieder geweckt werden können.

Allein der Umfang der Tatsachen, welche mit der ursprünglichen Auffassung in scheinbarem Widerspruch stehen, ist doch wohl viel geringer, als man annimmt. Denn es ist bezeichnend, daß im Anschluß an $\mathrm{Cu}$ énot, Morgan und Castle zwar immer die Mäuserassen zur Stütze der Theorie der Gametenunreinheit herangezogen werden, daß aber z. B. Plate gerade bei seinen komplizierten zahlreichen Mäusekreuzungen auf dem Gebiete der Farbenvererbung kein Fall vorgekom- 
men ist, weleher von der Mendelschen Auffassung einer vollständigen, reinen Spaltung antagonistischer Faktoren abwiche.

Man sollte sie daher ohne Not nicht aufgeben und keinesfalls, bevor man auf Grund einer eventuell neuen Auffassung andere Gesetzmäßigkeiten nachzuweisen in der Lage ist.

Der Weg, den die Psychiatrie wird beschreiten müssen, um zu der exakten Beantwortung der Frage zu gelangen, ob die geistigen Anomalien beim Menschen ebenfalls den durch das Tier- und Pflanzenexperiment zutage geförderten einzelnen exakten Vererbungsregeln gehorchen, ist, wie gesagt, fast ausschließlich die systematische Familienforschung, wenn auch das nach psychiatrischen Fragestellungen angeordnete Tierexperiment beim weitschauenden Psychiater nicht wird vernachlässigt werden dürfen. Die durch viele Generationen systematisch betriebene Familienforschung ist beim Menschen ein fast vollgültiger, wenn auch weit langsamer zum Ziele führender Ersatz.

Die Familienforschung hat auf dem durch die experimentelle Vererbungsforschung vorgeschriebenen Weg zunächst die erste Aufgabe, alle Blutsverwandten eines Probanden, dessen Erbformel bestimmt werden soll, nach möglichst zahlreichen naturwissenschaftlichen Gesichtspunkten zu registrieren, sowohl seine Aszendenten, wie auch seine Deszendenten und die Kollateralen, die kranken Verwandten nicht bloß, sondern ebenso die Gesunden, nicht bloß die Geisteskranken, Psychopathen, Nervösen und Nervenkranken, sondern auch die körperlich Kranken oder krankhaft Veranlagten und Defekten, nicht bloß die männlichen Ahnen und Kollateralen, sondern auch die Blutsverwandten der angeheirateten Verwandten. Kurz, auf die Seite der Aufgabe bezogen, welche die Familienforschung im Vererbungsphänomen zu lösen hat:

Sie hat nach oben, nach der Seite und nach unten hin sämtliche Glieder der engeren und weiteren Familie zu registrieren, weil jedes Glied ein ganz bestimmter Erbträger ist, dessen Erbqualitäten ihm aber weder immer anzusehen sind, noch immer in der von ihm erzeugten Generation oder mit einem Partner allein zum Vorschein kommen, weshalb die Anlage weitausgedehnter Verbände von Blutsverwandten nach Analogie der Zuchten der Experimentatoren ein unverläßliches Postulat ist.

Dabei wird natürlich das Material, um Schlüsse zuzulassen, weit größer sein müssen als das der Experimentatoren, worauf ich noch zu sprechen komme.

Sind alle Vorbedingungen erfüllt, so muß es sich bald herausstellen, ob für eine gegebene psychiatrische Anomalie Mendelsche Vererbung 
dieser oder jener Art vorliegt oder nicht, welche Spezialfrage für das Gebiet der Vererbung zu lösen zunächst die wichtigste Aufgabe des Psychiaters ist.

Bei der Materialbeschaffung ist der Grundsatz zu verfolgen, möglichst viele kranke wie gesunde Blutsverwandte des in Betracht fallenden Probanden persönlich zu sehen, über sich selbst und über die anderen Verwandten zu befragen und sie, wenn nötig und möglich, zu untersuchen.

Aus dem gesammelten Material wird zweckmäßig, zur Ưbersicht über die Verwandtschaftsbeziehungen der einzelnen Glieder

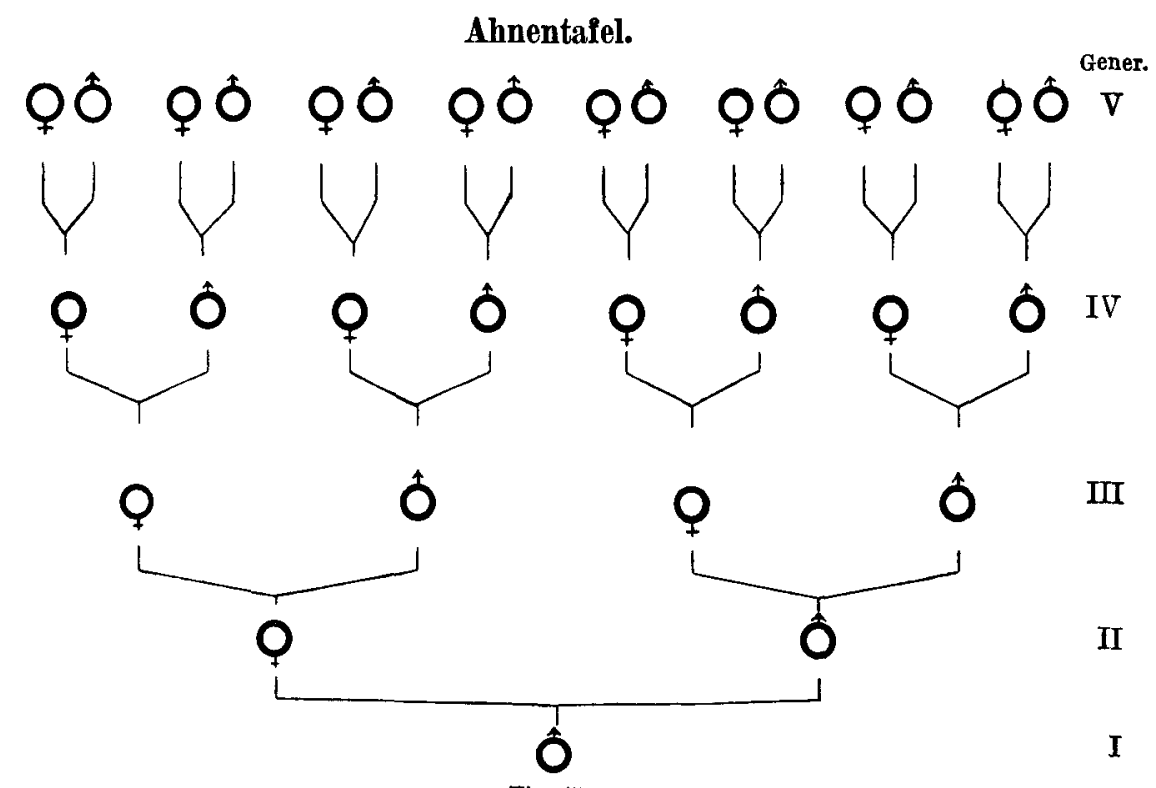

Fig. 15.

und über die wichtigsten und auffälligsten Befunde an den einzelnen Gliedern und Auskünfte über sie, sowie zur sofortigen Orientierung über die in der Materialsammlung noch bestehenden Lücken ein Schema des Familienaufbaues hergestellt, in welchem möglichst alle Verwandten eingezeichnet sind, sowohl die männlichen als die weiblichen Aszendenten, Kollateralen und Deszendenten. Wie ein solches UUbersichtssche ma oder Verwandtschafts-Schema etwa, in irgendeinem Stadium, aussieht, zeigt Fig. 14 (Tafel XXII).

Das Schema wird für die meisten Fälle, die zu unserer Beobachtung gelangen, für längere Zeit, während der die Materialsammlung fortdauert, genügen, denn es handelt sich für den Naturwissenschaftler, im Gegensatz zum historischen Genealogen, weniger darum, die Namen 
und äußerlichen Lebensschicksale möglichst vieler Ahnen oder möglichst vieler Deszendenten zusammenzubringen, sondern vielmehr darum, die Ahnen, Deszendenten und Kollateralen allseitig naturwissenschaftlich, d. h. nach ihren normalen und krankhaften körperlichen und seelischen Beschaffenheiten zu beschreiben, von denen wir wissen oder nur vermuten, daß sie sich vererben, $d . h$. im Verlaufe der Generationen sehr wenig abhängig von den äußeren Einflüssen sind, aber auch jene Einwirkungen auf ihr Leben sorgfältig zu registrieren, von denen wir wissen oder vermuten, daß sie die Betreffenden selbst oder ihre Nachkommen in dieser oder jener Weise verändern. Je gründ-

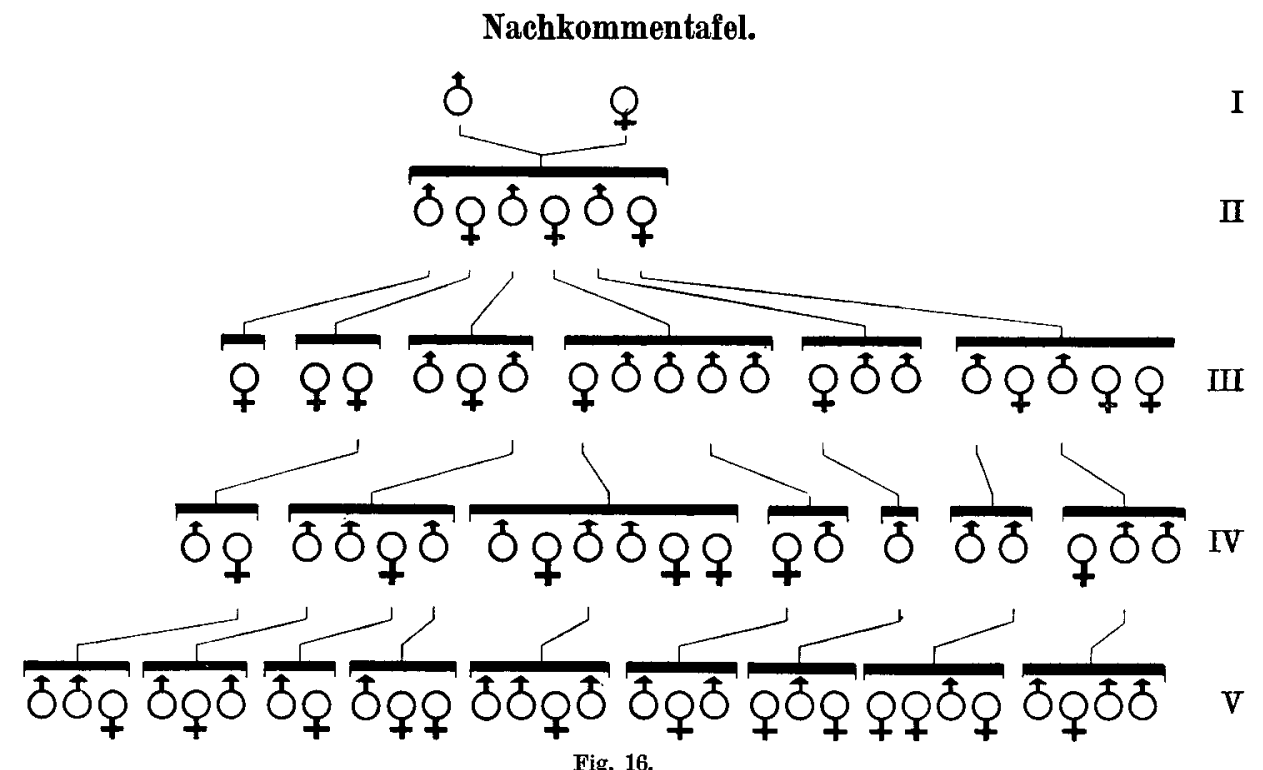

licher und intensiver die nächste wichtigste Verwandtschaft eines Probanden durchforscht wird, um so weniger rasch wird sich das Bedürfnis nach einer unhandlichen Ausdehnung des Utbersichts-Schemas ergeben, der jedoch leicht durch Anlegung eines neuen, mit dem alten leicht zusammenfügbaren Schemas ähnlicher Art vorgebeugt werden kann.

Je nach Einlaufen des Materials können auch andere Übersichten, wie die Ahnentafel (Fig. 15) oder die Nachkommentafel (Fig. 16) benutzt werden.

Doch sei ausdrücklich vor der einseitigen Verwendung der Ahnentafel oder der Nachkommentafel ge warnt, da jede Art der Darstellung wieder wertvolle Dienste leistet, die die andere nicht zu leisten vermag. 
So kann die Ahnentafel nur Aufschluß über gewisse Arten des Erbganges, über gewisse Arten von Belastung geben, nicht aber über die ebenso wichtige Va ria bilität, die voll und ganz nur in der Nachkommentafel zum Vorschein tritt. Doch ist die Nachkommentafel auch zur Demonstration des Erbganges für viele, nämlich die dominant nach Mendel gehenden Anomalien vortrefflich, ja allein geeignet, und es ist daher verhängnisvoll, wenn man in gewissen medizinisch-genealogischen Kreisen die Ahnentafel einseitig empfiehlt und kultiviert.

Eine Gegenüberstellung der Ahnentafel, z. B. mit Fig. 7, S. 498, wird auch sofort erkennen lassen, daß sie zur Erfassung recessiver Vererbung völlig unbrauchbar ist, ganz abgesehen davon, daß ihr die Darstellung einer der wichtigsten Seiten des Vererbungsvorganges, die Vererbungsproportion, gänzlich abgeht. Aber dasselbe gilt auch, für die recessiven Störungen, bei der Nachkommentafel, da die Anomalie ja durch angeheiratete Ahnen oder deren Geschwister hereinkommen kann, die auf der reinen Nachkommentafel gar nicht zu sehen sind. Diesem Utbelstand wird auch nicht ganz abgeholfen, wenn man auf der $\therefore$ achkommentafel jedem Familienglied den ehelichen Partner anhängt, da er ja selbst nur der Konduktor der Familienkrankheit sein kann.

Beide Tafeln sind gewiß gleich unentbehrlich, namentlich auch mit Rücksicht auf die Beziehungen der Vererbungsart und -Intensität zu Exogamie und Endogamie.

Immerhin wird zur Demonstration von Vererbungsvorgänge n die Ahnentafel wohl nur ausnahmsweise dienen können, vortreff lich in vielen Fällen (s. oben) dagegen die Nachkommentafel, a m besten eine Kombination von Ahnen- und Nach kommenschaftstafel nach der Art meiner Verwandtschaftstafel, die zumeist den wechselnden $S$ pezialbed ürfnissen der Demonstration angepa Bt werden kann und muß.

Denn man muß von vornherein darauf verzichten, alles, was Objekt der Forschung sein kann, auf ein Schema bringen zu wollen; denn jedes Problem, sei es die Vererbung von Krankheiten, von anthropologischen Eigenschaften, von Talenten, der Langlebigkeit, der Variabilität, der Fruchtbarkeit, Verteilung der Geschlechter usw. wird der figürlich-schematischen Darstellung in einer besonderen Utbersichtstafel bedürfen.

Dem einen oder anderen wird zur Übersicht über manche Verhältnisse auch Crzellitzers Sippschaftstafel (Fig. 17,) vortreffliche Dienste leisten. Doch muß auch hier vor ihrer einseitigen Verwendung gewarnt werden, unter anderm schon deshalb, weil in ihr die Utbersichtlichkeit über die Generationenzusammengehörigkeit erschwert ist.

Man kann also den Schluß, zu dem auch Sommer kommt, nicht genug beherzigen: 
Nur durch eine Vereinigung beider Hauptmethoden, nämlich durch Untersuchung der auf der Nachkommentafel dargestellten

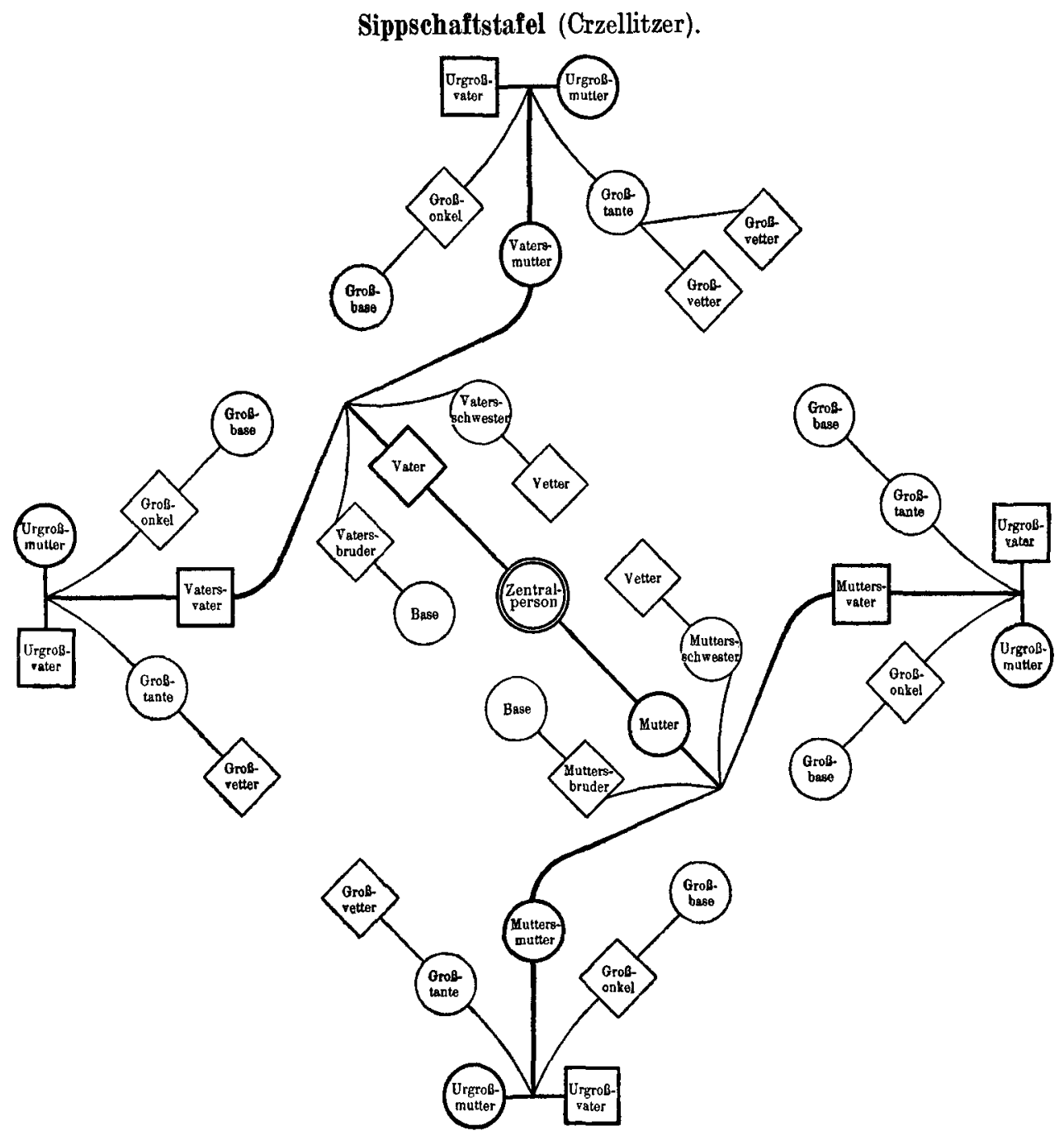

Zentralpersonen sind folgende Geschwister:
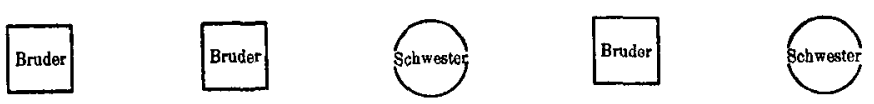

Fig. 17.

Blutsverwandten und derjenigen der Ahnentafel, lassen sich die Voraussetzungen zur Erkenntnis von Vererbungstatsachen - es muß aber 
beigefügt werden, auch Variationstatsachen - innerhalb der Familie schaffen.

Hand in Hand mit einer bei Einströmen eines großen Verwandtenmaterials stets wieder neu zu erzielenden Übersicht über die Blutsverwandtschaft und die auffälligsten Befunde in Gestalt von Verwandtschaftstafeln der obenerwähnten Art muß die für die Zukunft als wichtiger als alles andere zu bezeichnende ausführliche übersichtliche Registrierung sämtlicher eruierbarer Daten bezüglich aller Blutsverwandten gehen.

Das sollte mindestens in zwiefacher Weise geschehen.

Für den Problemenkreis, dessen Erforschung ich mir vorgenommen habe, benütze ich z. B. unter anderem die hier abgedruckte Fa milientafel (S. 534-539).

Jede Mutter mit ihrem Ehegatten und den gemeinsamen Kindern kommt auf eine solche Familientafel (Geburtenbogen), und aus diesen Tafeln stückt sich die ganze engere und entferntere Verwandtschaft des Probanden lückenlos zusammen. Ein Elternbogen (S. 537 -539) behandelt die Eltern noch getrennt, gewissermaßen in ihrer besonderen Eigenschaft als Zeuger. Die Tafeln können übersichtlich geordnet und zur wissenschaftlichen Verwertung leicht benützt und jede für sich verbessert und ergänzt werden. In sie sollen die Erhebungen, Beobachtungen und Untersuchungen vom Arzt selbst eingetragen werden, der sie gemacht hat. Sie können aber auch versandt und von Kollegen oder gebildeten Verwandten zum Teil ausgefüllt werden.

Auf diese Weise lassen sich die Daten in geordneter, stets leicht auffindbarer Weise und, wenn sie auf gutem Papier gedruckt sind, auf lange Zeit hin als Urmaterial aufbewahren.

Allein zahlreiche Verwandte bieten an charakteristischen Merkmalen aller Art bedeutend mehr, als auf dem beschränkten Raum der Familientafel Platz finden kann. Jedes Glied der Familie des Probanden, welches mehr bietet als auf der Familientafel Platz hat, erhält daher einen Personalbogen oder Personalakt, in welchem die Schulzeugnisse, die Lebensbeschreibung, literarische Produkte, ev. seine Krankengeschichten, seine Photographien, Auszüge aus Strafakten, Ehescheidungsakten, kurz alles, was die Persönlichkeit des Betreffenden kennzeichnet, verwahrt wird.

Die alphabethisch angeordneten Personalbogen werden mit den Familientafeln, Übersichtsschemata, Korrespondenzen (Kopien der Ausläufe) zur Beschaffung des Materials und Adressen von Auskunftserteilern, von Angehörigen usw. im Familienakt, welcher stets auf den Probanden lautet, zusammengeschlossen. 


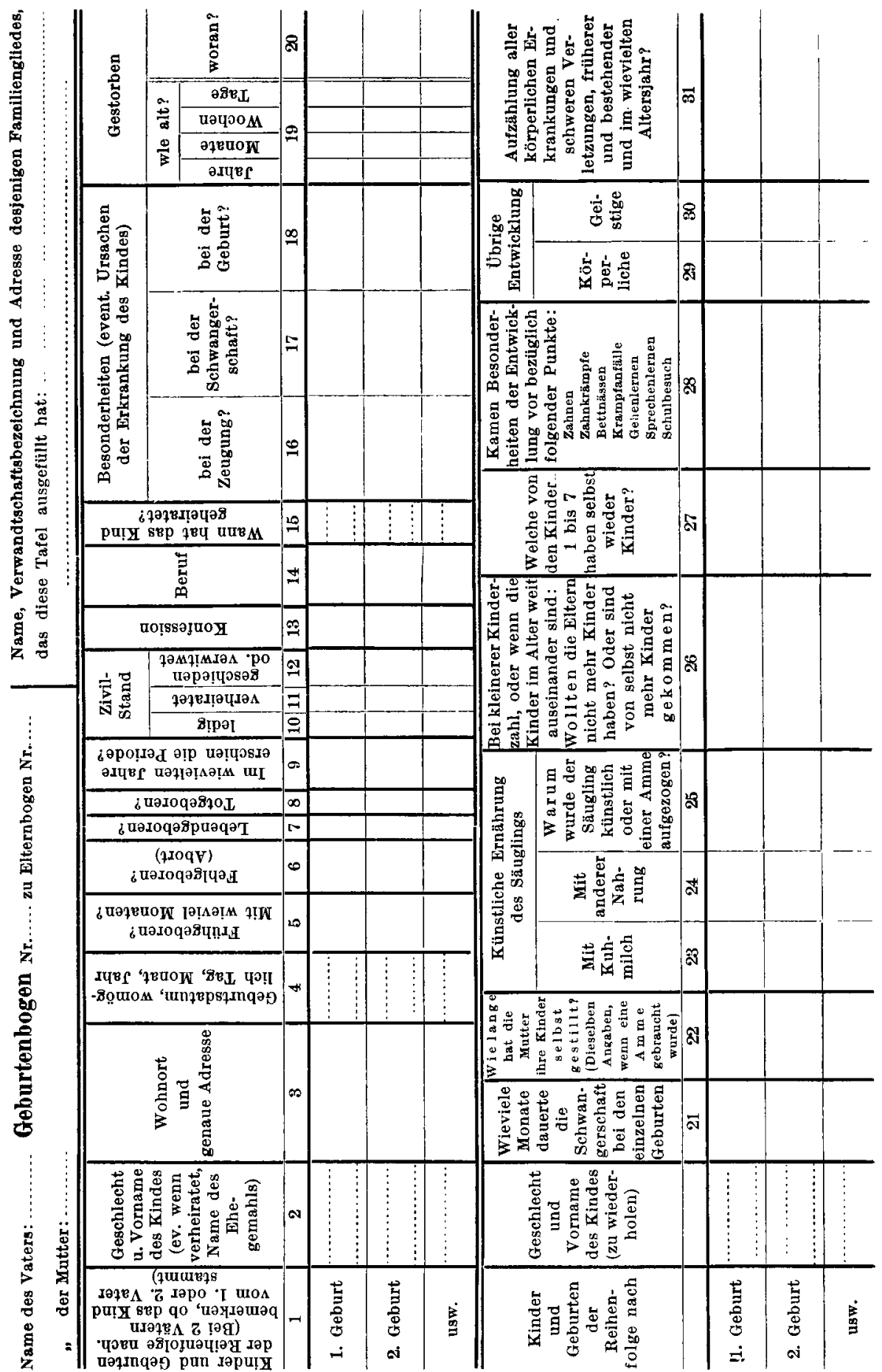


mit Rücksicht auf die Psychiatrie.

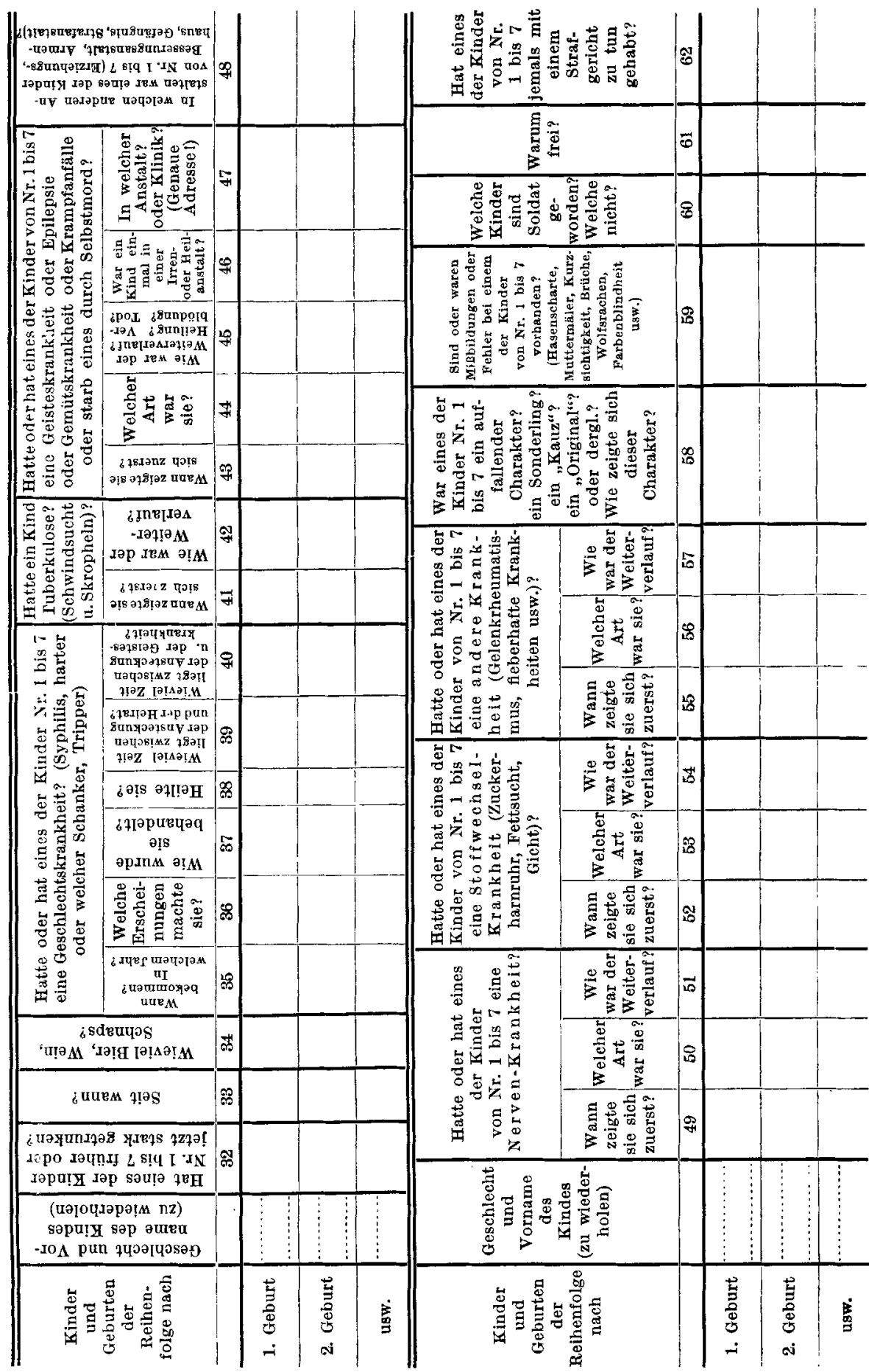




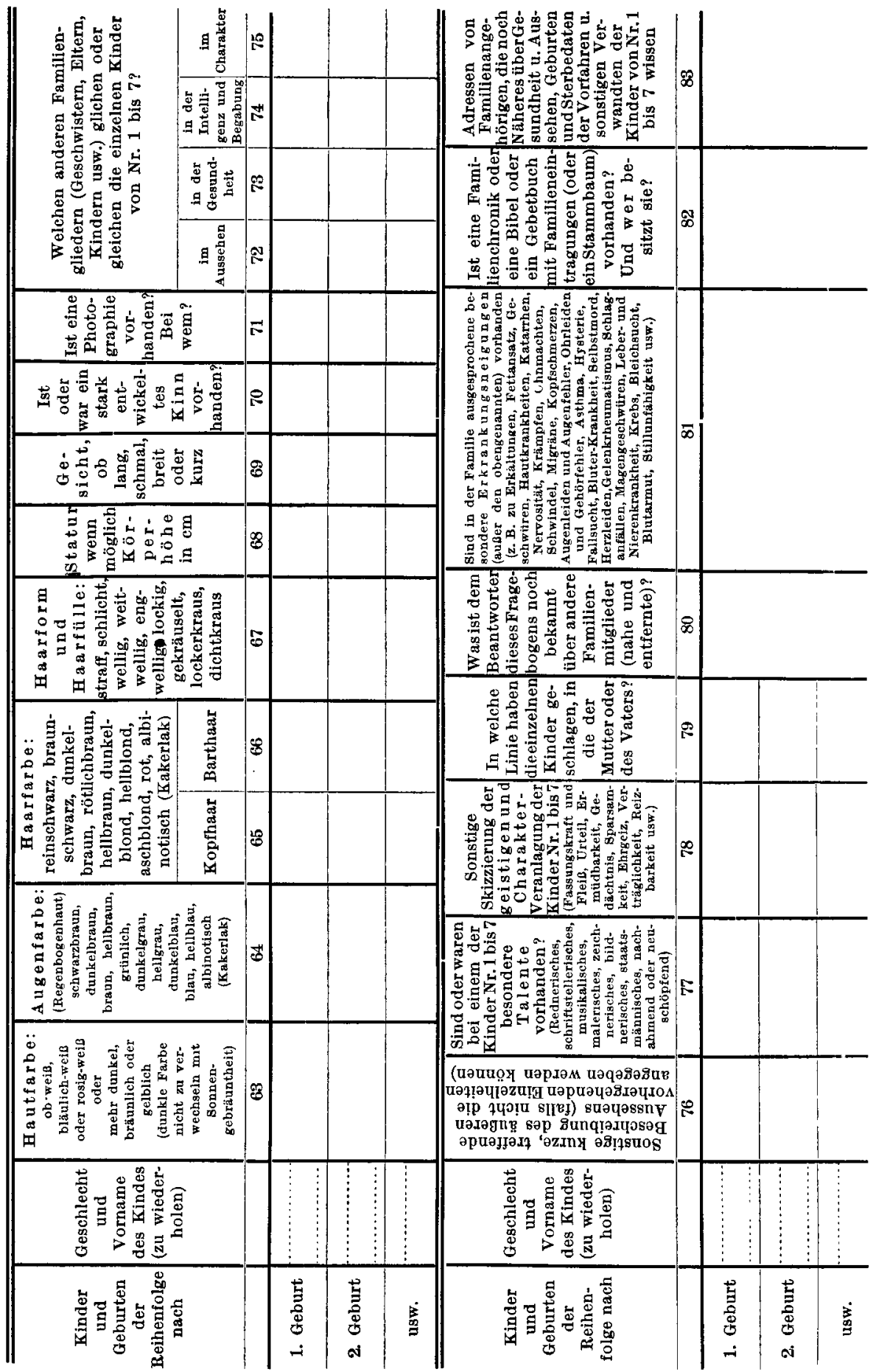


mit Rücksicht auf die Psychiatrie.

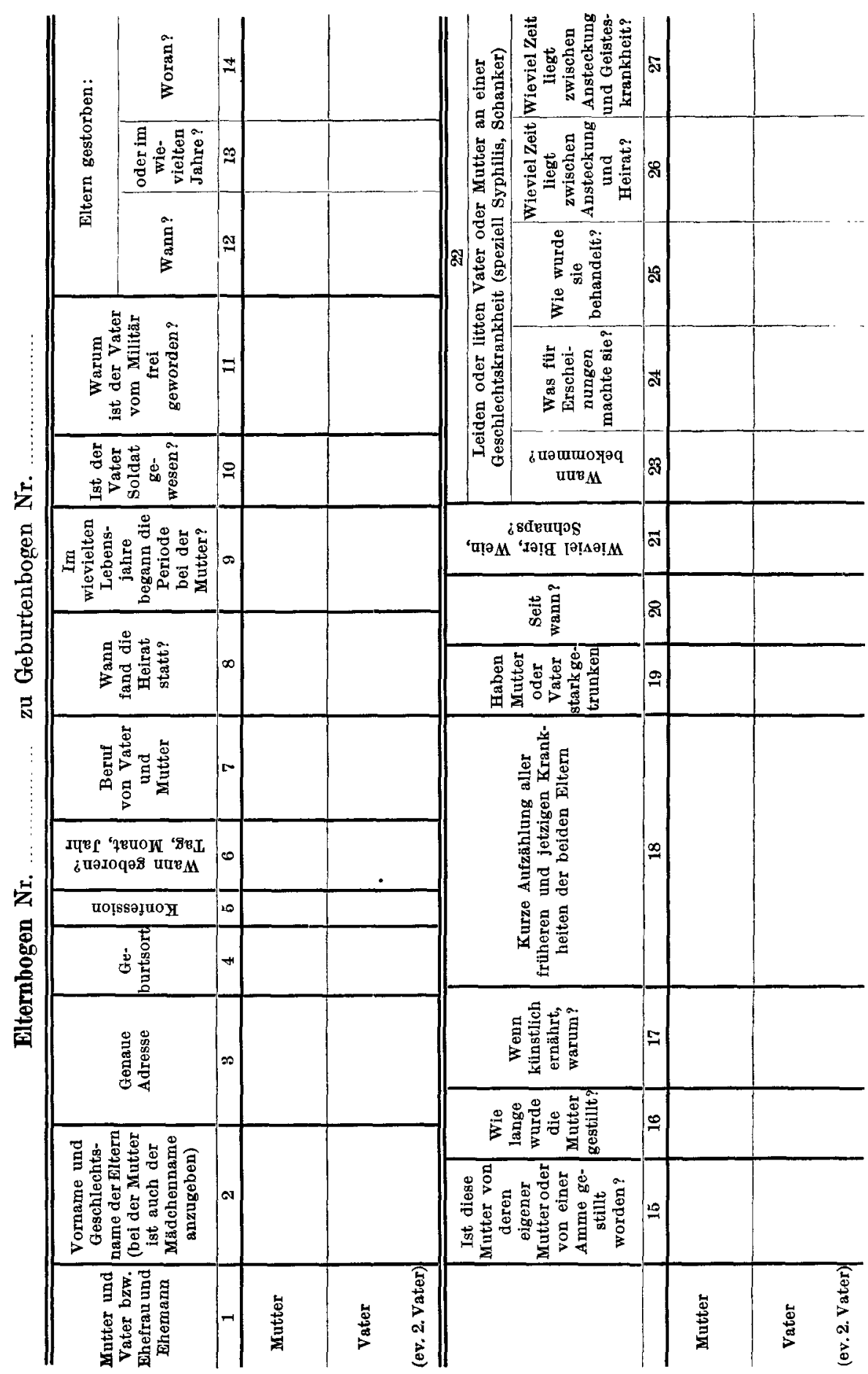




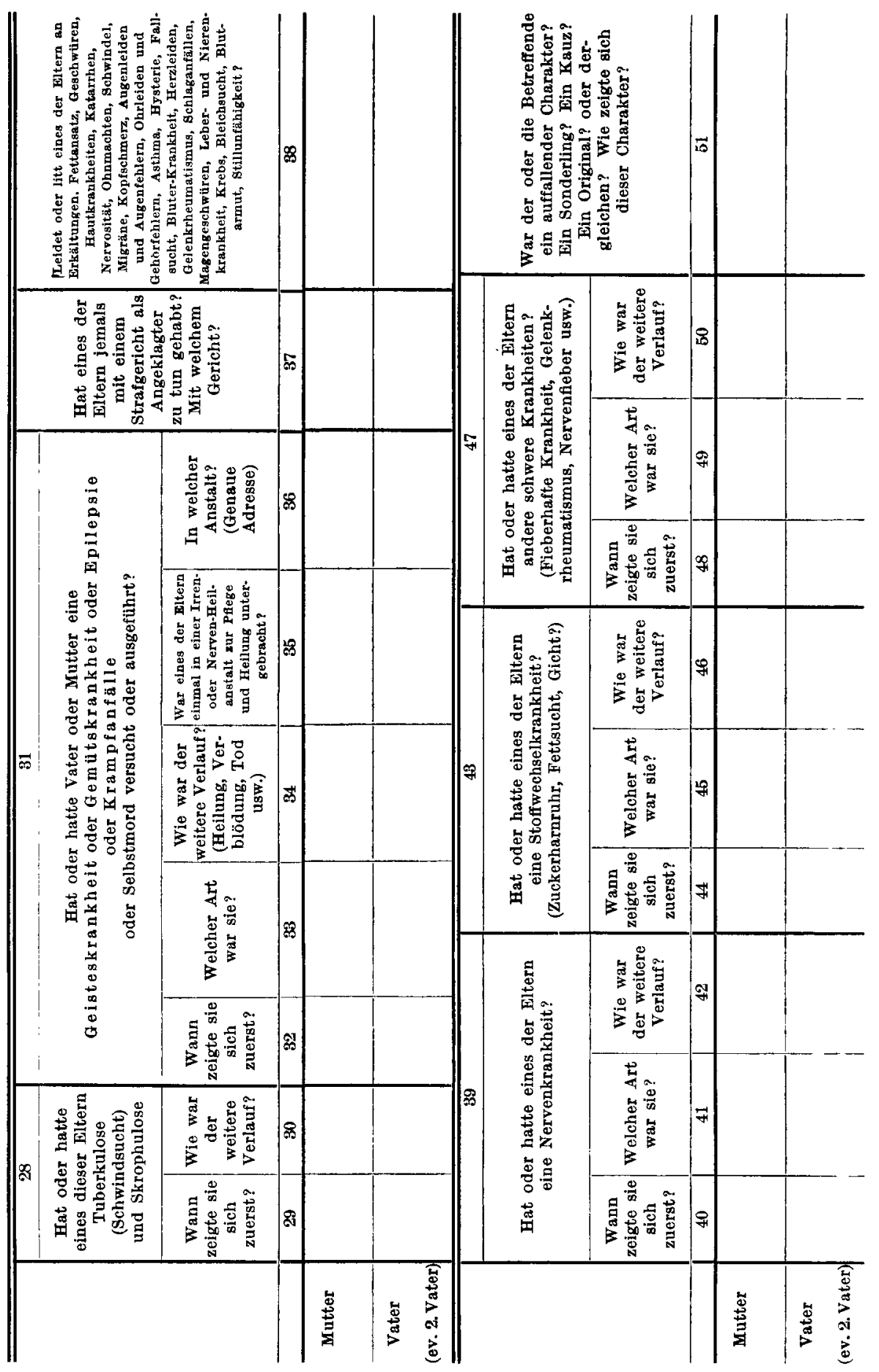


mit Rutcksicht auf die Psychiatrie.

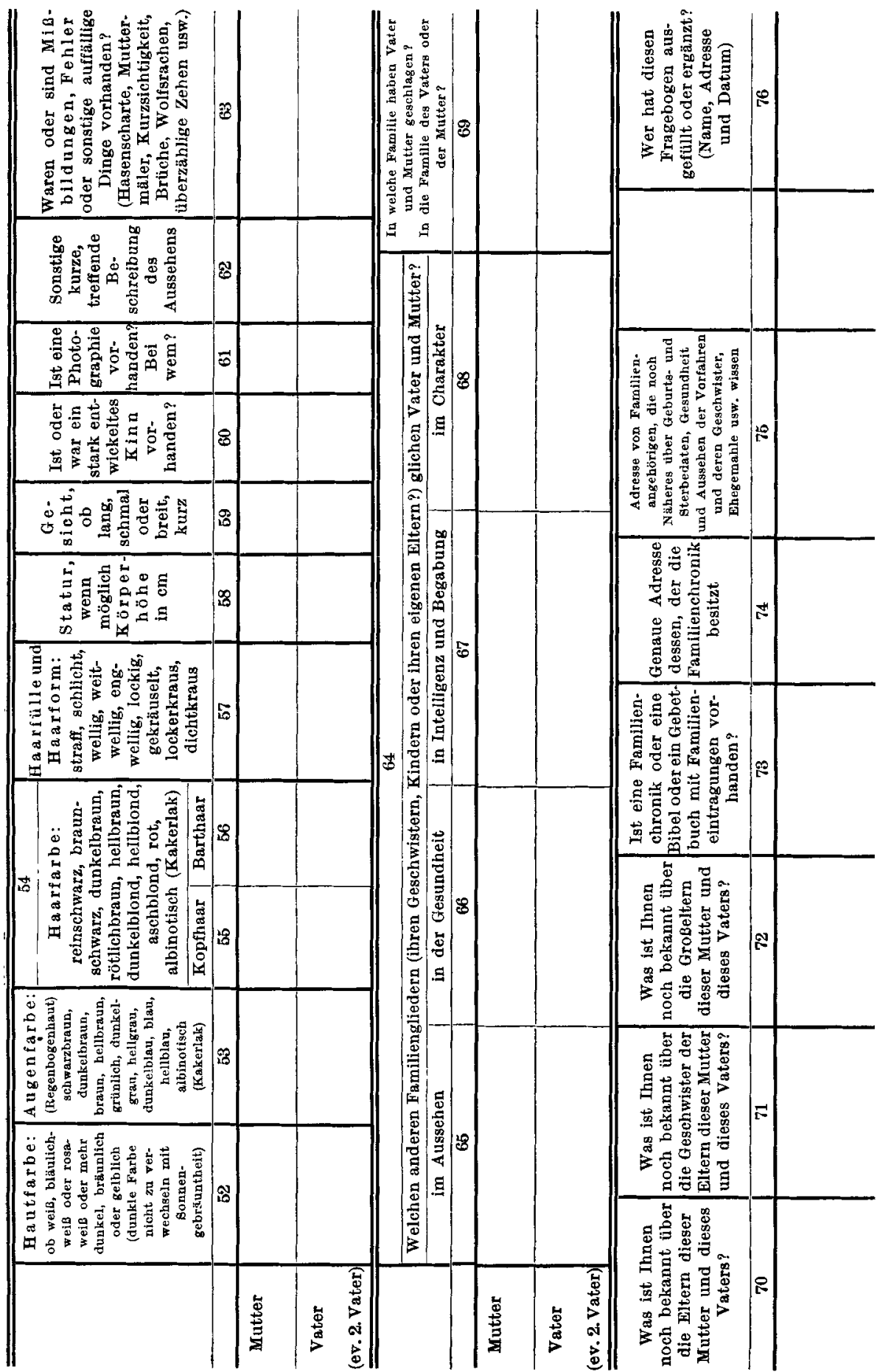


So kann neueinlaufender Stoff stets bequem dem alten beigefügt, der vorhandene sofort aufgefunden und wissenschaftlich verwertet werden. Zettelkataloge für die Probanden und, für die speziellen Bedürfnisse des Psychiaters, der kranken Verwandten der Probanden, gestatten die rasche Auffindung der Akten, Einordnung der Einläufe und Herstellung der gewünschten familienstatistischen Aufstellungen.

Als Mittel der Beschaffung des Materials, dessen Bezugsquelle stets vermerkt werden soll, kommen unter anderem in Betracht:

Die eigene Krankengeschichte des Probanden und eventuelle Krankengeschichten aus anderen Anstalten. Die persönliche, eingehende Befragung des Probanden über seine ganze Verwandtschaft, und zwar nicht bloß darüber, ob ,schon einmal etwas in der Familie vorgekommen", sondern über alles, was ihm von den einzelnen Gliedern bekannt ist und was als Material zur Problemlösung in Betracht fällt. Wünschenswert ist eventuell die photographische Aufnahme und die anthropologische Registrierung des Probanden, wichtig die Bestellung und persönliche Befragung möglichst aller erreichbaren Verwandten des Probanden, die Aufzeichnung mündlicher Familientraditionen.

Ferner: die eigenen und fremden Krankengeschichten von erkrankten Verwandten des Probanden (Abschriften), die Auskünfte von Pfarrämtern (Kirchenbücher) und Standesämtern, der Polizei, der Heimatgemeinden, der Armenverbände, der Gerichte (Strafakten, Ehescheidungs-, Entmündigungsakten), der Schulen, der Erziehungsanstalten, Anstalten für Schwachsinnige, Trinkerasyle, Krankenhäuser, Militärbehörden usw., über den Probanden und dessen Verwandte. Dazu können, den lokalen Verhältnissen entsprechend, geeignet abgefaßte Formulare benutzt werden.

Natürlich sollen vom medizinischen Genealogen a uch alle Quellen benutzt werden, aus denen der historische Genealoge zu schöpfen pflegt: Biographien, Standeslisten, Adreßbücher und Personalverzeichnisse aller Art, welche die Information erleichtern, Familienchroniken usw., Urkunden aller Art, Geburtsscheine, Taufscheine, Grund- und Lagerbücher, alte Verträge, Schuldscheine, Ausgabenverzeichnisse, Personalakten aller Art (Beamte), frühere „Dienerbücher", Grabsteine, Todesanzeigen usw. in den Zeitungen, Leistungen der Familienglieder aller Art: schriftstellerische, malerische usw.

Außerordentlich wichtig für den Psychiater ist auch der Besitz der Personalien geisteskranker Internierter oder interniert Gewesener für den engeren oder weiteren Bezirk, innerhalb welchem er seine Untersuchungen vornimmt, da dadurch die Feststellung blutsverwandtschaftlicher Beziehungen außerordentlich gefördert wird. Hier würde ein Zusammengehen und eine gegenseitige Unterstützung aller Psychiater 
eines Landes, z. B. Bayerns, eine gewaltige Erleichterung für den Familienforscher bedeuten.

Was die genealogische Bezifferungsmethode anbetrifft, so haben ja Lorenz und Sommer in dieser Richtung vortreffliche Vorschläge gemacht. Im ganzen ist hier aber, wie in der übrigen genealogischen Terminologie, noch alles ziemlich der Willkür des einzelnen anheimgegeben, und man hat daher eine internationale Regelung dieser Dinge vorgeschlagen. Das ist auch sehr zu begrüßen, wenn auch darauf Bedacht genommen werden muß, die Vielfältigkeiten, namentlich in der figürlichen Darstellung, welche in der Vielfältigkeit der Naturvorgänge begründet sind, nicht in das Prokrustesbett einer rein historisch-heraldischen Genealogie zu zwängen. Der medizinische Genealoge hat allen Anlaß, in dieser Hinsicht sich nicht nach der historischen Genealogie, sondern nach der experimentellen Biologie zu richten.

Wie ich bereits bemerkte, muß es unser Bestreben sein, zur Aufklärung der Vererbung dieselben ausgedehnten und lehrreichen Zuchten durch Materialanhäufung und Materialauswahl zusammenzubringen, welche die Pflanzen- und Tierzüchter experimentell, durch planmäßige, rationelle Züchtung der verschiedenen Bastardgenerationen erhalten haben, um dann aus den sorgfältig und nach wissenschaftlichen Methoden charakterisierten Einzelgliedern Proportionengruppen von gleichartigen Individuen zu bilden. Es ist sicher, daß wir dann für manche Merkmale dieselben, an die theoretisch erwarteten außerordentlich angenäherten, wirklichen Proportionenwerte erhalten werden, wie die Botaniker und Zoologen.

Inzucht der ersten Bastardgeneration fällt praktisch beim Menschen nicht erheblich ins Gewicht, wenn auch in manchen Gegenden Blutschande nicht so selten ist. Allein ein gewisser, wenn auch mühsam zu beschaffender Ersatz liegt doch in der genauen Durchforschung der Vorfahren der Variante, deren Erbwert zu bestimmen ist, vom Zoologen aber verhältnismäßig mühelos durch wahlvolle Kreuzungskombinationen bestimmt wird.

Es sollte daher unsere Hauptaufgabe sein, wenigstens für die $\mathrm{Zu}$ kunft die Registration möglichst zahlreicher und vollständiger Generationen zu beschaffen.

Unterdessen aber wird die statistische Verwendung schon einer geringen Anzahl von Generationen zu versuchen sein.

Wie dies zu geschehen hat, haben Davenport, Bateson und Weinberg zu zeigen versucht.

Einige Hinweise, wie man zu überlegen hat, ob gegebenenfalls Mendelsche Vererbung vorliegt, werden nützlich sein. Zunächst sei in diesem Zusammenhang nochmals auf die Eigenart der dominanten Merkmale, wie sie z. B. in Fig. 3, S. 492 klassisch zutage tritt, hingewiesen. 
Die Eigentümlichkeiten lassen sich im allgemeinen daran erkennen, daß sie der Regel der sog. direkten kontinuierlichen Vererbung folgen und nur durch die von den dominanten Eigenschaften affizierten, also das dominante Merkmal äußerlich zur Schau tragenden Glieder der Familie vererbt werden, wogegen für die nichtaffizierten die Regel „Einmal frei, immer frei“ gilt, das pathologische Merkmal also bei den Nachkommen eines normalen Elternpaares definitiv verschwindet, wenn es sich nicht bei einem solchen Nachkommen aus anderen Ursachen, die mit Vererbung nichts zu tun haben und später besprochen werden sollen, findet.

Bezüglich der Proportionen, welche für das Vorliegen Mendelscher Dominanz einer Affektion sprechen, verweise ich auf Fig. 9, S. 501 .

Wo Affizierte unter sich heiraten, sind die Proportionen der Rubriken 2, 4 und 5 zu erwarten, und zwar bei intensivster Inzucht (Endogamie) sowohl, als auch bei weitgehendster Fremdkreuzung (Exogamie).

Wo im affizierten Stamme die affizierten Individuen nur Gesunde heiraten, werden die Proportionen der Rubrik 3 gefordert, die Hälfte der Nachkommen krank. Nur wenn einmal im Stamme auch eine Heirat zwischen Affizierten vorgekommen ist (Rubrik 5), ist für die Nachkommen die pathologische Vererbungskraft größer, nämlich nach den Proportionen der Rubrik l (kommen dagegen mehrfach Heiraten zwischen Affizierten vor, so vermehren sich auch die Proportionen der Rubriken 1, 2 und 4) mit den respektiven Folgen für die weitere Nachkommenschaft.

Ist in einer größeren Geschwisterzahl ein bestimmtes, gut definiertes, nicht etwa durch Diagnosenkunststücke identifiziertes Merkmal bei der Hälfte der Individuen vorhanden, so wird man sofort an ein dominant mendelndes Merkmal denken und nach seinen anderen Kriterien und Auswirkungen in der Familie fahnden.

Allein in dieser Richtung sei man vorsichtig. Wie ein Blick auf Fig. 8, S. 499, unterste Generation, lehrt, können diese Proportionen bestehen, aber es kann doch recessive Abnormität vorliegen. Eine eventuelle erste Ehe des nichtaffizierten Elters mit einem ebenfalls gesunden Partner, aus der mindestens ein krankes Kind (Fig. 8, S. 499, jüngste Generation, 1. Familie) hervorgeht, kann dann volle Klarheit bringen über die recessive Natur der vorliegenden Störung.

Ist der Verdacht vorhanden, daß eine geschlechtsgebundene Dominanz einer Abnormität vorliegt, so müssen dem Forscher die Verhältnisse der Fig. 11, S. 508 oder ihre UUbertragung auf das weibliche Geschlecht vorschweben.

Wo die Affektion den Verdacht erweckt, dominant bei männlich 
$\mathrm{zu}$ sein, tritt sie im allgemeinen (siehe jedoch für Genaueres das Schema der Fig. 11, S. 508) nur bei den männlichen Individuen auf. Durch nichtaffizierte Männer wird sie nicht übertragen, durch die affizierten Männer aber je nach ihrer und ihrer Frauen Zusammensetzung auf alle oder nur auf einen Teil der Söhne. Sie kann aber auch durch nichtaffizierte Frauen vom Großvater auf den Enkel übertragen werden. Wo der Zustand der Affektion dies verträgt (was bei Bluterfrauen natürlich nicht der Fall ist) kann, wie bei der Farbenblindheit, die Anomalie auch bei den Frauen auftreten, wenn sie nämlich, wie Fig. 11 zeigt, von zwei Heterozygoten abstammen und selbst Homozygote sind. Dann sind alle ihre Söhne affiziert.

Der Verdacht, daß die Vererbung eines recessiv gehenden Merkmals vorliegt, ist dann begründet, wenn zwar ebenfalls mehrere Glieder der Familie befallen sind, aber in der Weise besonders, daß die Affizierten Kinder gesunder Eltern sind (Fig. 10, Rubrik 5, S. 502) ein Viertel der gesamten Geschwister ausmachen und selbst wieder gesunde Kinder haben (Fig. 10, Rubrik 1). Allein in schwerer belasteten Familien sind auch die Proportionen der Rubriken 2 u. 4 zu erwarten, also direkte Erblichkeit, und es wäre eine verhängnisvolle Unterlassung, diese Möglichkeit nicht stets vor Augen zu halten. Verwandtenheirat, also Inzucht, ist dem Auftreten und der Erhaltung recessiver Individuen günstig, exogame Verbindungen von Gliedern aus Familien mit recessiven Erbübeln mit gesunden Gliedern eines gesunden Stammes können das recessive Leiden praktisch ganz zum Verschwinden bringen.

Die recessive Eigenschaft kann mehrere Generationen hindurch völlig latent vorhanden sein, um plötzlich, wenn zwei Heterozygote (Fig. 10, Rubrik 5) zusammentreten, manifest zu werden.

Hat ein recessiv kranker Elter kranke Nachkommen, so muß sein Partner entweder selbst in derselben Weise krank sein (Rubrik 4) oder recessive Anlage von irgendwelchen, wenn auch noch so entfernten Verwandten mit sich führen, wenn das seiner äußeren Gesundheit auch nicht anzusehen ist (Fig. 10, Rubrik 2).

Sind beide Eltern recessiv affiziert, so sind alle Kinder affiziert (Fig. 10, Rubrik 4).

Ist nur ein Elter recessiv affiziert, so sind, bei Belastung des anderen Elters mit der gleichnamigen Anomalie in der Regel (nämlich wenn ein heterozygoter Partner vorliegt, Rubrik 2) die Hälfte der Kinder krank. Nur wenn der Partner aus der belasteten Familie ein Homozygoter ist, was bei entfernter Belastung oft vorkommt, sind auch alle Kinder gesund. Dieser Fall tritt auch ein, wenn der Partner nicht belastet ist (Rubrik 1). Verbinden sich zwei gesunde Homozygote aus einer belasteten Familie, so sind ibre Kinder ebenfalls gesund, sie bilden für die Nachkommenschaft keine Gefahr mehr (Rubrik 6). 
Allein auch alle oder zum Teil (Rubrik 3) heterozygoten Kinder aus recessiv belasteten Familien sehen äußerlich (nach der Theorie) gleich aus, geben aber bei Verbindung untereinander doch wieder Affizierte, so daß eine recessive Anomalie in praxi nur durch dauernde Rückkreuzung auf einen anomaliefreien Stamm, also dauernde exogame Heirat mit Gesunden, aus dem kranken Stamm herauszubringen ist.

Nur dem recessiven Vererbungsmodus können schließlich jene Anomalien folgen, deren Träger, wie bei der amaurotischen Idiotie oder vielleicht auch bei der Pelizä us-Merzbacherschen Krankheit, überhaupt nicht ins zeugungsfähige Alter kommen. Wie diese Anomalien erstmals entstehen, ist rätselhaft. Wir müssen wohl annehmen durch primäre Keimvariation. Allein nachdem sie einmal entstanden sind, bietet der recessive Mendelismus wohl die einzige Erklärungsmöglichkeit ihrer „UUbertragung", der ja fordert, daß, wo in einer Generation recessive, also kranke Homozygote, zu finden sind, stets auch die entsprechenden Proportionen von gesund aussehenden, aber die kranke Anlage beherbergenden Heterozygoten vorhanden sein müssen, wodurch dann bei weiteren Kreuzungen (erfahrungsgemäß besonders in Verwandtenheiraten) mit gleichanlagigen, äußerlich gesunden Heterozygoten immer wieder rezessive, affizierte und also nicht zeugungsfähige Homozygote entstehen müssen.

Beim Menschen, dessen Fortpflanzungsverhältnisse nicht durch die mächtige Hand eines Spezialzwecke verfolgenden Züchters gelenkt werden, treten im äußerlich sichtbaren Resultat eines gegebenen Vererbungsmodus zahlreiche Störungen ein, welche man als Forscher ebenfalls stets vor Augen haben muß, weil dadurch unter anderem Proportionen entstehen können, welche mit den nach Mendel zu fordernden nicht in Einklang zu bringen sind.

Ganz abgesehen von rein äußerlichen Beobachtungs- und Überleferungsschwierigkeiten, gegen die man mit großer Langmut gerüstet sein muß, greifen die a uslesende $Z$ uchtwahl und die Ausmerze mächtig in die Mendelschen Zahlenverhältnisse ein, weshalb man bei Nichterfüllung der erwarteten Proportionen den allgemeinen biologischen und sonstigen pathologischen Vorgängen in den Familien des ganzen Stammes stets eine besondere Aufmerksamkeit schenken muß.

So kann die verschiedene Fruchtbarkeit in den Familien, welche einen kranken Stamm zusammensetzen, erhebliche Verschiebungen bewirken. Denn soweit bis jetzt bekannt, folgt z. B. die Geburt einer kranken und gesunden Variante einer dominant gehenden Anomalie durchaus nicht etwa so, daß die Geburt eines Gesunden mit der eines Abnormen regelmäßig abwechselt, sondern die Abnormen können z. B. zuerst gehäuft vorkommen oder aber umgekehrt auch vorwiegend als Letzt- 
geborene. Ist nun aber die Fruchtbarkeit im Stamm eine geringe und will der Zufall, der nur bei großem Material vermieden werden kann, daß die Abnormen in mehreren Familien des Stammes vorwiegend vornedran in der Geburtenreihe sind oder umgekehrt die Normalen, so können die erhaltenen Zahlenwerte mit den Mendelschen Proportionen nicht übereinstimmen; sie sind für die Abnormen zu groß im ersten, zu klein im letzteren Falle. Ja für eine Einzelfamilie mit wenig Kindern wird sogar oft genug der Fall eintreten, wo z. B. unter 3 oder 4 Kindern, besonders häufig unter zweien, der Mendelschen Regel ganz zuwider, überhaupt keine Abnormen oder lauter Abnorme vorhanden sind.

In erhöhtem Maße hat man sich dieser Art Störung bei den recessiv gehenden Anomalien zu versehen.

Ein Blick auf Fig. 8, S. 499 (jüngste Generation) zeigt sofort, wie sehr eine geringe Fruchtbarkeit, z. B. das Zweikindersystem, völlig falsehe Proportionen vortäuschen kann. Auch hier muß man sich daher stets alle Erbmöglichkeiten bei größerer Fruchtbarkeit, sowie die Auswirkungen des vermuteten Vererbungsmodus in den Familien der Aszendenz und der Seitenlinien vor Augen halten.

Man beherzige daher ganz allgemein den Grundsatz, das gesamte Stammaterial so anwachsen zu lassen, daß den zwiefachen Elementen, welche die Proportionen erfüllen sollen, der gehörige Generationenspielraum zur Entfaltung gegeben wird. Er muß um so größer sein, je kleiner und je unregelmäßiger die Fruchtbarkeit der einzelnen Familien des kranken Stammes ist.

Vielleicht wird später ein größeres Material umgekehrt uns dann einmal auch Rückschlüsse zu ziehen gestatten auf eine gewisse Regelmäßigkeit in der Reihenfolge der dominant- und recessivmerkmaligen Geburten und uns vielleicht davon überzeugen, daß sie doch nicht einem so großen Würfelspiel unterworfen ist, wie dies von vornherein hier ja wohl zu begreifen wäre.

Ein weiteres störendes Element der Proportionserfüllung ist die Sterblichkeit.

Selbst wo die Anomalien bei der Geburt schon sichtbar sind, stört die Sterblichkeit vielfach aus rein äußeren Gründen, weil die Anomalie bei Totgeborenen und Frühgestorbenen oft genug gar nicht konstatiert wird oder, namentlich wo frühere Generationen in Frage kommen, vergessen wird.

Auch ist die außerordentlich wichtige Möglichkeit zu beachten, daß eine Variante, z. B. die kranke dominante, eine höhere Sterblichkeit besitzen kann, als die recessive oder umgekehrt.

So weiß man z. B., daß die Nesseln mit geradem Blattrande (Urtica pilulifera), welche sich bei Kreuzungen mit Nesseln mit gesägtem 
Blattrande (Urtica Dotartii) einfach recessiv verhalten (analog Fig. 1, S. 488, Erbsen) gegen Pilzkrankheiten merklich empfindlicher sind, als die gesägtrandigen Pflanzen, so daß das Verhältnis immer mehr zugunsten der letzteren verschoben wird, je später man die Durchzählung der Nachkommen in der zweiten oder dritten Bastardgeneration vornimmt. Das klassische Verhalten $3: 1$ ist hier also, aus dem genannten äußeren Grunde, fast nie genau vorhanden.

Durch sofortiges Fassen der Proportionen bei ihrer Entstehung kann diesem Umstande abgeholfen werden.

Doch muß damit gerechnet werden, daß die Träger gewisser Anlagekombinationen überhaupt nicht lebensfähig sind.

Am störendsten wird die Sterblichkeit in den Familien, wo, wie bei den meisten Geistesstörungen, Anomalien erblich sind, welche erst später im Leben hervortreten, wie bei gewissen Anlagekrankheiten, und also, selbst bei den besten Beobachtungsmöglichkeiten, vor Eintritt eines gewissen Alters überhaupt nicht konstatiert werden können.

Je später also eine Anlagekrankheit bei den einzelnen behafteten Gliedern des Stammes auftritt, um so größer werden natürlich die Chancen, daß die die Anlage zur Entfaltung der Krankheit in sich bergenden Träger aus diesem oder jenem Grunde der Ausmerze verfallen, bevor sie, dem Gesetze des Krankheitsbeginns in bestimmtem Alter folgend, Zeit hatten, krank zu werden. Diese Fälle müssen stets als ein definitiver Verlust für die zu fordernden Affiziertenproportionen darstellen, welcher auch nicht leicht durch eine Häufung des Materials gut zu machen ist.

In solchen Fällen wird zu untersuchen sein, ob die fehlenden Proportionen der Affizierten in dem Maße sich einstellen, als der Prozentsatz der Glieder der zum Vergleich herbeigezogenen Familie steigt, welche das Alter zumindest der untersten Grenze des Krankheitsbeginns, besonders aber derjenigen, welche das Alter der obersten Grenze des Krankheitsbeginns erreicht haben.

Sind die geschilderten Mängel unabstellbar und ist nichts vorhanden, was die Annahme der Mendelschen Vererbung direkt unmöglich macht, so wird man, wenn man dennoch Schlüsse ziehen will, sich vorsichtig ausdrücken und etwa sagen, daß zwar die vorliegenden Tatsachen nicht genügen, um das Vorliegen Mendelscher Vererbung positiv zu beweisen, daß aber auch doch nichts positiv dagegen spricht.

Die geschilderten Mängel treten schon bei Krankheiten wie der Dementia praecox deutlich hervor, am störendsten natürlich bei Arteriosklerose und seniler Demenz, deren Erbgang eigentlich einwandfrei nur studiert zu werden vermag in Familien mit allgemeiner, weitverbreiteter Langlebigkeit all ihrer Glieder. 
Man bedenke auch die wichtige Tatsache, daß in ein und demselben Stamm eine Anomalie dominant (oder recessiv) bei den männlichen Gliedern, eine andere, vielleicht sogar ähnliche Anomalie dominant (oder recessiv) nur beim weiblichen Geschlecht sein kann.

Ein wichtiges Moment der Störung kann auch die mangelhafte Periodizität in der Geschlechtsfolge der Geborenen abgeben.

Zweifellos ist die korrelative Verknüpfung des Geschlechtes mit abnormen Eigenschaften keine Seltenheit. Ist die Sexualperiodizität, die Aufeinanderfolge von männlichen und weiblichen Geburten eine regelmäßige, so kann dieser Umstand bei Affinität eines bestimmten Geschlechts für eine bestimmte Erkrankung zur großen Regelmäßigkeit und leichten Erkennbarkeit des Erbbildes und der Erbproportion führen. Andererseits aber kann im selben Falle eine unregelmäßige Geschlechtsfolge oder gar eine einseitige Geschlechtsproduktion im höchsten Maße verwirrend auf die Proportionenseite des Erbbildes wirken. Auch diesem Punkt muß daher stets die schärfste Aufmerksamkeit zugewendet werden.

Ein nicht zu unterschätzendes störendes Moment kann darin begründet sein, daß Erbanlagen erst zum Vorschein kommen unter ganz besonderen physiologischen Vorbedingungen oder Vorbedingungen des Milie us, womit wir in der Psychiatrie zweifellos besonders häufig zu rechnen haben. Eine Hauptklippe geistiger Gesundheit ist für manche Psychosen und Individuen das Wochenbett. Fehlt dieses aber, infolge Ledigbleibens, bei einer größeren Anzahl weiblicher Familienglieder, so können die Erkrankungsproportionen dadurch erheblich alteriert werden.

Dasselbe gilt natürlich für alle Psychosen und Psychopathien, die, wenn schon zum Teil zweifellos auf Anlage beruhend, andererseits doch auch unbedingt der Auslösung durch bestimmte äußere Reize oder Reize anderer Körperteile bedürfen, um auszubrechen.

Ich erinnere nur an die alkoholischen Psychosen, an die Paralyse, an die traumatischen Hysterien u. dgl.

Die Proportionenfrage gestaltet sich hier zu einer ganz außerordentlich schwierigen Aufgabe, und man wird sich daher in diesem Falle mit ihrer Anwendung auf die respektiven präpsychotischen Persönlichkeiten begnügen müssen.

Jedenfalls geht aus diesen Erwägungen über die Auslösungspsychosen hervor, von welch ungeheurer Bedeutung für den Erbbiologen die genaue Diagnosenstellung ist, sowie daß Methoden in Anwendung kommen, welche in exakter Weise die Eigenart der präpsychotischen Gesamtpersönlichkeit festzustellen vermögen, damit ihre sichere Zuordnung zur später psychotischen Persönlichkeit unter den respektiven Außenbedingungen einst vorgenommen werden kann. 
Das Vererbungsproblem an und für sich ist, wie wir gesehen, gewiß ein recht wichtiges Forschungsgebiet des Psychiaters, auf welchem ungeahnt viele, theoretisch wie praktisch gleichwichtige exakte Ergebnisse zu ernten sind. Der Weg ist vom Experimentator vorgezeichnet. Für uns führt er durch die ernste, berufsmäßige Familienforschung. Wir brauchen ihn nur zu gehen.

Allein ebenso bedeutsam, ja vielleicht noch wichtiger ist das Studium der Variabilität, d. h. der Veränderlichkeit der Merkmale und Merkmalskomplexe des Menschen.

Das Studium des Veränderungsvorganges, welcher zu Neuerscheinungen führt, und seiner Ursachen ist demjenigen der Vererbung mindestens ebenbürtig, da es uns die lebenswichtigste aller Fragen, welche die höherstrebende und leidende Menschheit an sich richten kann, zu lösen verspricht, wie einerseits außergewöhnliche Begabung und Tüchtigkeit, anderseits Defektheit, Krankheitsanlage und Krankheit erstmals entstehen, erstmals in einer Familie, einem Stamm auftauchen, um dann erst nach den Regeln der Vererbung übertragen zu werden.

Vererbung ist starre Überlieferung: Wo bliebe in der Welt die Entwicklung nach oben, der Fortschritt, wo bliebe die Entwicklung nach unten, die Entartung, wenn es nur die Vererbung gäbe. Vererbung ist Konservativität. Wo solche also nicht vorhanden ist, muß der erste Gedanke sein, nach den Ursachen zu suchen, welche sie gestört haben. Das sollte das Leitmotiv aller Ursachenforschung sein. Folgt man blind nur dem einen Leitmotiv der Erblichkeit, so zwingt man naturgemäß künstlich Phänomene in sie hinein, welche ganz offenkundig nicht in sie hinein gehören, und so wird dann schließlich alle Variabilität zu Vererbung, und somit zu einem begrifflichen Zerrbild, das jeglicher gesetzmäßiger Grundlage und jeglichen Wertes entbehrt, weil es Vorgänge umfaßt, die entgegengesetzte Wirkungen entfalten und verschiedene Ursachen haben.

Ich fasse dabei den Begriff der Vererbung ganz außerordentlich weit, indem ich daran erinnere, daß auch der Kreuzungsrückschlag ganz zweifellos ein Phänomen Me ndel scher Recessivvererbung sein kann, bei der, nicht anders als bei den anderen Formen der, Mendelscher Regelmäßigkeit folgenden, Bildung synthetischer Neuheiten, seit mehr oder weniger lange voneinander weggespaltene Anlagefaktoren eines entfernten Aszendenten sich im Deszendenten wieder zusammengefunden haben.

Auch an die analytischen Neuheiten, die infolge Mendelscher Spaltung entstehen, sei erinnert.

Es ist uns unmöglich, den Vorgang, der nach Mendelschen Proportionen durch Analyse von Erbkomplexen und Syn- 
these von Erbelementen zu analytischen und synthetischen Variationen führt, vom Mendelschen Vererbungsvorgange begrifflich zu trennen. Die Produkte dieses Vorganges können wir daher wohl sehr vielfach auch nicht als echte Nova bezeichnen.

Es besteht wohl kaum ein $Z$ weifel mehr, daß bei den weiteren Fortschritten der experimentellen Vererbungslehre ein großer Teil der in der freien Natur beobachteten Variationen von ihrem Range als echter Nova abgesetzt und lediglich als Mendelsche Zerfallsprodukte von Erbeinheitskomplexen oder als Zusammenfassung von Erbeinheiten erkannt werden dürften.

Womit gesagt ist, daß solche Mendelschen Neuheiten zweifellos am Zustandekommen der ungeheuren Mannigfaltigkeit in der freien Natur stark beteiligt sind, ja daß das, was wir Evolution nennen, zu einem großen Teil nur einem Gradwechsel in der Kompliziertheit schon dagewesenerVerbind ungen der Erbeinheiten zu verdanken ist, wenn es auch recht wahrscheinlich sein dürfte, daß die Bildung echter, noch nie dagewesener Nova auf diesem Wege nicht selten ist.

Konstanz im Wechsel können wir somit diesem großartigen Vererbungsvorgange zuschreiben, welcher bewirkt, da $\beta$ von Generation zu Generation teils in kontinuierlicher Abstammung, teils Generationen überspringend, dieselben Gruppen äußerer Erscheinungsformen immer wieder auftauchen, und zwar auf Grund von Neukombinationen der Erbelemente, verhältnismäßig unabhängig von äußeren Einwirkungen.

Wirkliche Nova können nun auf verschiedenem Wege entstehen.

Im Anschluß an das Obengesagte soll betont werden, daß kein Grund vorliegt, in Abrede zu stellen, daß durch Neukombinierung von Erbelementen auch zahlreiche echte Neuheiten entstehen können (amphimiktische Neuheiten). In Wirklichkeit ist dies aber, wie aus den obigen Ausführungen ohne weiteres ersichtlich, nicht selten recht schwer mit Sicherheit zu erweisen, weil wir die Geschichte der Erbkombinationen nur mangelhaft kennen. Vielleicht beruht teilweise auf dieser Genese das Vorkommen gewisser Entarteter in Familien, wo man glaubt, weit und breit keine krankhaft hereditären Elemente und keine keimverderbenden Einflüsse wahrnehmen zu können und wo man daher glaubt annehmen zu müssen, daß die Keimplasmen der Eltern „nicht zusammenpaßten“. Denn daß abnorme Kreuzungsprodukte auf diesem Wege entstehen können, beweisen gewisse, mit bekannten Erbformeln versehene, aber häufig unfruchtbare Bastarde.

Die Sicherheit, mit der die Taxierung einer Variation als Neuheit geschehen kann, wächst mit der Kenntnis der Vorfahren und eventueller umgebender Einflüsse und ihrer Auswirkungen, bzw. ihres Fehlens. 
Die Neuheiten, die so entstehen, gehorchen den verschiedenen Modalitäten der Vererbung, zum Teil lassen sie sich bestimmten Regeln noch nicht unterwerfen.

Krankhafte Neuheiten beim Menschen entstehen zumeist wohl durch eingreifende äußere Einflüsse auf den Körper, welche sein Soma verändern, wie Gifte, Krankheitserreger usw.

Dringen die Schädigungen nicht bis zu den Keimzellen vor, um sie irgendwie zu verändern, so haben wir es mit nichterblichen Variationen, mit Somationen (Plate) zu tun.

Es ist eine der wichtigsten Aufgaben der Familienforschung, das Vorliegen solcher Art Neuerscheinungen in jedem Spezialfalle möglichst genau festzustellen, bzw. durch genaue Verfolgung der Nachkommenschaft dieser als Somationen aufgefaßten Variationen, durch Vergleich ihrer Nachkommenschaft mit derjenigen von Eltern, die von dieser Störung frei sind, sowie durch Heranziehung des Tierexperimentes zu beweisen, daß es sich im vorliegenden Falle wirklich nur um Veränderungen des Somas handelt, nicht auch um Veränderungen des Keimes. Denn über die möglichen Wirkungen der verschiedensten Krankheiten auf die Nachkommenschaft wissen wir noch gar nichts Sicheres.

Denn dringen die Noxen vom Milieu im weiteren Sinne (also auch von den Somazellen, die die Keimzellen umschließen) aus bis zu den Keimzellen, sei es des Erwachsenen, sei es des Fötus vor, so können erbliche Variationen entstehen.

Wissen wir auch nicht viel über die Art der Wirkung, so dürfen wir uns vielleicht doch vorstellen, daß die Noxen eine oder mehrere Erbeinheiten in ihrer chemischen Zusammensetzung verändern und schädigen, sie eventuell vernichten, so daß dadurch eine Modifikation der von ihr oder ihnen ausgelösten Eigenschaften bedingt wird, oder ihr normales Widerspiel mit den ungeschädigten Erbeinheiten im ontogenetischen Entwicklungsgange gestört wird.

Hiermit sind wir, wie schon angedeutet, an dem Punkte angelangt, wo neben der Vererbungsforschung eine der wichtigsten A ufgaben der Familienforschung begründet liegt.

Welche Krankheiten, welche Vergiftungen, welche wissenschaftlich feststellbaren Vorgänge im Soma der Eltern setzen krankhafte Anlagen in den Nachkommen? Gibt es überhaupt solche Krankheiten, in welchem Stadium der Krankheit beginnen die Keimeinwirkungen oder hören sie auf, solche Wirkungen zu setzen und sind die derart in den Nachkommen gesetzten Wirkungen tatsächlich erblich, so daß sie unsere Voraussetzungen bestätigen, daß Schädigungen, die durch den Keim geschehen, erbliche Störungen setzen, also erbliche Neuheiten, erbliche Entartung? 
Ferner: Bedingt vielleicht die Individualentwicklung des Somas mit den damit verknüpften chemischen Veränderungen im Stoffwechsel der Eltern (innere Sekretion usw.) eine Beeinflussung der Keimstoffe derart, daß auch ihnen das Optimum der Vitalität nur in jener mittleren Entwicklungszeit beschert ist, in welcher auch das Soma der Eltern im Vollbesitz der Leistungsfähigkeit steht?

Sind also die Erbeinheiten in den Gameten allzu junger und allzu alter Eltern derart minderwertig, daß dies auch in der Nachkommenschaft in dieser oder jener Weise zum Ausdruck kommt? Und sind diese so in den Nachkommen gesetzten Schäden wirklich erblich, selbst wenn die Nachkommen all den bisher genannten keimbeeinflussenden Schäden selbst nicht mehr unterworfen sind? Welchem Typus der Vererbung folgen alle diese Defekte und krankhaften Anlagen, welche durch Schädigungen der Gameten im elterlichen Organismus entstanden sind?

Wenn wir wahre Ursachenforschung treiben ist es stets wichtig zu erforschen, ob eine Abnormität ererbt ist oder neu entstanden, da von dieser Trennung die Begehung zweier ganz verschiedener Wege der Proph ylaxe abhängt, auf der in der Psychiatrie ja doch fast unsere ganzen therapeutischen Hoffnungen aufgebaut sind.

Hält man für ererbt, was durch Keimschäden usw. erworben ist, so wird keine, auch noch so fein erdachte und willensstarke Vererbungsprophylaxe die betr. Abnormität aus der Welt schaffen, denn sie entsteht ja eben durch Fortdauer des äußeren Schadens, dessen Wirkung man verkennt, immer wieder neu.

Hält man aber für erworben, was vererbt ist, so wird die beste Keimhygiene nichts daran ändern, daß das Übel, eben durch Vererbung, immer wieder hervortritt.

Daher der ganz ungeheure, auch praktische Wert der Unterscheidung von Vererbung und Neuschöpfung und die Forderung, sich mit der letzteren gleich intensiv wie mit der ersteren zu befassen.

Die Neuerscheinungen, die die Erkrankung der Eltern an Syphilis an den Nachkommen hervorrufen kann, sind zu einem großen Teil bekannt. Die auffälligsten, die dem klinischen Bilde der kongenitalen Syphilis zugehören, brauche ich nicht zu erwähnen.

Allein es gibt zahllose Syphilitikerkinder, die syphilitische Zeichen nicht mehr darbieten, in deren Blut auch die Wassermannsche Reaktion nicht nachzuweisen ist, die aber doch defekt, oder in auffallender Weise, mehr als Kinder anderer Eltern, mit Krankheiten irgendwelcher Art oder krankhaften Anlagen behaftet sind. Ich erinnere an die Erfahrungen vieler Kliniker in dieser Hinsicht.

Wie außerordentlich wichtig wäre es für diese, ihrem äußeren klini- 
schen Gepräge nach gewissen erblichen Formen nah verwandten, häufigen Krankheits- und Defektzustände, die man bisher so gerne stets nur auf die „Erblichkeit", auf die „Belastung“ zurückführte, als wirkliche Neuerscheinungen syphilitischer Genese mit Hilfe der Familienforschung nachzuweisen.

Allein, die syphilitische Genese dieser Formen als erwiesen angenommen, sind diese Neuerscheinungen im Nachkommen des Syphilitikers erblich, $d . h$. übertragen sie sich, und wenn ja, nach welchen Vererbungsregeln, auf deren Nachkommen? Sind sie dominant und damit ,unauffrischbar"? Sind sie recessiv oder intermediär gehend und damit „regenerierbar“. Wer wird hier nicht auf den Gedanken kommen, daß zahllose Formen von Dummheit, Beschränktheit, Debilität, Nervosität, Neurasthenie und Psychopathie und ganz leichte Formen von Psychosen (Cyclothymien usw.), mit denen sich große Familien, mit denen sich unsere Völker weiterschleppen, ursprünglich auf diese Weise erstmals entstanden sein können? Und wer wird, nach dieser berechtigten Mutmaßung, nicht das Bedürfnis empfinden, an Hand der Struktur der Familien durch Vergleich der Strukturteile, welche vor, mit denen, welche hinter dem Einsetzen des verdächtigten Schadens (Syphilis) liegen, sowie durch Vergleich von gleichstammigen Generationsfolgen, welche syphilisfrei mit solchen, wo Syphilis an einem Punkte eingefallen ist, diesen hochwichtigen Zusammenhang der Dinge, also diese erstmalige Entstehung einer von dann ab erblichen Abnormität, auch zu beweisen?

$\mathrm{Zu}$ leicht stelle man sich diesen Beweis freilich nicht vor, denn nur genaue klinische Determinierung der vor und nach Einsetzen der Syphilis in dem betreffenden Stamme konstatierten Störungen und ein gewissenhafter Proportionsvergleich wird uns in die Lage versetzen, Erbwirkungen von Gliedern aus auszuschließen, welche über der syphilisaffizierten Generation liegen.

Mit dem Problem der Beschaffenheit der Syphilitikerkinder hängt dasjenige von der Qualität der Paralytikerkinder eng zusammen.

Zunächst werden diese Kinder denselben oder doch analogen Veränderungen unterworfen sein, wie die Kinder gewöhnlicher Syphilitiker, wenn dies auch durchaus nicht sicher ist, da es nicht ausgemacht ist, daß die Syphilis in einem paralyse-prädisponierten Individuum dieselben Körperreaktionen und dieselben keimschädigenden Wirkungen entfaltet, wie in einem von dieser Disposition freien Menschen. Wir haben also die Paralytikerkinder der präparalytischen oder syphilitischen Periode des Paralytikers zu unterscheiden von den Kindern jener Periode, in der im Organismus des Vaters die entstehende Paralyse ihre eigenen Wirkungen auf Organismus und Keim mutmaßlich zu entfalten beginnt. Das wären die eigentlichen Paralytikerkinder. 
Ja in günstigen Fällen, wo die Infektion später in der Ehe erfolgte, werden in der Gesamtkinderserie noch präsyphilitische Kinder theoretisch zu unterscheiden sein.

Es ist klar, wie verwickelt da die Qualitätskurve werden muß, wenn sie unserem Verdacht entsprechend verläuft. Von den Präsyphilitikerkindern müßte zu den Syphilitikerkindern ein jäher Qualitätsabfall (Aborte usw.) stattfinden, mit, im klassischen Falle, allmählicher Erholung zu Totgeburten, Kongenital-Luetischen, anderweitig Abnormen, Gesunden, um schließlich, mit Einsetzen des Abschnittes der eigentlichen Paralytikerkinder wieder einer allmählichen, der Art nach freilich noch ganz unbekannten Verschlechterung der Geburten Platz $\mathrm{zu}$ machen.

Auch hier wäre natürlich zu unterscheiden zwischen Nachkommenschaft männlicher und solcher weiblicher Paralytiker, da ja die Verschlechterung der Kinder der paralytischen Periode eine rein mütterlichparalytische Wirkung auf den Fötus darstellen, der Keim des paralytischen Vaters aber unbeschädigt bleiben könnte.

Auch hier leuchten die theoretischen wie praktischen Konsequenzen, welche eine wissenschaftliche Rassenhygiene zu ziehen hätte, ein.

Noch bedeutend schwieriger liegen die Dinge beim Alkoholis mus als Keimverderber.

Hier stehen sich zwei Meinungen gegenüber. Die eine will, daß der Alkoholmißbrauch der Eltern an und für sich zu abnormer Nachkommenschaft führt.

Die andere macht geltend, daß die Nachkommen nur dann abnorm sind, wenn der trinkende Elter oder dessen Erbplasma selbst abnorm ist.

Bei der enormen Verbreitung des Alkoholismus und dem Energieaufwand, der sowohl zur Produktion als auch zur Bekämpfung gemacht wird, kann es nicht gleichgültig sein, wo hier die Wahrheit liegt.

Auch hier wird man, wie im Falle der Syphilis, vorgehen müssen, wobei aber von vornherein aus der psychiatrischen Erfahrung feststeht, daß jedenfalls mit echt erblichen Fortwirkungen in der Nachkommenschaft der Trinker zu rechnen ist, da jeder Unterrichtete weiß, daß fast alle Alkoholiker, wenigstens die, die in Anstalten kommen, selbst vor Beginn des Mißbrauchs abnorm veranlagt sind oder wenigstens außerordentlich häufig aus schwer belasteten Familien stammen.

Man wird dann die klinischen Formen der Alkoholikernachkommen genau vergleichen müssen mit denen voriger Generationen und kollateraler Generationen, man wird sich fragen müssen, ob auch die Proportionen durch die Annahme reiner Vererbungswirkung nicht erklärt zu werden vermögen. Man wird die körperlichen und geistigen Mißbildungen und Krankheitsanlagen, die Sterblichkeit usw. der Nachkommen vergleichen mit den Produkten der Alkoholzeugung im Tierversuch, 
der als echter Keimverderbversuch (nur Vater zu vergiften!) allerdings erst noch gemacht werden muß. Besonders lehrreich aber sind die Naturexperimente, wie sie durch mehrfache fruchtbare Heiraten, sowohl der nüchternen Mutter als des trinkenden Vaters oder umgekehrt gegeben sind und wo die reinen Alkoholwirkungen auf die Nachkommenschaft, abzüglich auch hier wieder von Bastardierungswirkungen, die auf Vererbung zurückzuführen sind, sich doch mehr oder weniger isoliert herauszuschälen versprechen. In dieser Hinsicht wäre es speziell von besonderem Wert, wenn in der Annahme, daß der fortschreitende oder auf gleicher Höhe der Unmäßigkeit sich haltende Alkoholgenuß des Elters allmählich kumulierende Wirkungen auf die Keime, also auch Früchte, entfalten dürfte, erwiesen werden könnte, daß tatsächlich (wie manche Franzosen behaupten, was aber Orschansky bestreitet) die Kinder der Alkoholiker im Laufe der Zeugungsperiode schlechter werden. Analog wie Scholten eine zunehmende Verschlechterung der Paralytikerkinder mit fortschreitender Einwirkung des Paralysegiftes auf die Keime annimmt.

Oder wenn erwiesen werden könnte, daß umgekehrt die Kinder, welche in die Zeugungsperiode des geretteten Trinkers fallen, bezüglich der Mortalität und qualitativen und quantitativen Morbidität besser geraten als diejenigen, die in seine frühere Zeit des Alkoholmißbrauches fallen.

An einem großen, kritisch ausgelesenen Material von Mitgliedern von Vereinen, die sich schon seit vielen Jahren der Trinkerrettung widmen, müßte diese Frage zu entscheiden sein.

Ferner werden am trinkenden Elter Perioden der Trunksucht und Nüchternheit mit den Produkten der zugehörigen fruchtbaren Zeugungen in Vergleich zu ziehen sein.

Würde sich auf diesem Wege einwandfrei erweisen lassen, daß Alkoholmißbrauch des Vaters tatsächlich zur Entartung der Nachkommen führt, so wäre damit auch der meines Wissens noch nicht erbrachte, aber theoretisch wie praktisch außerordentlich wichtige Beweis geliefert, daß tatsächlich, nicht bloß wie, allerdings mit sehr viel Berechtigung, angenommen wird, der Alkohol die Gameten schädigt. Wäre das selbst für das Tier erwiesen (was es, wie gesagt, noch nicht ist), so würde dieser Nachweis für den Menschen durchaus selbständige Bedeutung besitzen, da ein Tierstamm, der nie Alkohol aufnahm, ganz anders reagieren kann wie ein Menschenstamm, in dem sich durch generationenlangen Genuß des Giftes schon eine gewisse Einstellung, wenn man will Anpassung, vollzogen haben kann.

Trinkende Mütter eignen sich zu diesem Beweise nicht, da bei ihnen ja auch der sich entwickelnde Embryo vom Alkohol beeinflußt wird, Embryoschädigungen durch Alkohol aber eine durchaus mannigfach 
bewiesene Tatsache sind. Aber auch sicher konstatierte Embryonalschädigungen (wo die Zeugungen also unter den günstigsten, die Schwangerschaft unter den ungünstigsten Bedingungen verlaufen ist) können zum Ausgangspunkt praktisch wie theoretisch hochwichtiger Untersuchungen gemacht werden, indem die erbliche Fixierbarkeit des Resultats bestimmter Embryonaleinwirkungen (z. B. Alkohol, Blei usw.) zu untersuchen wäre. Denn es kann nicht gleichgültig sein, ob erst dem fertigen Organismus oder auch schon dem werdenden Individuum (Embryo) die Fähigkeit des Erwerbes (auf dem Umwege der Keimschädigung entstandener und dann) vererbbarer Eigenschaften zukommt oder nicht.

Wo eingreifende, keimverderbende Wirkungen anderer Gifte und anderer Krankheiten (z. B. Blei, Malaria usw.) vermutet werden, müssen sie in derselben Art, mit derselben sorgfältigen Rücksichtnahme auf die eventuelle Mitwirkung erblicher Überlieferung erwiesen werden.

Eine Untersuchung über die aus Keimverderbnis hervorgegangenen Nachkommen muß nach der zweifachen Richtung, nämlich ob sie sich nach den Gesetzen eines in der Familie vorhandenen Erbübels, oder nach eigenen, durch ihre blastophthore Entstehung gegebenen Regeln vererben, stets angeschlossen werden.

Besonders verwickelt gestaltet sich der Nachweis des Zusammenhanges zwischen absolutem Zeugungsalter der Eltern und Qualität der Nachkommenschaft. Er ist aber wiederum so außerordentlich wichtig, weil es sich auch hier, wie im Falle der Syphilis und des Alkohols um beherrschbare, verhältnismäßig leicht abstellbare Ursachen pathologischer Keimvariationen handelt, also um Vorarbeiten von eminent praktischer prophylaktischer Bedeutung.

Zur Untersuchung ist ein besonders großes Material erforderlich und, wie ich betonen möchte, eines, das nicht bloß das Zeugungsalter der Eltern und die Geburtsdaten und den Gesundheitszustand der Kinder, sondern auch alles das ebenfalls aufweist, was als Ursache von Entartung oder als Ausgangspunkt erblicher Überlieferung gelten muß oder vermutet wird. Denn wo ein krankhaftes Merkmal nach Art und Proportion seines Vorkommens in einer Generation die Gesetze erfüllt, die ihm durch das nachgewiesene gesetzmäßige Vorkommen desselben Merkmals bei den Aszendenten, Kollateralen und Deszendenten vorgeschrieben sind, dürfte es vom ätiologischen Gesichtspunkte aus, mit Bezug auf dieses spezielle Merkmal wohlverstanden, gleichgültig sein, ob sein Träger ein Jünglings- oder ein Greisenkind ist.

(Doch komme ich auf beachtenswerte abweichende Ansichten, die darüber geäußert worden sind [Orschansky] oder gehegt werden [Schweighofer] noch zurück.) 
Auch dürfte es in Fällen einer Schwer-Trinker-Deszendenz, wie ich sie beobachtet habe, wo sämtliche Kinder an Krämpfen gestorben sind, mit Ausnahme eines einzigen, welches dieselben Krämpfe hatte, aber sie überstand und seine Rettung mit dem Zurückbleiben schwerer geistiger und körperlicher Entartung bezahlte, zur Erklärung der ganzen Art dieser Deszendenz in ätiologischer Beziehung verhältnismäßig gleichgültig sein, ob der Defekt in eine pessimale oder in die optimale Zeugungsperiode des Vaters fällt, wenn auch die Vermutung nahe liegt, daß der letztere Fall am Davonkommen dieses defekten U̇berlebenden ursächlich mitbeteiligt sein könnte.

Auch wird man in einer Syphilitikerdeszendenz es nach allen Erfahrungen ganz selbstverständlich, d. h. mit der Heilungstendenz der Syphilis zusammenhängend, finden, wenn gerade die ersten Früchte des Syphilitikers schlechter, die mittleren und letzten besser sind und bei so gearteter Struktur der Deszendenz kaum Veranlassung nehmen, sie ätiologisch etwa mit einer Altersevolution der elterlichen Gameten in Zusammenhang zu bringen. Auch wo zehrende oder andere schwere Krankheiten zufällig in Zeugungsperioden fallen, denen man als solchen eine für die Nachkommen qualitätsmindernde Immanenz beimessen will, muß die Möglichkeit, daß von diesen Krankheitsvorgängen Einwirkungen auf die Deszendenz ausgehen, in ernste Berücksichtigung gezogen werden. Nach all diesen und vielen anderen möglichen Ursachen mehr muß sonach das auf die Beziehung des Zeugungsalters der Eltern zur Entartung der Nachkommenschaft hin statistisch bearbeitete Material aufgesplittert werden, damit die reine Wirkung der Altersevolution der Keime gesondert hervortritt. Jedenfalls ist die Lösung der Frage, ob in der Nachkommenschaft durch Unreife oder durch Altersaufbrauch (Marro, Schweighofer) der elterlichen Keime abnorme Zustände des Nervensystems entstehen können, unserer höchsten Anstrengung wert, insbesondere aber die Lösung der Frage, ob dadurch wirkliche Neuerscheinungen entstehen können (mögliche Beeinflussung des Erbganges durch Keimunreife und Aufbrauch, s. unten) und ob sie in einer regelmäßigen Weise mit entsprechenden Auswirkungen auf körperlichem Gebiete verknüpft sind. $\mathrm{Zu}$ den statistischen Untersuchungen selbst $\mathrm{muß}$ das genaue Zeugungsalter der Eltern (Geburtstag der Eltern) und die genaue Reihenfolge der Geburten (Geburtstag der Kinder), sowie bei allen Deszendenten das Alter, in dem die Erkrankung beginnt, bekannt sein.

Bei manchen Erkrankungen, auch Psychosen, zeigt sich erfahrungsgemäß bald, daß die Erstgeborenen mehr betroffen sind als die später Geborenen. (Natürlich ist die Häufigkeit der Erkrankung jeder Geburtennummer in Prozenten sämtlicher gleicher Geburtennummern zu berechnen.) Dieser Umstand deutet an und für sich auf junge Eltern- 
zeugungen. Allein er könnte, und das scheint der Fall zu sein, zum Teil auch darauf beruhen, daß die Erstgeborenen eine geringere Sterblichkeit als die anderen Geburtennummern, namentlich die höchsten, aufweisen bis zu dem Alter, wo die der Untersuchung unterliegende Krankheit überhaupt erst beginnt, also konstatierbar ist, so daß gleiche, spezifische Morbidität aller Geburtsnummern an und für sich vorausgesetzt, es begreiflich ist, wenn der Prozentsatz der aus dem genannten Grunde Erkrankenden unter den Erstgeborenen größer ist als in den Geburtennummerkategorien mit höherer präpsychotischer Sterblichkeit.

$\mathrm{Zu}$ untersuchen ist selbstverständlich auch, ob die Überschreitung der erwarteten Krankheitsziffer nicht vielleicht von einer großen Unehelichkeit herrühren kann. Denn die meisten Unehelichen sind Erstgeborene, während sich unter den anderen Geburtennummerkategorien sehr wenige befinden. Sie sind wiederum einer Reihe besonderer, zum Teil unkontrollierbarer (z. B. Erblichkeit!!) Einflüsse unterworfen, so daß man sie am besten zu diesem Zweck eliminiert, $d$. h. gesondert behandelt.

Auch ist es von Bedeutung, wie das Material aus kinderreichen und kinderarmen Familien zusammengesetzt ist.

Überwiegen die kinderarmen, und sind diese Familien kinderarm infolge frühen Todes der Eltern oder infolge Zeugungs- oder Befruchtungsunfähigkeit der Eltern, so kann ein Teil des Erkrankungsauftaktes der Erstgeborenen mehr auf Krankheit der Eltern, als auf deren Jugend zurückzuführen sein. Auch die Frage, ob die Mehrerkrankung der Erstgeborenen vielleicht mit der größeren erblichen Belastung zusammenhängt, kann statistisch nachgewiesen werden, wenn auch die weiter oben zur Lösung der Erblichkeitsfrage angegebenen Mittel weitaus gründlicher sind. Sind alle diese Möglichkeiten durch statistische Gruppierungen eines medizinisch gut determinierten Materials ausgemerzt, so kann die Wirkung des Zeugungsalters rein hervortreten, und zwar um so deutlicher, wenn man das Material trennt in eines, wo beide Eltern alt oder jung, oder der eine von beiden sehr jung, der andere sehr alt, oder wo einer im optimalen, der andere im extremen Alter sich befindet.

Doch kommt, bei alten Müttern besonders, wohl auch die Gesamtgeburtenleistung, also die eventuelle Geburtserschöpfung in Frage, welche ebenfalls durch geeignete statistische Gruppierungen auszuschalten ist. Auch das Geburtenintervall, die Stillungsdauer und anderes mehr ist zu berücksichtigen.

Am schwierigsten, und erst nach Lösung aller anderen Fragen zu beantworten ist die Frage, ob die Erstgeburtsstellung als solche eine Prädisposition zu erhöhter Erkrankung schafft, da die Frage aufgeworfen werden kann, ob nicht die Erstgeburt vielfach das Opfer der allerersten Ingangsetzung des Geburtsmechanismus sein könnte. 
Es wird sich so, bei Befolgung der im obigen vorgeschlagenen zahlreichen gleichzeitigen Kontrolluntersuchungen über andere mögliche Ursachen in exakter Weise zeigen lassen, ob ,schwere Geburt ", und die Erstgeburtsschwierigkeit im besonderen, wirklich die enorme Bedeutung spielt, die ihr von früheren Psychiatern namentlich (auch jetzt spielt sie wenigstens in der Anamnesenaufnahme noch eine große Rolle) vielfach zugemessen wird.

Auf dieselbe Art der getrennten statistischen Behandlung der möglichen ursächlichen Faktoren ist die Frage zu behandeln, worauf es beruht, daß S pätgeborene, also höhere Geburtennummern und Letztgeborene, ob sie höheren oder niederen Nummern in der Geburtenreihe entsprechen oder nicht, eine größere Morbidität für bestimmte Affektionen besitzen oder zu besitzen scheinen, als mittlere Geburtennummern.

All diese ätiologischen Untersuchungen sind angesichts des mit steigendem Wohlstand heraufgerückten Heiratsalters und in Anbetracht der zunehmenden künstlichen Einschränkung der Fruchtbarkeit bei den höchsten Kulturvölkern für die Prozentverschiebung zwischen gesund und krank der eingeschränkten Generation und für die entsprechende prozentuale Zusammensetzung aller folgenden Generationen, also für ihre gesundheitliche Zukunft und für ihre numerische Kraft von so ungeheurer Bedeutung, daß wir ihrer exakten Lösung nicht genug Arbeitseifer und nicht genug wissenschaftliche Genauigkeit zuwenden können.

Mit der Untersuchung der Folgen des absoluten Zeugungsalters kann eine weitere über die pathogene Bedeutung des relativen Zeugungsalters, also des Altersunterschiedes der Eltern, verbunden werden, indem die entsprechenden Kinderserien zusammengruppiert und miteinander verglichen werden, da es nicht ausgeschlossen ist, daß auch hier Differenzen in den Folgen für die Nachkommenschaft bestehen.

Es ist denkbar, daß große Differenzen im Alter der Zeuger an und für sich, abgesehen von den durch obige Untersuchungen isolierten Ursachenfaktoren, ungünstigere Keimkombinationen ergeben, und daß es ein Optimum der Altersdifferenz für die Zeugung gibt.

Wichtige Untersuchungen über die Ursachen von Neuerscheinungen betreffen die Geburtenintervalle, die vor den Probanden und speziell auch zwischen dem erkrankten Probanden und der vorhergehenden Geburt liegen.

Schon lange vermutet man, da $B$ rasch aufeinanderfolgende Geburten schädlich, nicht bloß für die Mutter, wo man das weiß, sondern auch für die Vitalität der Früchte selbst sind.

Seit Westergaards Untersuchungen weiß man das bestimmt für die erhöhte Sterblichkeit. Allein sehr vieles spricht dafür, daß auch 
in Hinsicht auf die Morbidität von Opfern zu kurzer Geburtsintervalle gesprochen werden kann.

Auch hier ist nicht von vornherein einzusehen, wieso eine erhöhte Erkrankungsziffer bei zu kleinen (aber auch zu großen!) Geburtenintervallen mit echter Erblichkeit zusammenhängen soll.

Ist das Vorliegen dieses mit den oft genannten, genealogischen Mitteln zu erweisenden Zusammenhanges also ausgeschlossen und sind andere klinisch oder statistisch konstatierbare Phänomene als Ursachen sorgfältig ausgemerzt, so haben wir hier wiederum einen beherrschbaren Erzeuger pathologischer Variation von größter praktischer Bedeutung vor uns, der wiederum, wie in den obigen Fällen, Ausgangspunkt einer bestimmten pathologischen Erbfolge werden kann (was aber eigens, ohne langes Theoretisieren untersucht werden muß), da man nicht weiß (ganz ähnlich wie im Falle der Embryonalvergiftung durch mütterlichen Alkoholmißbrauch), ob nicht die Schäden, die ganz offenbar in utero gesetzt worden sein müssen, nicht auch bis zum Keim des Embryo vorgedrungen sind und ihn dauernd geschädigt haben, womit der Ausgangspunkt für eine krankhafte Erbquelle gegeben sein kann.

Hochinteressant, allerdings nur an einem sehr großen Material lösbar, ist auch das Problem, ob die in bestimmten Monaten erfolg te Zeugung an und für sich einen erhöhenden oder erniedrigenden Einfluß auf die Vitalität und somit Mortalität und Morbidität der Frucht haben kann.

Die Frage, die mit dem Problem der Brunstzeit beim Menschen und der Frage der optimalen Aufbringungszeit für den Säugling usw. eng zusammenhängt, wäre sicher jetzt schon zu lösen, wenn wir anstatt der heutigen amtlichen Statistik, deren Einheiten außerordentlich qualitätlos sind, eine ausgedehnte Familienstatistik mit allseitiger Qualifizierung der statistischen Einheiten hätten.

Es ist klar, daß die Antwort auf diese Frage für uns wichtig sein muß, da auch die Wahl der Zeugungszeit in hohem Maße in unser Belieben gestellt ist, also wiederum eventuell eine beherrschbare Variationsursache darstellen würde.

Schließlich kann die Familienforschung mehr Klarheit in die Frage der psychoseerzeugenden Wirkung des Milie us und der allge meinen Lebe nsweise bringen, der Einwirkungen großstädtischer Bedingungen, schlechter, giftiger Atmosphäre, Mangel an Bewegung, U̇berernährung, Unterernährung, Reizüberschüttung und chronischer Affektladung, hoher Verantwortung, Berufsschäden, einseitiger geistiger Differenzierung usw., kurz in die hochbedeutsame und aktuelle Frage der entartenden Wirkung des Großstadtlebens, der Domestikation, der Kultur in der Gesamtwirkung und in der Wirkung der ihr Wesen aus- 
machenden oder sie nur begleitenden Einzelfaktoren. Und zwar deshalb, weil eben das Familienmaterial, dessen wir uns zur Untersuchung dieser Spezialbeziehungen bedienen, allseitig ätiologisch in jedem Glied genau und speziell determiniert ist (mit Bezug auf Erblichkeit, offenkundige Keimschäden, Alterszusammensetzung usw.), weshalb wir das Vorliegen bestimmter, verdächtiger, ätiologischer Momente statistisch ausscheiden und zur klareren Erkenntnis für die Wirkung anderer Faktoren kommen können, was bei einer gewöhnlichen statistischen Masse, deren Einheiten mit Bezug auf die meisten biologisch wichtigen Daten ganz unbekannt sind, zumeist unmöglich ist.

Die Familienforschung ist in der umfassenden Extensität und Intensität, wie wir sie verstehen, wie keine andere Methode außer dem Experiment geeignet, die bei der Erzeugung von Krankheit und Entartung miteinander konkurrierenden Ursachen voneinander $\mathrm{zu}$ isolieren und so in Art und Stärke ihrer Einzelwirkung zu erkennen.

Auch die wahren Wirkungen von Erziehung und Beruf können mit Sicherheit nur ermessen werden, wenn wir aus der genauen Kenntnis der Familienstruktur wissen, daß andere Ursachen nicht mitspielen können.

Die Familienforschung wird für die vagen und sich oft diametral entgegenlaufenden Ansichten, die über die Wechselwirkungen von Kultur und Entartung herrschen, durch genau begründete Zurückführungen auf bestimmte ursächliche Faktoren die exakten wissenschaftlichen Grundlagen erst zu schaffen vermögen.

Wie auf klinischem Gebiet die Vorhersage der Prognose, so hat auf genealogischem Gebiete das Problem der generativen Prognosebestimmung, die Zeugungsprognose (einschl. der Vererbungsprognose) für einen gegebenen Probanden ungeheure Bedeutung.

Sie baut sich auf auf der genauen Kenntnis der pathologischen Erbfolge im Stamme, auf der Qualität des Probanden selbst, seinem Alter, seinem Geschlecht, seinem Rassetypus und aus den vorhandenen oder vorausgesetzten äußeren Milieufaktoren und entsprechenden Prognosebestimmern beim Partner und dessen Familie.

Es dreht sich somit alles darum, sich durch die Forschung der Beantwortung der kompliziertesten aller Fragen allmählich immer mehr zu nähern, unter welchen genealogischen und individuellen wahrnehmbaren oder erschließbaren oder in der Zukunft als eintretend vorausgesetzten Vorbedingungen die verschiedensten vererbbaren krankhaften Anlagen bei einem gegebenen Probandenpaar sich nicht bloß vererben können, sondern müssen, bzw. nicht können, und unter welchen analogen Bedingungen krankhafte Anlagen nicht ererbter Genese unter der Nachkommenschaft auftreten müssen, bzw. nicht können. 
Im vorhergehenden wurde, zum Zwecke der Gewinnung einer Utbersicht über die mannigfaltigen Auswirkungen der Naturkräfte, absichtlich in solche unterschieden, welche das treibende Moment beim konservativen Festhalten (eben bei der erblichen Überlieferung) der Formen bilden und in solche, welche diese Konservativität stören.

Und auch bei den Formen selbst, den Resultaten dieser zwei verschiedenen Kräfte, wurde unterschieden in schon dagewesene, vom äußeren Milieu verhältnismäßig unabhängige, eben ererbte und vererbende (im weitesten Sinne) einerseits und nichtererbte, d. h. neue Merkmale und Merkmalskomplexe andererseits, welche wiederum vererben können, dies aber nicht müssen.

In Wirklichkeit liegen die Vorgänge und die Formen aber wohl kaum so schön getrennt voneinander, wie in der begrifflichen Abstraktion. Ja, vieles spricht für eine rege Wechselwirkung der beiden Vorgänge, des konservativen und des neuschöpfenden, a ufeinander.

$\mathrm{DaB}$ unsere Forschung, zwar geleitet und angeregt durch theoretische Erwägungen, aber nicht blind auf sie vertrauend, darauf gerichtet sein muß, zu ergründen, welche Neuschöpfungen erblich sind, welche nicht und wie und ob sie gesetzmäßig vererben, habe ich schon betont.

Es bleibt uns somit nur noch die hochwichtige Frage zu erörtern, ob es eine erkennbare und auf Gesetzmäßgkeiten zurück. führbare Beeinflussung der Verbindung von intakten Erbfaktoren und der Latenz und äußerlichen Manifestation der einzelnen intakten Erbeinheiten, sowie bei der Kreuzung sich verbindenden Summen von Erbfaktoren durch Einflüsse des äußeren Milieus gibt.

Auch hier gibt uns das Tier- und Pflanzenexperiment Anregungen, und das Tempo seines Fortschrittes verschafft uns Gewähr dafür, daß es unsere Untersuchungen am Menschen auch in Zukunft zu leiten bestimmt ist.

Man hat gesehen, daß bei verschiedenen Tieren durch Einwirkung abnormer Temperaturen, auf das Puppenstadium z. B., Abänderungen am erwachsenen Tier hervorgerufen werden können, welche in einzelnen Fällen bei den Nachkommen auch dann, wenn diese bei gewöhnlicher Temperatur aufgezogen werden, in abgeschwächter Form wieder hervortreten.

Es entstehen „Kälte- und Wärmeaberrationen“, welche in bestimmten Eigenschaften mit natürlichen Varietäten nordischen und südlichen Klimas übereinstimmen.

In einem Teil solcher Temperaturexperimente, namentlich bei geringeren Graden der Einwirkung, treten somit unter normalen Be- 
dingungen in der betreffenden Spezies latente, aber bei anderen Spezies unter normalen Bedingungen regelmäßig zu findende Merkmale auf.

Es wurden durch die Reize künstlich Abänderungen erzielt, die zum Teil erblich sind und als Entdifferenzierungen oder sogar als Rückschläge, als Atavismen, aufzufassen sind.

Allein in anderen Fällen werden in den verschiedenen Spezies außer diesen atavistischen Abänderungen noch andere erzielt, welche weder als Atavismen, noch als Entwicklungshemmungen ontogenetischer Art, noch als phylogenetische Reminiszenzen gedeutet werden können, sondern Bildungen, die anderswo zuvor wohl in den Elementen, aber doch in dieser Kombination nicht zu finden sind und wahrscheinlich (Haecker) als Äu Berungen von in den verschiedenen Spezies noch gemeinsamen, generellen, normalerweise latenten Potenzen zu deuten sind, welche eben erst unter dem Ein. fluß abnormer Reize geweckt wurden und welche unter normalen Bedingungen (wie sie jemals faktisch in der Natur geherrscht haben) nicht hervorgerufen zu werden vermögen.

Diese allseitigen Abänderungen treten, wie schon erwähnt, bei vielen derartigen Versuchen, bei den Nachkommen in qualitativ identischer Form, wenn auch vielfach quantitativ abgeschwächt, wieder hervor, vererben sich also und zwar, wie in den bekannten Experimenten von Kammerer, vielfach nach Mendelscher Regel.

Mit diesen Versuchen kann man gewisse, absolut exakt allerdings noch nicht in all ihrer Abhängigkeit von anderen Phänomenen aufgeklärte Erscheinungen auf dem Gebiet der menschlichen Pathologie in Zusammenhang bringen.

So ist es außerordentlich wahrscheinlich, daß es eine Anzahl durch äußere Schädlichkeiten hervorgerufene Abänderungen beim Menschen gibt, welche nicht in qualitativ gleicher Gestalt bei seinen Nachkommen wieder zum Vorschein kommen, sondern in qualitativ modifizierter Form.

Es sind Gifte, welche derartige, mit den Schäden, die sie im Elter hervorrufen, nichtidentische Wirkungen in seinen Nachkommen entfalten. Besonders, wie wir mit großem Recht stark vermuten, der Alkohol.

Beim Trinker zeigen sich die Wirkungen des Alkoholmißbrauches zumeist in ganz anderer Weise als bei seinen Nachkommen. Von einer eigentlichen Vererbung kann daher nicht die Rede sein.

Sie zeigen sich in Gestalt allgemeiner Körperkleinheit, Körperschwäche, geringer Widerstandsfähigkeit aller Art und in der Neigung zu Krankheiten, auch Psychosen aller Art, kurz in einer krankhaften allgemeinen Abänderung der Reaktionsweise gegenüber den Außenbedingungen. 
Es würde in den meisten Fällen wohl schwer, ja unmöglich sein, diese Dinge auf Erblichkeit in Mendels Sinne und Umfange zurückzuführen (synthetische oder analytische Neuheiten).

Dagegen ist die Erklärung auf der Hand liegend, daß der Alkoholmißbrauch nicht bloß das Soma des trinkenden Elters schwächt und erkranken macht, sondern daß er auch seine Keimzellen schwächt und damit die aus ihm hervorgehende Nachkommenschaft in der oben beschriebenen allgemeinen Form. (Wobei echte Erbwirkungen natürlich noch dazu gefunden werden können, wenn die Erbeinheiten der Verwandten in entsprechender Weise beschaffen und aufgebaut sind.)

Ich habe die damit zusammenhängenden Probleme und Forschungsziele schon oben berührt (S. 553), wo von Einflüssen die Rede war, welche imstande sind, über den Weg einer Keimschädigung krankhafte Neuerscheinungen beim Nachkommen hervorzurufen.

Eine Frage von eminent wichtiger theoretischer und praktischer Bedeutung habe ich aber noch nicht gestreift: Kann der Alkohol als Keimschädiger den Erbgang als solchen, die Kombination der Erbelemente sowohl für gesunde, wie für kranke Anlagen, ihr Latent- und Manifestwerden beeinflussen; vermag er dies für die für die eigene Blutsverwandtschaft des Trinkers selbst in Betracht fallenden Erbfaktoren oder auch für die für seine nüchterne Frau in Betracht fallenden Erbelemente? oder für beide zusammen? Wenn dies der Fall, wie geschieht es, und hat es in gesetzmäßiger Weise statt?

Ist er imstande, das ohne sein Hinzutreten erwartete und aus der genaueren Kenntnis der Blutsverwandtschaft nach Mendel berechnete Resultat des Erbganges irgendwie zu modifizieren, sei es durch Bewirken von Dominanzwechsel für diese oder jene bekannte Erbeinheit, oder durch Reaktivierung von Atavismen oder (siehe oben) ihnen ähnlicher, gewissen Familien oder Stämmen oder Rassen gemeinsamer, genereller, normalerweise aber latenter Potenzen? (Brutalität, Willensschwäche usw.)

Und können diese dann wieder verschwinden in den weiteren Nachkommen oder sich gleichsinnig auf diese vererben? (Analogie siehe oben.)

Beispiele von Dominanzwechsel habe ich oben schon erwähnt.

Es ist ein Phänomen, das bereits als Tatsache von der allergrößten Tragweite ist, auch wenn es sich in seiner Genese noch nicht erklären läßt.

Es liegt aber nicht der leiseste Grund vor, abnorme, schädliche Milieueinflüsse auf den Keim von der Mitwirkung am Zustandekommen dieses Phänomens auszuschließen. 
Im Gegenteil sprechen die Beispiele, die bekannt geworden sind, dafür.

Denn, wenn es richtig ist, daß der Alkoholismus im Elter schwere Psychosen erzeugen oder auslösen kann, im Individuum also Erbelemente und Kombinationen von solchen schlummern, deren somatische Produkte eine so eigenartige Reaktion auf das Gift zulassen, warum soll dann nicht mindestens, in Analogie der Genese der Temperaturaberrationen u. dgl. der Alkohol ebenfalls pathologische Erbanlagen reaktivieren, die seinerzeit bei irgendeinem Vorfahren einmal durch seine direkte oder indirekte Mitwirkung zustande kamen und zum Nachkommen weitergegeben wurden?

Denn wie in einem gegebenen zoologischen Artbild zweifellos viele Eigenschaften unter normalen Bedingungen dauernd schlummern, so tun sie das wohl auch in der menschlichen Rasse oder in einem gegebenen größeren oder kleineren blutsverwandten Stamme, können aber wohl hier ebensogut wie dort durch abnorme Lebensbedingungen, deren Wirkungen bis zu den Geschlechtszellen vordringen, wieder reaktiviert, wieder zum Vorschein gebracht werden.

So gibt es denn manche Forscher, denen Beobachtungen zur Verfügung stehen, aus denen die allerdings noch ziemlich allgemeine Erkenntnis hervorzugehen scheint (sicher ist sie noch nicht), daß der Alkoholmißbrauch eines Individuums ganz besondere Verheerung bezüglich des Auftretens von Psychosen unter der Nachkommenschaft anzurichten scheint, wenn das betreffende trinkende Individuum selbst, oder sein Partner, oder wenn beide belastet sind.

Es werden durch die oben mehrfach besprochenen Vergleiche der Nachkommenschaftsresultate mit und ohne trinkenden Elter usw. die getrennten Wirkungen 1. der echten Erblichkeit, 2. der neuschöpfenden, von Keimverderbnis herrührenden alkoholistischen Nachkommenschaftsmorbidität und 3. der Reaktivierung von schlummernden Erbeinheiten, d. h. von Belastungen der beiden Partner voneinander zu isolieren sein.

Es ist klar, daß solche Erwägungen sich mit Bezug auf alle Gifte, von außen eingeführte, wie im Körper produzierte, anstellen lassen, welchen eine eingreifende, schädigende Wirkung auf den Gesamtorganismus des Menschen (Soma + Geschlechtszellen) zugeschrieben wird, und daß sie auch mit Bezug auf die Reife- und Aufbrauchvorgänge in den Gameten im Verlaufe des Lebensganges eines Menschen (siehe oben), auch auf die besprochenen Faktoren: Geburtenintervall usw. möglicherweise Anwendung finden müssen.

Insbesondere wird hier die Frage zu beantworten sein, ob und wie das absolute und relative Alter eines zeugenden Elters maßgebend 
dafür sein kann, ob in den Nachkommen mehr die Erbmasse seiner eigenen Aszendenz oder mehr diejenige der Aszendenz des Partners oder ob mehr die normale oder mehr die abnorme Erbmasse der beiden Partner durchschlagen wird (vgl. darüber die Ausführungen Orschanskys) oder anders ausgedrückt, ob die verschiedenen Zeugungsaltersabschnitte eines Menschen auf den Ausgang des Kampfes zwischen den Vererbungstendenzen seiner eigenen Ahnen und derjenigen des Partners einen Einfluß haben? d. h. in moderner, erbbiologischer Ausdrucksweise, ob das Zeugungsalter die Ursache für einen Dominanzwechsel abgeben kann.

Auch die Frage, ob es zu eigenartigen Korrelationsbrüchen führen und ob es unter normalen Verhältnissen latente Anlagen oder Anlagenkomplexe reaktivieren kann, wird einer eingehenden Untersuchung bedürfen.

Vielleicht hängt mit einer Summation von erblichen und Keim-, bzw. Embryonaleinwirkungen (Schwangerschaft) die Behauptung mancher Autoren zusammen (ob sie richtig ist, wäre nachzuprüfen), daß die Belastung durch eine kranke Mutter eine stärkere Belastung der Kinder bedeutet, als wenn sie vom Vater herkommt, und vielleicht in derselben Richtung auch die Erklärung für jene Behauptung (wenn sie richtig ist), daß die Geburt eines Kindes durch eine geisteskranke Mutter eine schädigende Wirkung auf dasselbe bedeutet, welche über die Wirkung reiner Erblichkeit hinausgeht, ja, daß, wie auch geglaubt zu werden scheint, die Austragung des Kindes während der Dauer der mütterlichen Geistesstörung gewissermaßen die Vorbedingung oder doch wenigstens ein starkes prädisponierendes Moment für die spätere gleichnamige oder andersartige Psychose des Kindes ist.

Auch hier sei nochmals, wie auf Seite 548ff. (wo über die Frage diskutiert wird, ob äußere, auf den Keim einwirkende Einflüsse wirkliche Neuerscheinungen zu produzieren vermögen) die ungeheure erbbiologische und rassenhygienische Wichtigkeit der Forderung betont, die erbliche Fixierbarkeit auch dieser ev. milieubedingten Abänderungen festzustellen, unter Berücksichtigung der Sonderfrage, ob die erbliche Fixierung in ihrer Intensität abhängt von der Wiederholung des gleichen Reizes, der die Veränderung beim Elter selbst hervorgerufen hat (vgl. die Versuche Kammerers usw.).

Aus diesen Erwägungen geht hervor, wie ungeheuer wichtig die kritische Lösung der eben besprochenen Spezialfragen für die Degenerations- und Regenerationsmöglichkeiten einer belasteten Familie, also für rassenhygienische Maßnahmen, ist.

Fassen wir mit Rücksicht auf diesen Punkt das Gesagte zusammen, 
so ergibt sich eine dreifache Richtung der Forschung nach Regenerationsund Degenerationsursachen.

Es gilt 1. die Schäden aufzudecken, welche Entartung neu schaffen.

2. Jene Schäden zu eruieren, welche krankhafte Anlagen manifest werden lassen können.

3. Die Erbfolge von Defekten und Krankheitsanlagen festzustellen, welche gesetzmäßig und von äußeren Einflüssen günstiger oder ungünstiger Art fast ganz oder ganz unabhängig vor sich geht und der daher nur durch geschlechtliche Zuchtwahl (Personalauslese) zu begeg. nen ist.

Von den Grundzügen der Gesetzmäßigkeit menschlicher Vererbung und Variabilität abgesehen, lassen sich auf dem Gebiete der Psychiatrie durch das Mittel der Familienforschung noch eine Menge anderer wichtiger Einzelprobleme zur Lösung bringen, die direkt und indirekt wieder die größte Tragweite für die skizzierten Problemstellungen besitzen.

Eine wichtige und verhältnismäßig einfach zu lösende Frage ist die der Fruchtbarkeit der geistig irgendwie Abnormen. Eine solche Fruchtbarkeitsstatistik für die einzelnen psychiatrischen Krankheitsund Defektentitäten fehlt uns noch völlig. Sie muß die Bruttofruchtbarkeit (Gesamtzahl der Kinder bestimmter Individuen) und die Nettofruchtbarkeit (Gesamtzahl der zur Fortpflanzung gelangenden Kinder bestimmter Individuen) betreffen.

Bloße Tatsachen sind hier schon vom allergrößten Werte. Natürlich muß die Statistik sich dann, um Vergleiche mit Nichtgeisteskranken zu ermöglichen, auch ausweisen über alle jene Faktoren, welche, wie wir wissen, die Fruchtbarkeit Nichtgeisteskranker beeinflussen, d. h. über Beruf und Lebenslage, Heiratsfrequenz, Heiratsalter, erreichtes Lebensalter usw.

Dann erst wird man sagen können, ob hier Differenzen bestehen, welche besondere Bedeutung besitzen. Bestehen sie, so wird man ihren eigentlichen Ursachen nachzugehen haben, d. h. eventuellen Einflüssen des Krankheitsbeginns, der Krankheitsdauer, der Krankheitsintensität, der Anstaltsbedürftigkeit, der Sterblichkeit, die mit der Krankheit zusammenhängt, der Internierungsdauer in Anstalten, spezifischer körperlicher, mit der Psychose mehr oder weniger regelmäßig verknüpfter Störungen, welche auf die Kohabitations-, Zeugungs-, Schwangerschafts-, Still- oder Aufzuchtsfähigkeit Einfluß haben.

Viele Einzelfragen tauchen von selbst auf: Wer von uns weiß, wie groß wirklich die Fortpflanzungsrate der von uns als geheilt, gebessert 
und ungebessert Entlassenen sich noch gestaltet? Und wer von uns weiß, wie die Internierung in den Irrenanstalten die Fruchtbarkeit der verschiedenen Krankheitsarten beeinflußt?

Ein Vergleich der Fruchtbarkeit der geistig Defekten in einer gegebenen Familie mit derjenigen der Gesunden derselben Familie wird uns vielfach über Faktum, Mechanismus und Tempo der Entartung und des Aussterbens, sowie der Regeneration und der Vermehrung des ganzen Stammes oder einzelner Zweige desselben Aufklärung verschaffen.

Mit dem Fruchtbarkeitsproblem einerseits, mit der Frage, wer die Utbertragung der Erbanlage übernimmt andererseits, ob der Erkrankte selbst oder in der Hauptsache oder ausschließlich dessen gesunde Geschwister, also mit eminent rassenhygienischen Problemen, hängt die Frage eng zusammen: wieviel Prozent der Geistesstörungen und welche Arten fallen, besonders mit ihrem Beginn, innerhalb die Breite des zeugungsfähigen Alters bzw. jenseits des bevölkerungsdurchschnittlichen Heiratsalters (für die verschiedenen sozialen Klassen verschieden!), wieviel Prozent fallen jenseits der oberen Grenze und welche Arten, und wieviel Prozent und welche Arten fallen vor den Beginn des zeugungsfähigen Alters bzw. vor das durchschnittliche Heiratsalter?

Für die letztere Kategorie ergibt sich, vorausgesetzt, daß es sich um erbliche Anlagestörungen handelt, praktisch die Notwendigkeit, für die anderen Kategorien nur die Möglichkeit, daß die gesunden Geschwister die in Kraft tretenden Erbträger sind.

Für die Kranken der letzteren Kategorie selbst sind die rassenhygienischen Postulate einfach. Die Krankheitsanlage hat sich, wenn man sich so ausdrücken darf, rechtzeitig auffällig gezeigt und ist ein wertvolles zeugungsprophylaktisches Hilfsmittel, selbst in dem Falle, wo nach kürzeren oder längeren Zeiten wieder Heilung und Zeugungsfähigkeit und -gelegenheit eintritt.

Anders mit den psychischen Anlagekrankheiten, die beginnen, nachdem ein Teil oder der ganze Zeugungsabschnitt bereits zurückgelegt ist.

Hier kommt, praktisch, alles darauf-an, die die krankhafte Anlage beherbergende Variante vor dem Erfolg oder Mißerfolg, wie man sich ausdrückt, zu erkennen, $d$. $h$. bevor sie erkrankt und bevor sie Kinder in die Welt setzt (bei vielen manisch-depressiven Kranken, bei Altersmelancholikern, Arteriosklerotikern, Senildementen, Paranoiden, selbst bei manchen Verblödungsprozessen hebephrener und katatonischer Zugehörigkeit).

In diesem Bestreben der Erkennung der Variante vor dem Mißerfolg ist also ein Forschungsziel von höchster Bedeutung gegeben, an dessen Erreichen Kliniker, Psychologen, Anatomen, Stati- 
stiker und Genealogen in gleicher Weise mitzuwirken berufen sind, um so intensiver und dringender, als wir von ihm noch ganz außerordentlich weit entfernt sind zum großen Schaden der Verfeinerung rechtzeitiger zeugungsprognostischer Anhaltspunkte, zum Schaden der Nachkommenschaft und der Probanden selbst, welche zu erkranken bestimmt sind.

Die noch in allen Stücken zu lösende Aufgabe fällt hier wohl in der Hauptsache den Psychiatern zu.

Ich habe von diesem Problem ja bereits im Zusammenhange mit der Korrelationsfrage gesprochen.

Allein noch viel schwieriger und praktisch für die Nachkommenschaft ebenso wichtig ist die Erkennung der Heterozygie eines Individuums, freilich nicht sowohl der dominanten (denn hier dürfte schon das Minimum, was wir durch ihr Aussehen und dessen, was dasselbe für den Ausfall der Nachkommenschaft verspricht, zu rassenhygienisch-prophylaktischen Maßnahmen genügen, siehe Fig. 9, S. 501, Rubr. 3, günstigster Fall einer dominanten heterozygoten Kreuzung) als vielmehr der recessiven Heterozygie, deren Träger ja selbst frei von Abnormität ist, aber sie unter bestimmten Umständen (Partner auch recessiv heterozygot) wieder in der Nachkommenschaft hervortreten läßt (Fig. 10, S. 502, Rubr. 5).

Denn wenn die äußerliche Unterscheidung der gesunden recessiven homozygoten von der gesunden recessiven heterozygoten Variante (in Fig. 10 die äußerliche Unterscheidung der Erbträger mit der Gametenzusammensetzung: Weißer Kreis mit Punkt, also besonders wichtig in Rubrik 3, da hier die Chancen halb und halb stehen) sich selbst in der fernen Zukunft nicht erzielen läßt, wird in diesem Punkte unsere Erbprognose eine bedauerliche Unsicherheit, d. h. die Erkrankungsprognose der Nachkommenschaft eine unbefriedigende ,Nur-Wahrscheinlichkeit“ bewahren, und es wird uns für diese Fälle nur die eine rassenhygienische Remedur der Fremdkreuzung mit anomaliefreiem Blut übrig bleiben, unter Verzichtleistung auf die talent- und tüchtigkeitserhaltenden Wirkungen der Inzucht.

Allein man bedenke, daß wie im Zea-Typus (S. 496) die dominante Heterozygie, also die immanente Spaltungsfähigkeit sehr wohl äußerlich deutlich sein kann, und es liegt prinzipiell, soweit ich sehe, in Analogie zu diesem Falle kein Grund vor, von vornherein die Möglichkeit abzulehnen, daß wenigstens in diesem oder jenem Fall, aber auch gesetzmäßig wie im Fall des Zea-Typus, Träger recessiver heterozygoter Gameten äußerlich unterscheidbar werden können.

Vielleicht gibt sogar ein erfolgreiches, systematisches Studium der korrelativen Verknüpfung der verschiedenen Gametennatur mit verschiedenen anderen, äußerlich wahrnehmbaren Merkmalen 
an lückenlosen Stämmen mit gut charakterisierten Individuen verhältnismäBig bald gewisse Anhaltspunkte in dieser Richtung.

Wichtig und in seiner Bedeutung ganz unaufgeklärt ist die vielbehauptete Tatsache, daß psychische Störungen gerne ante ponieren, d. h. bei Nachkommen in früheren Lebensabschnitten auftreten als bei den Vorfahren, bei jüngeren Geschwistern früher als bei älteren.

Dies wird statistisch noch mehr zu erhärten und festzustellen sein, ob es sich in allen diesen Fällen um gleiche oder ungleiche Störungen handelt, wie viele Generationen dieser anteponierende Prozeß zu umfassen vermag und was er für Fruchtbarkeitsfolgen zeitigt usw.

Kommt auch in regelmäBiger Weise ein Postponieren vor und wo?

Oder läßt sich erweisen, daß diese Phänomene sich restlos durch eine, wenn man so sagen darf, normale Variabilität einer bestimmten Abnormität erklären lassen, welche innerhalb der verschiedenen Generationen auch Abweichungen zum Teil erheblichen Grades in Dauer, Intensität und Verlauf bedingt.

Wie unterscheiden sich Familien mit großer Variabilität innerhalb ihrer Erbkrankheit auch sonst von jenen Familien, wo große Konservativität in der erblichen Überlieferung auch bezüglich der genannten Details herrscht (wo also z. B, auch homochrone Vererbung besteht usw.)?

Wichtige Detailprobleme lassen Betrachtungen vergleichender Psychiatrie innerhalb einer gut bekannten Familie oder eines Familienkomplexes auftauchen, und dies selbst innerhalb Europas, wo es ja große, körperlich und geistig recht verschiedene Rassen und zahlreiche Rassenmischungen gibt.

Man wird dabei, da man ja so viele andere Ursachenfaktoren gleichzeitig registriert, durch entsprechende Ausschließung anderer Ursachen weit sicherer gewisse Eigenarten auf reine Rassenwirkung zurückzuführen vermögen, als wenn man Einzelindividuen von bestimmtem anthropologischen Typus aus dem Familiengefüge herausreißt, um sie miteinander zu vergleichen.

Die wichtigsten Fragen, die es hier zu lösen gibt, sind die, ob die reine Rasse an und für sich zu bestimmten Störungen oder zu bestimmten Ausgestaltungen von Störungen prädisponiert, abgesehen vom Kulturzustande oder der Lebensgewohnheit derselben. $\mathrm{Ob}$ im weiteren Erbgange, nach Zusammentreffen auf eine andere Rasse, die innige Verknüpfung der Störung mit der behafteten Rasse gelöst wird, ob die Abnormität also auch auf die andere Rasse und ihre Mischlinge übergeht und ob dies nach Mendelschen Postulaten geschieht. Welche sonstigen neuen Kombinationen von raßlichen und pathologischen Erbelementen gebildet werden? Kurz wie weit ein Korrelationsbruch 
bei solcher Fremdkreuzung geht und auf welche Eigenschaftskomplexe er sich bezieht.

Ob bei Mischlingen und durch die Mischungen neue Störungen auf dem Gebiet des Zentralnervensystems auftreten können, die beiden Kreuzungsrassen an und für sich fremd sind (Entstehung von Mutationen - stark erblichen Variationen - Atavismen usw. durch Fremdkreuzung).

Daß ein gutes, medizinisch-familiengeschichtliches Material eine vortreffliche Grundlage zu sozialstatistischen Untersuchungen abgeben dürfte, ist selbstverständlich.

Hier kommt besonders in Betracht das Studium der Beziehungen zwischen Familienbeschaffenheit und Militärtauglichkeit, Art der Berufsbefähigung usw.

Die Familienforschung, wie sie hier entwickelt wurde, muß eine systematische, methodische Berufs- und Lebensarbeit, sie darf kein Dilettieren, keine Wappen- und namengenealogische Spielerei, aber auch kein bloßes blinddienendes Anhängsel der Psychiatrie sein. Sie muß sich des Zusammenhanges mit der Experimentalbiologie stets bewußt bleiben. Sie muß sich nach rückwärts erstrecken und in die kollaterale Breite, aber ebensosehr, ja ganz besonders $\mathrm{muß}$ sie in die Zukunft gerichtet sein, damit gerade diejenigen medizinischen Daten unsern Nachfahren beschafft werden, welche wir an den alten zurückliegenden Geschlechtern vermissen.

Die folgenden Generationen müssen daher auf den ihnen von uns überlieferten Traditionen beständig weiter bauen.

Es ist, in seinem höchsten Werte betrachtet, ein Werk, das erst die Zukunft vollenden kann.

Diese Vollendung aber muß reifen, und wir sollten uns daher hüten, voreilig abschließende Urteile zu verkünden, die nach Lage der Dinge in vielen Hauptpunkten nur der Zukunft vorbehalten sein können.

Diese Reife kann wesentlich befördert werden dadurch, daß geeig netes Ma terial, das zeitgenössische Generationen gesammelt haben, an einer oder mehreren Zentralstellen vereinigt wird, um dort die nötige Ordnung und Sichtung und die Verarbeitung zu finden, die es bereits zuläßt.

Ich stelle mir das zunächst so vor, daß sich in einzelnen größeren oder kleineren Ländern wissenschaftliche Zentralen der Familienforschung auf verschiedenen Fachgebieten bilden, die in steter, unmittelbarer Berührung und praktischem Umgang mit Kranken und deren gesunden Verwandten, sowie mit den Behörden des Landes sind, die also in erster Linie über ein eigenes großes, ihnen stets leicht zugängliches Urmaterial verfügen und daher vor Schlüssen am grünen Tisch bewahrt bleiben. 
Unerläßlich ist dabei aber, daß die Fachkollegen die Person ihres Vertrauens, in deren Hand sie die Forschungszentrale gelegt haben, auch mit Rat und Tat unterstützen und umgekehrt Anspruch darauf haben, mit Rat und Tat durch die Zentrale in ihren Bestrebungen gefördert zu werden.

Da die Fachkollegen eines Landes ohnedies durch berufliche und organisatorische Zusammenarbeit enger miteinander verknüpft sind, ließe sich ein Ideen- und Materialaustauch im wesentlichen ohne Herstellung neuer und besonderer Verbindungen leicht bewerkstelligen. Dadurch, daß in der Hauptsache nicht die einzelnen Kollegen, sondern die Zentrale mit der Sichtung und Vervollständigung unter bestimmten Gesichtspunkten betraut würde, fiele ein großer Teil der Arbeitslast auf die Schultern der Zentrale.

Die Behörden müßten nach Aufklärung über die segensreiche Tragweite dieser Forschungsrichtung gebeten werden, in systematischer, fortlaufender Weise mitzuwirken.

Je intensiver die allseitige auch sonstige organische Verbindung der einzelnen Teile des Bezirks sich gestaltet und je intimer die Kenntnis der Umgebung ist, aus der das Material stammt, um so größeren Nutzen wird auch die Familienforschung daraus ziehen, um so zutreffender und kritischer werden die Schlußfolgerungen sein, die man in erb- und variationsbiologischer Hinsicht aus Land und Leuten wird zu ziehen vermögen.

Solche Zentren intimer, eifriger Materialbeschaffung und Sichtung, die für jedes Land einen unermeßlichen Segen bedeuten würden (vgl. auch die Vorschläge von Schüle und H. Roemer) sind die Vorbedingung für das wirkungsvolle Funktionieren einer anderen, dringend notwendigen Einrichtung, deren Schöpfung in letzter Zeit von Alzheimer, v. Gruber, Kraepelin, Sommer in verschiedenen Modifikationen vorgeschlagen wurde, nämlich die Errichtung einer Abteilung für Familienforschung im Reichsgesundheitsamt, deren Hauptaufgabe die Unterstützung der Ursachenforschung wäre, insofern sie nur im Rahmen des Studiums der Familie und des Stammes gelöst werden kann.

Besonders in der Form der Beschränkung der Aufgabe, wie sie Alzheimer dem Reichsgesundheitsamt übertragen möchte, ist der Vorschlag als ein dringendes Bedürfnis zu bezeichnen, nämlich mit besonderen Mitteln und Einrichtungen die Erforschung der Ursachen der Volkskrankheiten zu betreiben, damit diese Ursachen beseitigt und verhütet werden können.

Angesichts des lawinenartigen Anschwellens der finanziellen Lasten, welche dem Staate trotz des beispiellosen Fortschrittes der Bakteriologie usw. aus der Zunahme der Verpflegungsnotwendigkeit Defekter und Kranker aller Art erwachsen, ist die baldige Durchführung unseres 
Vorschlages geradezu ein Gebot der Notwendigkeit, eine staatserhaltende Pflicht.

Als Teil dieser allgemeinen Aufgabe ist die Erforschung psychischer Defekte und Krankheiten zu betrachten, und sie könnte gewiß leicht einem Institut für Familienforschung am Reichsgesundheitsamt übertragen werden, d. h. einer psychiatrisch-genealogischen Sonderabteilung, der die den besonderen Verhältnissen angemessenen Probleme zugewiesen würden, welche aber auch mit verwandten Gebieten Fühlung zu nehmen und sie den allgemeinen Aufgaben des Instituts unterzuordnen hätte.

Dem Menschen ist ein entsetzliches Maß von Krankheit und frühzeitigem Tod beschieden. Es ist aber zurzeit noch unvermeidlich, da wir viele Ursachen nicht kennen und da leider nur durch diesen grausamen Prozeß die Natur die Tüchtigkeit der Familie, des Stammes und der Rasse als solcher einigermaßen aufrecht zu erhalten vermag, indem so im großen und ganzen den Tüchtigen das Feld der Fortpflanzung überlassen wird.

Die Resultate, welche die Untersuchungen nach obigem Plan zeitigen müssen, sollen uns die beherrschbaren Ursachen von Variation und Vererbung, somit von mitwirkenden Ursachen bei Krankheit und frühzeitigem Tod aufdecken und sie meiden lehren nach dem Grundsatz: Wer verhütet, braucht nicht zu heilen!

Die erbarmungslose, mit unsäglichen Schmerzen auf körperlichem und seelischem Gebiet verknüpfte Ausjäte würde so gemildert, auf ein Minimum reduziert.

Das ist der Lohn, der unseren eigenen Anstrengungen und der Mühe unserer Nachfahren werden wird.

Die wichtigsten Problemstellungen sind gegeben. Es ist nur eine Willens- und Geldfrage, ob wir uns ihrer Lösung zuwenden.

\section{Neuere Literatur.}

(Ihr Studium soll dazu dienen, den Eindruck wirklichen, wissenschaftlichen Fortschritts auf dem Gebiete der Vererbung zu vermitteln und den Psychiater dazu führen, die mannigfachen exakten Fragestellungen, welche die Biologie an Hand des Experiments aufgeworfen hat, sich bei der Vererbungs- und Variationsforschung auf psychiatrischem Gebiet stets gegenwärtig zu halten, um auf sie gegebenenfalls ebenso exakte Antworten zu erhalten.

In den aufgeführten Arbeiten aus dem Gebiete der Biologie ist eine reichliche Spezialliteratur zitiert zu finden. Die psychiatrische Literatur betrifft die haupt. sächlichen, in die psychiatrische Vererbungslehre einschlägigen Arbeiten des verflossenen Jahrzehnts.)

\section{Biologie.}

Archiv für Rassen- und Gesellschaftsbiologie, einschließlich Rassenund Gesellschaftshygiene. Jahrgänge 1904, 1905, 1906, 1907, 1908, 
1909, 1910, 1911. Originalien und Referate über sämtliche Fragen der Vererbung, Variabilität, Entartung, Degeneration, Mendel'sche Lehre usw. Verlag von B. G. Teubner, Leipzig.

Bateson, W., Mendels principles of heredity. Cambridge 1909. At the University Press.

- The progress of genetics since the rediscovery of Mendel's papers. In: Progressus Rei Botanicae 1, 1906. Verlag von Gustav Fischer, Jena.

- An address on Mendelian heredity and its application to man. In: Brain 29, 157, 1906 und Brit. med. Journ. 1906.

- Mendelian heredity. 1906. Brain No. 114.

- and Saunders, E. R., Reports to the Evolution Committee of the Royal Society 1. 1902.

- Methoden und Ziele der Vererbungslehre. Biol. Centralbl. 29. 1909.

Ba ur, E., Einige Ergebnisse der experimentellen Vererbungslehre. Med. Klin. (Beiheft) 1908.

- Einführung in die experimentelle Vererbungslehre. Berlin 1911. Gebr. Bornträger.

Beard, Erblichkeit. Brit. med. Journ. No. 2285. 1904.

Beitzke, Vererbung. Berl. klin. Wochenschr. Nr. 36. 1905.

Bevan - Lew is, W., The presidential address on the biological factor in heredity, delivered at the sixty-eighths annual meeting of the Medico-psychological Association held at Wakefield on July $22^{\text {nd }}$ and $23^{d} 1909$. Journ. of mental Science 55, 591-630. 1909.

Biffen, R. H., Studies in the inheritance of disease-resistance, Journ. Agric. Science 2. 1907.

Biometrica, A Journal for the statistical study of biological problems. Cambridge. At the University Press.

Castle, W. E., Heredity of coat characters in guinea pigs and rabbits. Publ. Carnegie Inst. Wash. 1905.

- The heredity of „Angora“" coat in mammals. Sc. N. S. 18. 1903.

- The laws of heredity of Galton and Mendel and some laws governing raceimprovement by selection. Proc. Amer. Acad. Arts and Sc. 39, 223. 1903.

- and Allen, Gl. M., The heredity of Albinism. Contrib. from the zool. Labor. of the Mus. of comp. zool. at Harvard Coll. Cambridge Mass. 1903. Proc. Amer. Ac. 38. 1903.

Chantemesse, Pr., Hérédité. Progrès médical. October 1900, No. 40-42.

Chapeaurouge, A. de, Einiges über Inzucht und ihre Leistungen auf verschiedenen Zuchtgebieten. Hamburg 1909.

Conklin, E. G., The mechanism of heredity. Science 27, 89. 1908.

Correns, C., Gregor Mendels Versuche über Pflanzenhybriden. Bot. Ztg. 58, 230. 1900.

- Experimentelle Untersuchungen über die Entstehung der Arten auf botanischem Gebiet. Archiv f. Rass, u. ges. Biol. 1, 27. 1904.

- Über Vererbungsgesetze. Berlin 1905, bei Gebr. Bornträger.

- Gregor Mendels Briefe an Carl Nägeli 1866-1873. Abt. Math.-Phys. Kgl. Sächs. Ges. d. Wiss. 19. 1905.

- Bastarde zwischen Maisrasse. Biol. Bot. H. 53. 1901.

- Über Bastarde zwischen Rassen von Zea Mays usw. Berl. D. Bot. Ges. 19. 1901.

- Die Ergebnisse der neuesten Bastardforschungen für die Vererbungslehre. Berl. D. Bot: Ges. 19. 1901.

- Der Übergang aus dem homozygotischen in einen heterozygotischen Zustand usw. Berl. D. Bot. Ges. 28. 1910. 
Crzellitzer, A., Sippschafttafeln, ein neues Hilfsmittel zur Erblichkeitsforschung. Med. Reform 1908, Nr. 48, 50 u. 52.

- Methoden der Familienforschung. Zeitschr. f. Ethmol. 1909, 182.

Cuénot, L., Rapport sur l'hérédité. Ass. Franc. Adv. Sc. Congrès de Lyon 1906.

- L'hérédité de la pigmentation chez les souris. Arch. zool. expér. et gén. [4] 2. 1904.

- L'hérédité de la pigmentation chez les souris. 5. Note. Arch. zool. expér. et gén. [4] 6. 1907.

Da maye, L'hérédité collatérale, sa valeur et son importance en pathologie. Revue scientifique 1, 5. série. 1904.

Darbishire, A. D., On the result of crossing Japanese waltzing with albino mice. Biometrica 3. 1904.

- On the result of crossing round with wrinkled peas etc. Proc. Roy. Soc. 80. B. 1908.

- Notes on the results of crossing Japanese waltzing mice etc. Biometrica 2. 1902.

- On the supposed antagonism of Mendelian to biometric theories of heredity. Mem. and Proc. Manchester Lit. and Phil. Soc. 49. 1905.

- On the difference between physiological and statistical Laws of heredity. Mem. and Proc. Manchester Lit. and Phil. Soc, 50. 1906.

- On experimental estimation of the theory of ancestral contributions in heredity. Proc. Roy. Soc. B. 81. 1909.

Daven port, C. B. u. G., Heredity of skin pigment in man. Amer. Naturalist 44, 641. 1910 .

- Heredity of hairform in man. Amer. Naturalist 42, 341-349. 1908.

- Heredity of eye-color in man. Science 26, 589-592, 1907.

- C. B., Inheritance in poultry. Carnegie Publication 52. Washington 1906.

- Degeneration, Albinism and Inbreeding. Science N. S. 28, 44. 1908.

- The imperfection of dominance etc. Amer. Naturalist 44. 1910.

- The origin of black sheep in the flock. Science N. S. 22. 1905.

Dela mare, Hérédité morbide. Journ. de l'anat. et de la phys. No. 6. 1903.

De moll, Zur Lokalisation der Erbanlagen. Zool. Jahrb. 30, 133. 1910. Abt. f. zool. Phys.

Devrient, Ernst, „Familienforschung“ in "Aus Natur und Geisteswelt“ 350. Leipzig 1911. B. G. Teubner.

Dietrich, A., Die Bedeutung der Vererbung für die Pathologie. Tübingen 1902.

Duerst, N. J., Selektion und Pathologie. Hannover 1911. (Arb. d. D. G. f. Züchtungskunde 12.)

Farabee, W. G., Inheritance of digital malformations in man. Papers of the Peabody Museum of American Archaeology and Ethnology Harvard University. Vol. 3, No. 3. Cambridge, Mass., 1905.

Fehlinger, H., Die Gültigkeit der Mendelschen Vererbungsregeln für den Menschen. Polit. Anthrop. Revue 9, 374. 1910.

Fic $k$, R., Vererbungsfragen, Reduktions- und Chromosomen-Hypothesen, Bastardregeln. Ergebn. d. Anat. u. Entwicklungsgesch. 19. 1906.

Fischer, E., Weitere Untersuchungen über die Vererbung erworbener Eigenschaften. Allgem. Zeitschr. f. Entomologie 1902.

Fischer-Planer, Die Vererbung geistiger - Fähigkeiten. Archiv f. system. Philosophie, N. F. 13.1907.

Fruwirth, C., Untersuchungen über den Erfolg und die zweckmäßigste Art der Durchführung von Veredlungs-Auslese-Züchtung bei Pflanzen mit Selbstbefruchtung. Archiv f. Rass.- u. Ges.-Biol, 4, 145. 1907.

- Die Züchtung landwirtschaftlicher Kulturpflanzen. Berlin 1905. 
Galton, F., The average contribution of each several ancestor to the total heritage of the offspring. Proc. Roy. Soc. 61, 401. 1897.

- Natural Inheritance. London 1889.

- Englishmen of Science. London 1874.

- Hereditary genius. London 1892. (Deutsch von Neurath, Leipzig 1910.)

- Entwürfe zu einer Fortpflanzungshygiene. Archiv f. Rass.- u. Ges.-Biol. 1905, S. 812 .

- Correlations and their maesurements. Proc. Roy. Soc. 45, 136. 1888.

Garrod, A. E., The incidence of alkaptonuria, a study, in chemical individuality. Lancet, 13. Dez. 1902.

Goldschmidt, R., Einführung in die Vererbungswissenschaft. Leipzig 1911, Engelmann.

Groß, J., Über einige Beziehungen zwischen Vererbung und Variation. Biol. Centralbl. 26. 1906.

Gruber, M. v., Organisation der Forschung und Sammlung von Materialien über die Entartungsfrage. Concordia 1910.

v. Gruber u. Rüdin, Fortpflanzung, Vererbung, Rassenhygiene. München 1911. J. F. Lehmanns Verlag. Zweite, ergänzte und verbesserte Auflage.

Häcker, Valentin, Allgemeine Vererbungslehre. Braunschweig 1911. Friedrich Viehweg \& Sohn.

— Wandtafeln zur Allg. Biologie. Leipzig 1907 u. 1909. Quelle \& Meyer.

- Über Axolotl-Kreuzungen. II. Mitt. Verh. d. D. Zool. Ges. 1908.

- Über die neueren Ergebnisse der Bastardlehre, ihre zellengeschichtliche Begründung und ihre Bedeutung für die praktische Tierzucht. Archiv f. Rass.u. Ges.-Biol. 1, 321. 1904.

- Bastardierung und Geschlechtszellenbildung. Zool. Jahrb. Suppl. 7. 1904.

Hammer - Stuttgart, Die Bedeutung der Vererbung für die Haut und ihre Erkrankungen. Verhandl. d. Deutsch. Dermatol. Gesellsch. 10. Kongr. Frankfurt a. M., 8.-10. Juni 1908, S. 71-93. Berlin 1908. Julius Springer.

Hansemann, D. v., Deszendenz und Pathologie. Berlin 1909.

Hardy, G. H., Statistical results of Mendelian heredity. Science N. S. 28, 49.

Heide nreich, E., Familiengeschichtliche Quellenkunde. Leipzig 1909. H. A. Ludwig Degener.

Herbst, H., Vererbungsstudien. Archiv f. Entwickl.-Mechanik 21, 22, 24, 27. 1906-1909.

Hertwig, O., Der Kampf um Kernfragen in der Entwicklungs- und Vererbungslehre. Jena 1908.

Hurst, C. C., Experimental studies on heredity in rabbits. Journ. Lin. Soc. 29. 1905.

- Inheritance of eye-colour in man. Proc. Roy. Soc. London. Biometrica 80, 85. 1908.

Johannsen, W., Elemente der exakten Erblichkeitslehre. Jena 1909. Gustav Fischer.

- Über Erblichkeit in Populationen und reinen Linien. Jena 1903.

Kammerer, P., Vererbung erzwungener Fortpflanzungsanpassungen. Archiv f. Entwickl.-Mechanik 25, 7. 1907.

- Beweise für die Vererbung erworbener Eigenschaften. Berlin 1910. (12. Flugschr. d. D. Ges. f. Züchtungskunde.)

Kekule v. Stradonitz, Streifzüge durch die medizinisch-genealogische Literatur. Mitteil. d. Zentralstelle f. deutsche Personen- u. Familiengesch., 3. H. Leipzig 1908.

- Angabe einer Ahnenbezifferungsmethode. „Vierteljahrsschr. f. Wappen-, Siegelu. Familienkunde." 26. Jahrg., S. 64ff. 
Kierman, J. W., Heredity. Medical News, 15. Febr. 1902.

Lang, A., Über Vererbungsversuche. Verh. d. D. Zool. Ges. 1909.

- Über die Mendelschen Gesetze, Art- und Varietätenbildung, Mutation und Variation, insbesondere bei unsern Hain- und Gartenschnecken. Schweiz. Naturf.-Ges. Luzern 1905.

- Über die Bastarde von Helix hortensis Müller und Helix nemoralis L., eine Untersuchung zur experimentellen Vererbungslehre. Jena 1908.

- Über alternative Vererbung bei Hunden. Z. f. indukt. Abstammungslehre 3, 1. 1910.

- Die Erblichkeitsverhältnisse der Ohrenlänge usw. Z. f. indukt. Abstammungslehre 4. 1910 .

Lendenfeld, Robert, Karl Pearsons Untersuchungen über verwandtschaftliche Ahnlichkeit und Vererbung geistiger Eigenschaften. Archiv f. Rass.- u. Ges.-Biologie 1, 78. 1904.

Lenz, Fr., Über die Verbreitung der Lues, speziell in Berlin und ihre Bedeutung als Faltor des Rassentodes. Archiv f. Rass.- u. Ges.-Biol. y, 306. 1910.

Lock, Robert, Recent progress in the study of variation, heredity and evolution. Londion 1907.

Lorenz, Ottokar, Lehrbuch der gesamten wissenschaftlichen Genealogie. Berlin 1898.

Lütgendorf - Leinburg, Familiengeschichte, Stammbaum- und Ahnenprobe. Frankfurt a. M.

Lutz, A., Über einige Stammbäume und die Anwendung der Mendelschen Regel auf die Ophthalmologie (Graefes Archiv 1911).

Martius, F., Krankheitsanlage und Vererbung. Leipzig u. Wien 1905.

- Referat über Krankheitsanlage und Vererbung mit besonderer Berücksichtigung der Tuberkulose. Neurol. Centralbl. 1905, S. 489.

- Das Vererbungsproblem in der Pathologie. Berl. klin. Wochenschr. 1901.

- Das pathologische Vererbungsproblem. Leipzig u. Wien 1909.

- Die Bedeutung der Vererbung für Krankheitsentstehung und Rassenerhaltung. Archiv f. Rass.- u. Ges.-Biol. 7, 470. 1910.

Mendel, Gregor J., Versuche über Pflanzenhybriden. Verh. Naturf.-Verein Brünn 10, 1865 (Ostwalds Klassiker der exakten Wissenschaften).

Morgan, Th. H., Experimentelle Zoologie (deutsch von Rhumbler). Leipzig u. Berlin 1909.

Naegeli-Ackerblom, Die Geminität und ihre erblichen Beziehungen. Virchows Archiv f. pathol. Anat. u. Physiol. 1ro. 1902.

Nettleship, Seven new pedigrees of hereditary cataract. Ophthalmol. Soc. Trans. 29. 1909.

Orschansky, J., Die Vererbung im gesunden und krankhaften Zustande und die Entstehung des Geschlechts beim Menschen. Stuttgart 1903, 347 S.

Orth, J., Die Bedeutung der Erblichkeit für die Pathologie. Berl. klin. Wochenschr. 1906.

- Ererbte und angeborene Krankheiten und die Krankheitsanlagen. In Krankheiten und Ehe (Senator und Kaminer) 1, 26.

Park, Heredity, Scott. med. and surg. Journ. 9, No. 5. 1901.

Pearson, K., Note on inheritance in man. Biometrica 6. 1908.

- The theory of ancestral contributions to heredity.

- On the ancestral gametic correlations of a Mendelian population mating at random. Proc. Roy. Soc. 81, N. 547, B. S. 219 u. 225 . London 1909.

- The law of ancestral inheritance. Biometrica 2, Part. 2. 1903.

- On the Laws of inheritance in Man. I. Inheritance of physical charakter. 
Biometrica 2. 1903. II. On the inheritance of the mental and moral charakters etc. Biometrica 3. 1904.

Pearson, K., Über den Zweck und die Bedeutung einer nationalen Rassenhygiene (NationalEugenik) für den Staat. Archiv f. Rass.- u. Ges.-Biol. 1908, S. 67.

Pick, F., Utber Krankheitsvererbung. In der Deutschen med Wochenschr. 1911.

Plate, L., Über Vererbung und die Notwendigkeit der Gründueg einer Versuchsanstalt für Vererbungs- und Züchtungskunde. Archiv f. Rass.- u. Ges.-Biol. 3, 777. 1906.

- Vererbungslehre und Deszendenztheorie. Antrittsvorlesung zur Übernahme der zoologischen Professur in Jena. Festschr. z. 60. Geburtstag Richard Hertwigs 2, 1910, G. Fischer, Jena.

- Ein Versuch zur Erklärung der gynephoren Vererbung menschlicher Erkrankungen. Archiv f. Rass.- u. Ges.-Biol. 1911, S. 164.

- Über die Bedeutung des Darwinschen Selektionsprinzips und Probleme der Artbildung. 2. A. Leipzig 1903.

Ploetz, A., Ziele und Aufgaben der Rassenhygiene. Deutsche Vierteljahrsschr. f. öffentl. Gesundh. 43, H. 1. S. 164.

- Die Begriffe Rasse und Gesellschaft und einige damit zusammenhängende Probleme. Schriften der Deutschen Gesellsch. f. Soziologie. I. Serie. I. Bd. Tübingen J. C. B. Mohr.

Poll, H., Mischlingsstudien. System und Kreuzung. Sitzungsber. d. Ges. d. Naturf.Fr. Berlin 1907.

Prinzing, Fr., Die Vererbung pathologischer Eigenschaften. Monatsschr. f. Kriminalpsychol. u. Strafrechtsreform 5. 1908.

Punnett, R. C., Mendelism in relation to disease. Proc. Roy. Soc. 1908.

- Mendelismus. Verlag Carl Winiker. Brünn 1910.

Ra y mond, P., L'hérédité morbide. Paris 1905.

Ribbert, H., Die Vererbung der Krankheiten. Pol. anthropol. Revue, Mai 1904.

- Über Vererbung. Vortrag. Marburg, Elwert, 32 S., 1902.

Schuster, E. and Elderton, E. M., The inheritance of ability. London 1907.

Schwalbe, E., Das Problem der Vererbung in der Pathologie. Münch. med. Wochenschr. 1903, Nr. 37, S. 1579; Nr. 38, S. 1460.

- Mißbildung und Variationslehre. Jena 1910.

Se mon, R., Der Stand der Frage nach der Vererbung erworbener Eigenschaften. Fortschr. d. naturw. Forschung 2, I. 1910.

Sommer, Max, Die Brown-Séquardsche Meerschweinchenepilepsie und ihre Übertragung auf die Nachkommen. Inaug.-Diss. Jena 1900 u. Zieglers Beitr. z. path. Anat. u. z. allg. Pathol. 2\%. 1900.

- Zur Frage der erblichen Übertragbarkeit der Brown-Séquardschen Meerschweinchenepilepsie. Neurol. Centralbl. 1901, S. 152.

- R., Goethes Wetzlarer Verwandtschaft. Leipzig 1908.

- Goethe im Lichte der Vererbungslehre. Leipzig 1908.

StandfuB, M., Zur Frage der Gestaltung und Vererbung auf Grund 28jähriger Experimente. Leipzig 1902.

- Die Resultate 30 jähriger Experimente. Verh.d. Schweiz. Naturf.-Ges. Luzern 1905.

Steiger, A., Studien über die erblichen Verhältnisse der Hornhautkrümmung. Zeitschr. f. Augenheilk. 16, 229. 1906.

Stockmann, Heredity. Glasgow med. Journ. 65, No. 3.

Stras burger, E., Die stofflichen Grundlagen der organischen Vererbung. Jena 1905.

The Treasury of human inheritance (thesaurus hereditatis). University of London. Francis Galton Laboratory for National Eugenics. Mit Abbildungen und Stammbäumen. London, Dulau and Co. 
Thomson, J. A., Heredity. London 1908.

Tschermak, E. v., Der moderne Stand des Vererbungsproblems. Archiv f. Rass.u. Ges.-Biol. 5, 305.1908.

- Die Mendelsche Lehre und die Galtonsche Theorie vom Ahnenerbe. Archiv f. Rass.- u. Ges.-Biol. 2, 663.1905.

Vries, H. de, Die Mutationstheorie. Leipzig 1901.

Weinberg, W., Pathologische Vererbung und genealogische Statistik. Archiv f. klin. Med. 88.1903.

- Über den Nachweis der Vererbung beim Menschen. Jahreshefte d. württ. Vereins f. vaterl. Naturkunde 1908.

- Über Vererbungsgesetze beim Menschen. Zeitschr. f. indulstive Abstammungsu. Vererbungslehre 1, 2. 1909.

- Aufgabe und Methode der Familienstatistik bei medizinisch-biologischen Problemen. Zeitschr. f. soz. Med. 3. 1907.

Weismann, A., Semons „Mneme" und die Vererbung erworbener Eigenschaften. Archiv f. Rass.- u. Ges.-Biol. 3, 1. 1906.

Weldon, W. F. R., Inheritance in animals and plants. In: Lectures on the method of Science. Oxford 1906.

- Albinism in Sicily and Mendels Law. Biometrica 3, 107. 1904.

Zeitschrift für induktive Abstammungs- und Vererbungslehre.

Ziegler, H. E., Vererbungslehre in der Biologie. Jena 1905.

Ziermer, M., Genealogische Studien über die Vererbung geistiger Eigenschaften (Konstanz der Familiencharaktere im Mannesstamme), nachgewiesen an einem Material von 1334 Waldauer Haushaltungen. Archiv. f. Rass.- u. Ges.Biol. 5, 178, 327. 1908.

\section{Psychiatrie.}

Alzheimer, A., Ist die Einrichtung einer psychiatrischen Abteilung im Reichsgesundheitsamt erstrebenswert? Zeitschr. f. d. ges. Neurol. u. Psych. Orig. 6, 242. 1911 .

Ameline, De l'hérédité, et en particulier de l'hérédité similaire dans la paralysie générale. Annales méd.-psychol. Mai u. Juni 1900.

Anton, G., Alkoholismus und Erblichkeit. Psych. Wochenschr. 1901. Nr. 14. Apert, E., Maladies familiales et maladies congénitales. Paris 1907, 364 S.

Arna ud, F. S., Les maladies mentales familiales. Revue de psychiatrie, n. s. III. Paris 1900.

Benedikt, Degenerationskrankheiten des Nervensystems. Deutsche med. Wochenschr. Nr. 25. 1906.

Berze, J., Die hereditären Beziehungen der Dementia praecox. Leipzig-Wien 1910.

- Die manisch-depressive Familie H. Beitrag zur Hereditätslehre. Monatsschr. f. Psych. 26, 270. 1909.

Bing, R., Die heredofamiliären Degenerationen des Nervensystems in erblichkeitstheoretischer, allgemeinpathologischer, rassenbiologischer Beziehung. Med. Klin. 1906.

- Heredofamiliäre Nervenkrankheiten. Deutsches Archiv f. klin. Medizin 83, H. 3 u. 4. 1905.

Bisch off, E., Über familiäre Geisteskrankheiten. Jahrb. f. Psych. 1905, S. 109. Blin, Contribution à l'étude de l'hérédité dans les états mentaux de l'enfant. Congr. internat. de méd. C. r. Paris 1900, sect. de psych. 522.

Bolton, Josef Shaw., Evidence as to the existence of heredity of insanity and of parental and family degeneracy in the subjects of dementia paralytica. Journ. of mental Science 54, 26. 1908. 
Bourneville, Note statistique sur le rôle de la consanguinité dans l'étiologie de l'épilepsie, de l'hystérie, de l'idiotie et de l'imbécillité. Progr. Med., 4. Mai 1901.

Brachet, A., Pathologie mentale des rois de France, Louis XI et ses ascendants. Paris 1903.

Bratz, Über Vererbung. Vortrag im psychiatrischen Verein zu Berlin, Sitzung vom 18. Dezember 1909. Mit Diskussion. Neurol. Centralbl. 1910, Nr. 2.

Burnet, Anne, A study of heredity among the women in the State Hospital at Clarinda, Jowa. Womans M. J. Toledo 9, 375-380. 1900.

Buschan, G., Bedeutung der Verwandtschaftsheiraten für die Nachkommenschaft. Neuland des Wissens 1. 1910.

Cannon and Rosanoff, Preliminary report of a study of heredity in insanity in the light of the Mendelian laws. Journ. of nervous and ment. disease 38, 272. 1911.

Cox, W. H., Degeneratie, eene copulativogene correlatiestoornis. Psychiatrische en Neurologische Bladen 1907, No. 1, 77 S. $8^{\circ}, 6$ Tafeln. Ref. im Archiv f. Rass.- u. Ges.-Biol. 1908, S. 125.

Dana, Charles F., The modern views of heredity with the study of a frequently inherited psychosis. Medical Record ry, 345. 1910.

Die m, Otto, Die psycho-neurotische erbliche Belastung der Geistesgesunden und der Geisteskranken. Archiv f. Rass.- u. Ges.-Biol. 2, 215-252 u.336-368. 1905.

Doran, A consideration of the hereditary factors in Epilepsy. Amer. Journ. of Insanity 60. 1903.

Elmiger, Beiträge zum Irresein bei Zwillingen. Psych.-Neurol. Wochenschr. 1910/11, Nr. 8, S. 78; Nr. 9, S. 85.

Er makow, J., Observations personelles sur les psychoses alcooliques et l'hérédité alcoolique chez les battants de la guerre russo-japonnaise. Arch. intern. do neurol. 1909; ref. Neurol. Centralbl. 1910, S. 1357.

Etchepare, B., Folie familiale, délire d'interprétation antilogique communiqué entre sept personnes. Annales méd.-psychol. 68, 5. 1910.

Fa user, Endogene Symptomenkomplexe bei exogenen Krankheitsformen. Allgem. Zeitschr. f. Psych. 62, 165.

Fitschen, Eleonore. Die Beziehung der Heredität zum periodischen Irresein. Monatsschr. f. Psych. \%, 127, 224. Berlin 1900.

Fitzgerald, F. G., A study of phases of family psychosis. Bull. of the Ontario Hospitals for the Insane 2, 23.1908.

Foerster, Über die klinischen Formen der Psychosen bei direkter Erblichkeit. Mit Diskussion. Allgem. Zeitschr. f. Psych. 64, H. 1, S. 176. 1907.

Fornaca, Sull influenza dell' ereditarietà morbosa nella paralisi progressiva. Rivista sperim. di freniatria 33, Fascic. II, III. 1907.

Fouque, P., Maladies mentales familiales. Paris 1900.

Frankhauser, K., Geschwisterpsychosen. Zeitschr. f. d. ges. Neurol. u. Psych. Orig. 5, 52. 1911.

Fuller, L. O., Alcoholism, crime and insanity. Journ. of mental Science 55, 692. 1909. Aetiology (heredity), S. 700.

Galip pe, V., L'hérédité des stigmates de dégénérescence et les familles souveraines. Paris 1905, Masson et Cie. et Gaz. des hôp. 1905, S. 903.

- Hérédité similaire. Revue de méd., No. 11 et 12.1901.

Goddard, Heredity of feeble-mindedness. Amer. Breeders Magazine 1, Nr. 3, S. 165-178. 1910. Washington D. C.

Goring, C., On the inheritance of the diathesis of phthisis and insanity; a statistical study, based upon the family history of 1,500 Criminals. Drapers 
Company Research memoirs, Departement of Applied Mathematics. University College, London; published by Dulau and Co. 3 shill. 1909.

Gottgetreu, Die Bedeutung der Vererbung bei der Dementia paralytica. Inaug.Diss. Jena 1902.

Gowers, Heredity in diseases of the nervous system. Brit. med. Journ. 1908.

Graeter, Dementia praecox mit Alcoholismus chronicus. Ätiologie, S. 178 u. f. Leipzig 1909.

Hähnle, E., Der heutige Stand der Erblichkeitsfrage in der Neuropsychopathologie. Neurol. Centralbl. 1904, Nr. 18, S. 843-853 u. 882-892.

Hartmann, Über die hereditären Verhältnisse bei Verbrechern. Monatsschr. $f$. Kriminalpsychol. 1904, 1. Jahrg., S. 493.

Hartzell, W. H., The burden of hereditary mental diseases. Lehigh Valley M. Mag., Easton, Pa. 12, 21-27. 1901.

Her mann, Paralytikerkinder. Münch. med. Wochenschr. 1909, Nr. 20, S. 1025 bis 1027.

Heron, David, A first study of the statistics of insanity and the inheritance of the insane diathesis. No. 2 der Eugenics Laboratory Memoirs. Aus dem Francis Galton Laboratory for National Eugenics. London 1907. Dulau and Co., $33 \mathrm{~S}$.

Hirschl, Jos. Adolph, Zur Ätiologie der Dementia praecox. Vortrag, gehalten im Verein f. Psych. u. Neurol. in Wien am 12. März 1907. Jahrb. f. Psych.

Hoche, A., Zur Frage der erblichen Belastung bei Geisteskrankheiten. Med. Klin. 1905, Nr. 18, S. 427.

- Kultur und Geisteskrankheit. Freiburg i. Br. 1910.

Jakowenko, W., Erblichkeit bei Geisteskrankheit. 1904, H. 1/2 des Journ. neuropathol. i psich. (Organ der Neuropathol. Gesellsch. zu Moskau). 1905, S. 683. Ref. in Centralbl. f. Neurol. u. Psych. 1905, S. 683.

Jeffrey, G. R., The significance of Heredity and the Neuro-Insane Constitution as important factors in the Production of mental disease. Journ. of ment. Sc. April 1910. Vol. 56, 273-296.

Jendrassik, Ernst, Beiträge zur Kenntnis der hereditären Krankheiten. Deutsche Zeitschr. f. Nervenheilk. 22, H. 5 u. 6. 1902.

- Die hereditären Krankheiten. Lewandowskis Handb. d. Neurol. 2, 321. Berlin 1911.

Joerger, J., Die Familie Zero. Archiv f. Rass.- u. Ges.-Biol. 2, 494. 1905.

Jones, W. H. S., Ross, R. und Ellett, G. G., Malaria, a neglected fact in the history of Greece and Rome. Cambridge 1907.

Kalmus, Untersuchungen über erbliche Belastung. 6. Jahresversamml. Norddeutsch. Psychiater u. Neurologen. Centralbl. f. Nervenheilk. u. Psych. 1905 , S. 75.

Karpas und Morris, J., Contribution to our knowledge of the aetiology of Dementia praecox. New York Med. Journ., 5. u. 12. Dez. 1908.

Karplus, J. P., Zur Kenntnis der Variabilität und Vererbung am Zentralnervensystem des Menschen und einiger Säugetiere. Leipzig u. Wien 1907.

Kekule v. Stradonitz, Die Untersuchung von Vererbungsfragen und die Degeneration der spanischen Habsburger. Archiv f. Psych. 35, H. 3. 1902.

Kirchhoff, Fragen aus dem Gebiete der Erblichkeit. Allgem. Zeitschr. f. Psych. 56,6, S. 871.

Knapp, Philip Coombs, Heredity in diseases of the nervous system with especial reference to heredity in epilepsy. Read at the meeting of the Amer. Neurol. Assoc. at Washington, 8. Mai 1907. 
Kneidl, C. (Prag), Beitrag zur Statistik und Atiologie der Epilepsie. (Casopis čes. lék. Prag 1904.) Ref. Neurol. Centralbl. 1905, S. 220.

Koch, J. L. A., Die erbliche Belastung bei den Psychopathien. Kinderfehler, 8. Jahrg., 1903, H. I.

König, W., The problem of heredity from the psychiatrical aspect. Brit. med. Journ., Oct. 15th, No. 2285.

Kraepelin, Emil, Zur Entartungsfrage. Centralbl. f. Nervenheilk. u. Psych. 1908, 2. Oktoberheft.

Krauss, W. C., Heredity with a study of the statistics of the New York State hospitals. Buffalo M. J. 1901-02 n. s. 41, 892-899.

Krauss, Kennenburg, Über Vererbung von Geisteskrankheiten. 23. Versamml. d. südwestdeutsch. Irrenärzte in Stuttgart am 1. u. 2. Sept. 1902. Allgem. Zeitschr. f. Psych. 1903, S. 224.

Kreichgauer, Rosa., Zur Frage der Vererbung von Geisteskrankheiten. Inaug.Diss. Freiburg i. Br. 1909 u. Centralbl. f. Neurol. u. Psych. 1909, S. 877-885.

Lagriffe, L., Recherches sur l'hérédité dans les maladies mentales. Archives d'anthropolog. criminelle et méd. légale 1910.

Lange, Fr., Middelfart, Dänemark, Degeneration in Families: Observations in a lunatic Asylum. Engl. Übersetzung. London 1907. $8^{\circ}$. 3 shill. 6 d. net.

- Slaegter, Jagttagelser fra en Sindsygeanstalt. Kopenhagen 1904, 146 S., $3 \mathrm{Kr}$.

Levi-Branchini, Marco, Sull' età di comparsa e sull' influenza dell' ereditarietà nella patogenesi della demenza primitiva o precoce. Rivista sperim. di freniatria 29, Fasc. 3.

Lewis, W., Alcoholism, crime and insanity. Journ. of mental Science 52, 203. 1906. Heredity S. 212.

Liepmann, Psychose der Mutter und Psychose der Tochter, mit Krankenvorstellung. Sitzung d. Psychiatr. Verein zu Berlin vom 24. Juni 1905. Allgem. Zeitschr. f. Psych. 1905, S. 841.

Lomer, Weibliche Trinker und ihre Nachkommenschaft. Pol. Anthr. Rev. 1907.

Lui, A., Eredità ed alcoolismo. Annali di Nevrologia 18. Napoli 1900.

Lundborg, Hermann, Über Geschlechterforschungs- und Erblichkeitshygiene. Allgem. Zeitschr. f. Psych. 1906, S. 175.

— Om släktforskning och ärftlighets hygien. Upsala Läkaref. förhandl. 1905. Ref. in Centralbl. f. Nervenheilk. u. Psych. 5, 660. 1906.

Mac Donald, P. W., Medico-Psychological Association of Great Britain and Ireland Presidential Address delivered July 25th, 1907 by Mac Donald. Journ. of mental Science 1907, S. 677. Heredity, S. 695.

- C. F., The etiological potency of heredity in mental diseases. Philad. M. J. 8, 739-741. 1901 .

Me Gugan, A., The importance of heredity as a cause of insanity. Journ. of the Amer. med. Assoo. Chicago 38, 371-376. 1902.

- Heredity and the investigation of insanity. Amer. Med. Philad. 2, 166. 1901.

Marandon de Montyel, Prédisposition et causes directes en étiologie mentale. Revue d. Psych. 1905, S. 115.

Marc, Über familiäres Auftreten der progressiven Paralyse. Allgem. Zeitschr. f. Psych. 61, 5, 660, 1904.

Marioni, Contributions à l'étude de l'hérédité chez les paralytiques généraux. Thèse 1900.

Me ndel, G., Geisteskrankheiten und Ehe. In: Krankh. u. Ehe (Senator u. Kaminer), München 1904, S. 642. 
Merzbacher, L., Gesetzmäßigkeiten in der Vererbung und Verbreitung verschiedener hereditär-familiärer Erkrankungen (mit 19 Fig. u. 2 farb. Tafeln). Archiv f. Rass.- u. Ges.-Biol. 1909, S. 172-198.

Meyer, Die Ursachen der Geisteskrankheiten. Jena 1907.

Moebius, P. J., Über Entartung. Wiesbaden 1909, Bergmann.

Mondio, G., Eredità e degenerazione nello sviluppo della demenza consecutiva ed in quello delle stereotipie riscontrate in essa. Merita la demenza consecutiva un capitolo a sè in nosografia mentale? Rivista mensile di Neuropat. e Psich. 1900, No. 4-5.

Mönkemöller, Eine Vagabundenfamilie. Arch. f. Kriminalanthropol. 1907.

Mott, F. W., The hereditary aspects of nervous and mental diseases (Huxley Lecture). The Lancet, Oktober 8, 1910, S. 1057-1064.

Müller, Her m., Einige Beziehungen des Alkoholismus zur Ätiologie der Epilepsie. Monatssčhr. f. Psych. u. Neurol. 28, Erg.-Heft, S. 1. 1910.

Munson, J. F., The role of heredity and other factors in the production of traumatic epilepsy. Epilepsia Vol. 2, 343.

Naecke, P., Erblichkeit und Prädisposition resp. Degeneration bei der progressiven Paralyse der Irren. Archiv f. Psych. 41, 295-366. 1906.

- Die Rolle der erblichen Belastung bei der progressiven Paralyse der Irren. Neurol. Centralbl. 1900, Nr. 16.

- Das prozentual ausgedrückte Heiratsrisiko bez. Ausbruchs und Vererbung von Geistes- und Nervenkrankheiten. Allgem. Zeitschr. f. Psych. 63, 3. u. 4. H., S. $482-505.1906$.

Naegeli - Ackerblom, Medikohistorischer Beitrag zur Frage der erblichen Belastung. Therap. Monatsschr., H. 6. 1907.

Neisser, Cle m., Individualität und Psychose. Vortrag bei der Naturforscherversammlung 1905 .

Nina-Rodrigues, Atavisme psychique et paranoia. Arch. d' anthrop. crim. Lyon u. Paris 18, 325-355. 1902.

Obersteiner, Heinr., Zur Frage der hereditären Übertragbarkeit akquirierter pathologischer Zustände. Neurol. Centralbl. 1900, Nr. 11, S. 498.

Pearson, Karl, On the inheritance of the mental and moral charakters in man, and its comparison with the inheritance of the physical charakters. Journal of the Antropological Institute of Great Britain 33, 179-237. 1903.

- Inheritance of want of mental balance. Brit. med. Journ. 1905 (I).

Pei pers, Consanguinität in der Ehe und deren Folgen für die Deszendenz. Allgem. Zeitschr. f. Psych. 58, H. 5, S. 793-862. 1901.

Pelletin, M., L'hérédité biologique et l'hérédité psychologique. La Medecine moderne 1905.

Petró, F., Della pazzia analogo o gemellare. Annali di freniatria e sc. affini 20 , 142. 1910.

Pieraccini, Ulteriore contributo allo studio delle leggi che regolano la ereditarietà psicopatica. Rivista sperim. di freniatria 28, 326--330. Reggio-Emilia 1902.

Pilcz, Alexander, Beitrag zur Lehre der Heredität. Festschr. z. Feier d. 25jähr. Bestandes d. Neurol. Institutes a. d. Wiener Universität, herausg. v. Dr. Otto Marburg, I. Teil, 1, 282-309. Leipzig u. Wien 1907.

Pla ut und Göring, Untersuchungen an Kindern und Ehegatten von Paralytikern. Münch. med. Wochenschr. 1911, S. 1959.

Rehm, P., Die Ergebnisse der Untersuchung von Kindern manisch-depressiver Kranker. Zeitschr. f. d. Erforsch. u. Behandl. d. jugendl. Schwachsinns 3. 1909.

Reid, C. A., Alkoholism, a study in heredity. London 1901. 
Reid, C. A., Principles of heredity with some applications. London 1905.

Reiss, Eduard, Konstitutionelle Verstimmung und manisch-depressives Irresein. Berlin, J. Springer, 1910. Vererbung, S. 255-261.

Ris, F., Vorstellung von Familiengruppen Geisteskranker. Korrespondenzbl. f. Schweiz Ärzte 32, 447-449. Basel 1902.

Römer, L. S. A. M., Beiträge zur Erkenntnis des Uranismus. Heft 1: Die uranische Familie, Untersuchungen über die Aszendenz der Uranier. Leipzig, Amsterdam 1906. $107 \mathrm{~S}$.

- Die erbliche Belastung des Zentralnervensystems bei Uraniern, geistig gesunden Menschen und Geisteskranken. Jahrb. f. sexuelle Zwischenstufen, 7. Jahrg., 1, 67. Leipzig 1905.

Roemer, Hans, Zur Symptomatologie und Genealogie der psychischen Epilepsie in der epileptischen Anlage. Allgem. Zeitschr. f. Psych. 68, 588-627. 1910.

- Eine Stammliste aller amtlich bekannt werdenden Fälle von Geisteskrankheit, Psychiatr. Neurol. Wochenschr. 13. Jahrg. 1911. H. 10, S. 94.

Rogue de Fursac, J., L'hérédité dans l'avarice. Journ. de Psychol. norm. et Pathol. Mai-Juni 1909.

Rohde, Das Vererbungsproblem in der Neuro- und Psychopathologie. 79. Versamml. Deutsch. Naturf. u. Ärzte in Dresden vom 15.-21. Sept. 1907. Neurol. Centralbl. 1907, S. 972.

Roubinowitsch et Phulpin, Contribution à l'étiologie du syndrome de la démence précoce. Annales méd.-psychol. Juni 1903.

Roubinowitsch, J., et Levaditi, Le rôle de la syphilis dans l'étiologie de la démence précoce. Gazette des Hôp. Juni 1909.

Rüdi n, Ergänzende Bemerkungen zu Otto Diems Artikel: ,,Die psychoneurotische, erbliche Belastung der Geistesgesunden und der Geisteskranken." Archiv f. Rass.- u. Ges.-Biol. 2, 470. 1905.

R ybakow, Th., Alkoholismus und Erblichkeit. Monatsschr. f. Psych. u. Neurol. 20, Erg.-Heft, S. $221-234.1906$.

Sandy, C. William, Studies in heredity with examples. Amer. Journ. of Insanity 66, 587--589. 1910.

Sanger - Brow n, Heredity and epilepsy. The alienist and neurologist. July 1901.

Schlöss, Zur Kenntnis der Ätiologie der angebornen und frühzeitig erworbenen psychischen Defektzustände. Psych.-Neurol. Wochenschr. Nr. 48-50. 1907.

Schlub, H. O., Ủber Geisteskrankheit bei Geschwistern. Allgem. Zeitschr. f. Psych. 66, 3. u. 4. H., S. 514-541. 1909.

Schüle, Über die Frage des Heiratens von früher Geisteskranken. Leipzig 1904, S. Hirzel.

- Über die Frage des Heiratens von früher Geisteskranken. II. (Geisteskrankheit und Ehe.) Anlegung der statistischen Tabellen über Erblichkeit. Klinische und biologische Fragestellungen. Genealogische Stammbäume von $20 \mathrm{zykli}$ schen Geisteskranken (mit Tabelle). Vorschläge zur Prophylaxe. Mit 11 Tafeln. Berlin 1905. G. Reimer. $46 \mathrm{~S}$.

- Nochmals das Heiraten von früher Geisteskranken. Ref. Psych.-Neurol. Wochenschr. 1904, Nr. 100.

- Vorlage eines Bogens für Feststellung der Erblichkeit. Jahresversamml. d. Deutsch. Vereins f. Psychiatrie in Dresden am 28. u. 29. April 1905. Neurol. Centralbl. 1905, S. 484.

Schwarzwald, Bernard, De la démence précoce familiale. Thèse de Doctorat. Lausanne. Imprimerie Cosmopolite. Pré-du-Marché 45. 1907.

Semelaigne, René, Family mental diseases. Journ. of mental Science 1901, S. 150. 
Sichel, Max, Über die Geistesstörungen bei den Juden. Neurol. Centralbl. 1908, Nr. 8, S. 351.

- Der Alkohol als Ursache der Belastung. Neurol. Centralbl. 29, 738-748. 1910.

$\mathrm{S} k$ lare k und van Vle u ten, Gleichzeitige geistige Erkrankung bei 3 Geschwistern. Allgem. Zeitschr. f. Psych. 61, 5.

Sommer, R., Psychiatrische Untersuchung eines Falles von Mord und Selbstmord mit Studien über Familiengeschichte und Erblichkeit. Sommers Klinik f. psych. u. nervöse Krankh. 1, 1, S. 55 .

- Die Beziehungen zwischen Psychologie, Psychopathologie und Kriminalpsychologie vom Standpunkte der Vererbungslehre. Vortrag, gehalten in der Gesellsch. f. soz. Medizin usw. am 21. Februar 1909 in Berlin, nebst Diskussion. Bibl. f. soz. Medizin usw., H. 3. Berlin 1910. AAllgem. Med. Verl.Anstalt. $30 \mathrm{~S}$.

- Zur Theorie der Verwandtenehen und des Ahnenverlustes bei Menschen und Tieren 5, 4. H. Halle a. S. 1910.

- Familienforschung und Vererbungslehre. Leipzig 1907.

- Bemerkungen zu einem Fall von ererbter Sechsfingerigkeit 5, 4. H. Halle a. S. 1910.

- Psychiatrie und Familienforschung. Allgem. Zeitschr. f. Psych. 1907, H. 2-3, S. 463 .

Stansfield, T. E. K., Heredity and Insanity. Journ. of. ment. Se. January 1911.

Stelzner, H. F., Die psychopathische Konstitution und ihre soziologische Bedeutung. Berlin 1911. S. Karger. Ätiologische Momente, S. 13-74.

Strohmayer, W., Über die Bedeutung der Individualstatistik bei der Erblichkeitsfrage in der Neuro- und Psychopathologie. Münch. med. Wochenschr. 48, 1786, 1842. 1901 .

- Die Ahnentafel der Könige Ludwig II. und Otto I. von Bayern. Ein genealogisch-psychiatrischer Deutungsversuch. Archiv f. Rass.- u. Ges.-Biol. 1910 , S. 65.

- Über den Wert genealogischer Betrachtungsweise in der psychiatrischen Erblichkeitslehre. Monatsschr. f. Psych. u. Neurol. 22, Erg.-Heft, S. 11 õ. 1907. (Festschrift für Otto Binswanger.)

- Zur Kritik der Feststellung und der Bewertung psychoneurotischer erblicher Belastung. Archiv f. Rass.- u. Ges.-Biol. 5, 478. 1908.

Sutherland, J. F., Recidivism. Part. II. Journ. of mental Science 1907, S. 568. Heredity, S. 584.

- Recidivism. Part. III. Journ. of mental Science 1908, S. 68. Biometrics and National Eugenics, S. 71. Degeneracy, S. 72. Causation of Rec., S. 73.

Thompson, W. N., Heredity and the transmission of the insane diathesis. Vermont M. Month. Burlington 6, 325-337. 1900. Auch: Journ. Med. n. Sc. Portland \%, 337-342. 1900/01.

Tigges, W., Die Abnormitäten der Aszendenz in Beziehung zur Deszendenz. Allgem. Zeitschr. f. Psych. 1907, S. 891.

- Die Gefährdung der Nachkommenschaft durch Psychosen, Neurosen und verwandte Zustände der Aszendenz. Allg. Zeitschr. f. Psych. 63, 448-481. 1906.

- Untersuchungen über die erblich belasteten Geisteskranken. Allgem. Zeitschr. f. Psych. 64, H. 1, S. 1. 1907. 47 S.

Tiling, Th., Individuelle Geistesartung und Geistesstörung. Wiesbaden 1904.

- Zur Ätiologie der Geistesstörungen. Centralbl. f. Nervenheilk. u. Psych., Sept. 1903, S. 561.

Tredgold, A. F., Remarks on the subsequent history of children born whilst the mother was insance. Lancet 1, 1380-1385. London 1902. 
Trénel, Maladies mentales familiales. Annales méd.-psychol. Paris 1900, 8. s., 11, $96-107$.

Urquhart, The Morison lectures. On insanity, with special reference to heredity and prognosis. Journ. of mental Science 1907, April, Nr. 221, S. 233.

Vogt, R., Om Arvelighed. Separataftryk af Tidsskrift for Nordisk Retsmedicin og Psykiatri.

Vorster, Über die Vererbung endogener Psychosen. Monatsschr. f. Psychiatrie 9. 1901.

- Zur erblichen Übertragung der Geisteskrankheiten. Diskussion. Allgem. Zeitschr. f. Psych. 1901, S. 493.

VoB, Über die Bedeutung der Entartung in der Psychiatrie und Neurologie. In: VerhandI. d. Med. Vereins zu Greifswald, Jahrg. 1909, S. 44, Leipzig, Georg Thieme, 1910. Und in: „Deutsche med. Wochenschr." 1909-10.

Wagner v. Ja uregg, Über erbliche Belastung. Antrittsvorlesung. Wiener klin. Wochenschr. 1902, Nr. 44.

- Einiges über erbliche Belastung. Wiener klin. Wochenschr. 1906, Nr. 1.

Weinberg, W., Verwandtenehe und Geisteskrankheit. Archiv f. Rass.- u. Ges.Biol. 1907, S. $471-475$.

- Statistik und Vererbung in der Psychiatrie. Klinik f. psych. u. nervöse Krankh. 5, H. 1. 1910.

Wiglesworth, J., The Presidential Address delivered at the 61. Annual meeting of the Medico-Psychological Association, held at Liverpool on July 24 th., 1902. Heredity and Insanity. Journ. of mental Science 1902, S. $611-645$.

Wille, Über erbliche Übertragung von Geisteskrankheiten. Korrespondenzbl. f. Schweiz. Ärzte 1902, S. 603.

Wlassak, R., Das Problem der Degeneration. Vortrag, gehalten im Akadem. Abstinenzverein Wien. Aus: Internat. Monatsschr. z. Erforsch. d. Alkoholism. u. Bekämpf. d. Trinksitten. 20. Jahrg., H. 3. März 1910.

Wolfsohn, R yssia, Die Heredität bei Dementia praecox. Allgem. Zeitschr. f. Psych. 64, $347 \mathrm{ff}$. 1907.

Woods, Frederick Ada ms, Mental and moral heredity in royalty. A statistical study of history and sociology. New York 1906, $12^{\circ}$, VIII, 312 s. ill.

Word, J. M. S. und Urquhart, A. R., A Family tree illustrative of insanity and suicide. Journ. of mental Science 1901, Oktober, S. 764. 